\title{
Impact of Structure Modification on Cardiomyocyte Functionality
}

\author{
Dissertation \\ FOR THE AWARD OF THE DEGREE \\ "Doctor rerum naturalium" \\ of the University of Göttingen
}

within the doctoral program

Göttingen Graduate Center for Neurosciences, Biophysics, and Molecular Biosciences (GGNB), Physics of Biological and Complex Systems (PBCS)

\author{
by \\ Filippo G. Cosi \\ from \\ Bagno a Ripoli (FI), Italy
}

Göttingen, 2020 


\section{Thesis Committee}

Apl. Prof. Dr. Ulrich Parlitz,

Biomedical Physics Group,

Max Planck Institute for Dynamics and Self-Organization

Prof. Dr. Stefan Klumpe,

Institute for the Dynamics of Complex Systems,

Georg-August-Universität Göttingen

Dr. Andreas Neef,

Center for Biostructural Imaging of Neurodegeneration, Max-Planck-Institute for Experimental Medicine

\section{Members of the Examination Board}

Referee: APl. Prof. Dr. Ulrich Parlitz,

Biomedical Physics Group,

Max Planck Institute for Dynamics and Self-Organization

$2^{\text {nd }}$ Referee: Prof. Dr. Stefan Klumpe, Institute for the Dynamics of Complex Systems,

Georg-August-Universität Göttingen

Further members of the Examination Board:

Dr. Andreas NeEF,

Center for Biostructural Imaging of Neurodegeneration, Max-Planck-Institute for Experimental Medicine

Dr. DAVID ZWICKER,

Theory of Biological Fluids,

Max Planck Institute for Dynamics and Self-Organization

Prof. Dr. Andreas Janshoff,

Institute for Physical Chemistry,

Georg-August-Universität Göttingen

Prof. Dr. Peter Sollich,

Institute for Theoretical Physics,

Georg-August-Universität Göttingen

Date of oral examination: 2020-02-27 
"With four parameters I can fit an elephant, and with five I can make him wiggle his trunk."

John von Neumann

"Everything should be made as simple as possible, but no simpler."

Albert Einstein

"All models are wrong; some models are useful."

George E. P. Box 



\begin{abstract}
Cardiac diseases are often related to defects in subcellular components of the heart's main constituents, the heart muscle cells also called cardiac myocytes. These biological cells periodically contract due to excitation-contraction coupling, i.e. an interplay of intracellular ion dynamics and membrane potential which is centered around calcium release units (CRUs). Especially alterations of the functions and the geometry of CRUs may lead to distorted intracellular ion and voltage dynamics resulting in a malfunctioning cell.

While the functions of CRUs are well studied, the knowledge about their geometry is still incomplete. However, recently the ryanodine receptors 2 (RyRs), i.e. calcium handling channels in CRUs, have been found to form elongated clusters rather than being densely packed into lattice-like configurations, as was previously assumed. This experimental observation represents a good reason to investigate the influence of the geometrical arrangement of ionic channels on the dynamics of cardiomyocytes.

In this thesis a multiscale mathematical model is employed to quantify the impact different RyR arrangements in CRUs have on the ion dynamics and voltage dynamics of cardiac myocytes. The model describes the microscopic and stochastic processes of calcium release as well as the intracellular mesoscopic ion diffusion and action potential dynamics. Using this model we show that not only the shape of the RyR cluster, but also the density and the arrangement of the channels are found to be relevant for the cell dynamics. The numerical simulations proved changes in the microscopic structure and geometry of cell components to significantly affect observed quantities like the action potential duration or the average peak calcium concentration and thus the whole cardiomyocyte functionality.

Moreover, since the employed mathematical model is computationally expensive, a method for the generation and validation of a cheaper numerical model is applied. Using this approach a meta model is generated based on the results from only a few hundred simulation runs of the complex original model. Computationally faster regressions based on the meta model can thus now accompany the multiscale mathematical model improving the efficiency, with which descriptive and relevant predictions can be made.
\end{abstract}




\section{Table of contents}

Glossary and Abbreviations viii

1 Introduction $\quad 1$

1.1 The Inner Universe of Cardiomyocytes . . . . . . . . . . . . . . . . . 2

1.1.1 Cardiomyocytes in Experimental Studies . . . . . . . . . . . . 2

1.1.2 Mathematical Models of Cardiomyocytes . . . . . . . . . . . . 3

1.2 Scope of this Thesis . . . . . . . . . . . . . . . . . 4

1.2.1 Physiological Insights into Subcellular Geometry . . . . . . . . . 4

1.2.2 Does Geometry Play a Major Role? . . . . . . . . . . . . . . 5

1.2 .3 Surrogate Model . . . . . . . . . . . . . . . . . . 6

1.3 Structure of the Thesis . . . . . . . . . . . . . . . 8

2 Mathematical Model and Methods $\quad 11$

2.1 Modelling Calcium release in Cardiomyocytes . . . . . . . . . . . . . . 11

2.1.1 Calcium Diffusion in Cardiomyocytes . . . . . . . . . . . . . 12

2.1.2 Calcium Release Unit . . . . . . . . . . . . . . . . . . . . 14

2.1.3 Channel Placements . . . . . . . . . . . . . . . . . . 14

2.2 Cleftdyn: Multiscale Model of Intracellular Calcium Cycling . . . . . . 16

2.2.1 Model Geometry . . . . . . . . . . . . . . . . 17

2.2 .2 Electrophysiology . . . . . . . . . . . . . . . . . . 20

2.2 .3 Ion Diffusion . . . . . . . . . . . . . . . . . . . . 21

2.2.4 Cleft Dynamics . . . . . . . . . . . . . . . . . 23

2.2.5 Remarks on Computational Costs . . . . . . . . . . . . . 27

3 Parameter Analysis 29

3.1 Generating Model Populations . . . . . . . . . . . . . . . . . 29

3.1 .1 Simulation Types . . . . . . . . . . . . . . . . 30

3.1 .2 Biomarkers . . . . . . . . . . . . . . . . 31

3.1 .3 Parameter Input . . . . . . . . . . . . . . . . . . . . . 33

3.1.4 Sampling Action Potentials and Sparks . . . . . . . . . . . . . . 34

3.2 Parameter and Biomarker Estimation . . . . . . . . . . . . . . . 36

3.2.1 Linear Sensitivity Analysis and Sobol Coefficients . . . . . . . . 37

3.2 .2 Biomarker Sieve . . . . . . . . . . . . . . . . 39

3.3 Polynomial Chaos Expansion . . . . . . . . . . . . . . . . . 41

3.3.1 Polynomial Chaos Expansion - Theory . . . . . . . . . . . . 41 
3.3.2 Polynomial Chaos Expansion - Application . . . . . . . . . . . . 42

3.3.3 Contours of a Response Function . . . . . . . . . . . . . . . . . 44

3.4 Impact of Structure Modifications . . . . . . . . . . . . . . . . . . . 45

3.4.1 Regular and Soeller Placement . . . . . . . . . . . . . . . . 45

3.4.2 Different Placement Measures . . . . . . . . . . . . . . . 45

3.4.3 Convex Hull and Occupancy . . . . . . . . . . . . . . . . . 48

4 Impact of Structure Modifications on Cardiomyocyte Functionality $\mathbf{5 1}$

4.1 Dyadic Structure-Function Relation in Cardiomyocytes . . . . . . . . . 51

4.2 Polynomial Chaos Expansion - Revisited . . . . . . . . . . . . . 63

4.2.1 Surrogate Model . . . . . . . . . . . . . . . . . . . . . 64

4.2.2 On the variable nature of spark simulations . . . . . . . . . 69

5 Discussion and Conclusions $\quad 71$

5.1 Impact of Geometry on Global Cardiac Myocyte Functionality . . . . . 71

5.2 Construction of a Surrogate Model . . . . . . . . . . . . . . 72

5.3 Outlook ............................... 73

5.4 Conclusion . . . . . . . . . . . . . . . . . . . . . 74

$\begin{array}{ll}\text { References } & 75\end{array}$

$\begin{array}{ll}\text { Appendix A Appendix } & 87\end{array}$ 


\title{
Glossary and Abbreviations
}

\author{
Abbreviations \\ AP Action potential \\ APD Action potential duration. $\mathrm{APD}_{X}$ at level $X$. \\ CICR Calcium-induced calcium release \\ CM Cardiomyocyte, heart muscle cell \\ CPU Central processing unit \\ CRU Calcium release unit \\ CVS Cross validation score
}

DUNE Distributed and unified numerics environment

ECC Excitation-contraction coupling

FDHM Full duration at half maximum

FEM Finite element method

j/nSR Junctional/network sarcoplasmic reticulum

LASSO Least absolute shrinkage and selection operator

LCC L-type calcium channel, sometimes also DHC

MPI Message passing interface

NCX Sodium calcium exchanger

ODE Ordinary differential equation

PCE Polynomial chaos expansion

PCL Pacing cycle length

PDE Partial differential equation

RyR Ryanodine receptor 2

viii 
SERCA Sarco/endoplasmic reticulum $\mathrm{Ca}^{2+}$-ATPase

SR Sarcoplasmic reticulum

\section{Glossary}

action potential Excitation of the membrane voltage in biological cells, here in cardiac muscle cells.

biomarker Analysed output of a mathematical model that represents a characteristic cell function or behaviour.

cardiomyocyte Also cardiac myocyte, single heart muscle cell. It is the main component of which the heart is made. It allows the organ to beat via periodic contractions.

clfetdyn Name of the multiscale mathematical model of cardiomyocytes used in this work and developed in the Falcke lab of the Max Delbrück Center for Molecular Medicine in Berlin.

cytosol Inner gel-like substance inside biological cells.

organelle Subcellular component that resides in the cytosol and with specific functions for the cell.

quark Reduced calcium release from one single CRU, in which one or only few RyR open at the same time.

sarcolemma Membrane that surrounds biological cells and thus encloses the cytosol.

Soeller/Jayasinghe placement Arrangement of RyR channels in CRUs according to the algorithm developed in [1].

spark Event of calcium release from one single CRU, in which the majority of RyRs opens simultaneously.

surrogate/meta model Numerical model that mimics the behaviour of a computational expensive and complex mathematical model.

Z-disc Transversal cut through a cardiomyocyte, on which LCCs and RyR cluster are colocalised. Also known as Z-line.

\section{Symbols}

$\alpha \quad$ Penalisation factor of the LASSO regression method

$g_{\text {LCC }} \quad$ LCC channel permeability

$g_{\mathrm{RyR}} \quad$ RyR channel permeability

$k_{\text {close }}$ Closing rate of RyR channels

$k_{\text {plus }} \quad$ Opening rate of RyR channels

$V p_{\max }$ Maximal strength of SERCA pump uptake 



\section{Chapter 1}

\section{Introduction}

The heart is the organ responsible for the vital blood delivery to lungs and body. Its main components are heart muscle cells, commonly referred to as cardiac myocytes or cardiomyocytes. Several types of heart diseases and heart failure are often attributable to defects in the structure and functioning of cardiomyocytes [2-5], which have been the focus of many studies over the past years as reported in [6].

Experimental investigations are the foundations of a deeper understanding of the functioning of cardiac cells. With the insight gained in experiments techniques for treating diseased cardiomyocytes can be developed and further studied. These types of studies can be both challenging and invasive [1,2,7]. The challenges are often linked to technical limitations that allow only for limited resolutions, while some preparation and analysis techniques of the cardiomyocytes can alter their states possibly causing cellular components to malfunction [8-10].

In this regard mathematical models often proved to be helpful in accompanying experimental observations on the path to new insight [11], since they are capable of resolving spatial and temporal regions that otherwise would remain undisclosed. Mathematical models are always a simplified description of reality, they thus need to keep up with new insights in order to be reliable.

A recent experimental study on the structure of subcellular components [1] found how the geometry differs from the shapes that where previously assumed in mathematical models as e.g. in [12]. In the work of this thesis I improved an existing mathematical model by including these novel physiological insights about cardiomyocytes and studying its relevance for cardiac cell modelling. Further I show how a method for the construction of reliable alternative and computationally faster models (compared to an original complex one) can be applied. 


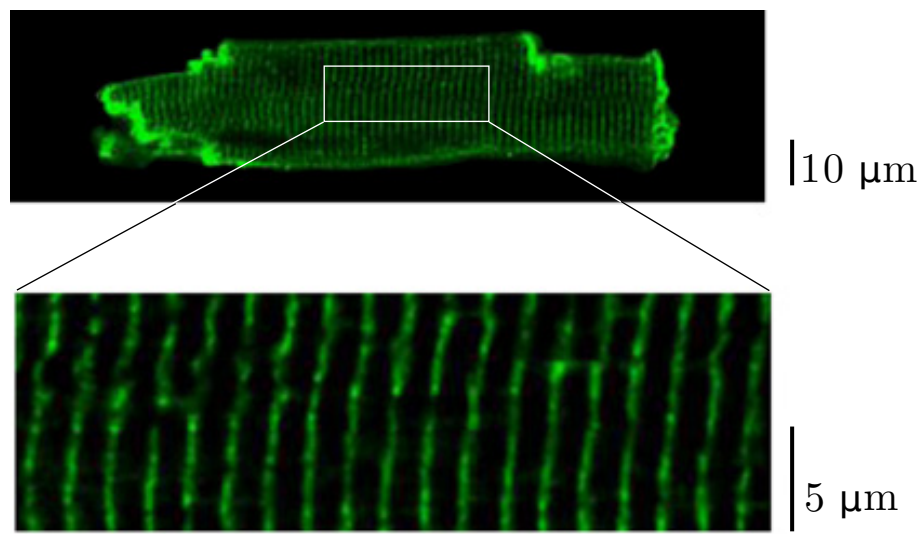

Figure 1.1 - Confocal image of a single cardiomyocyte, upper panel, with an enlargement of the boxed region, lower panel. Cellular membrane and so-called Z-lines are made visible via a fluorescent dye (di-8-ANNEPS) as green vertical stripes. Adapted from [13] with permissions.

\subsection{The Inner Universe of Cardiomyocytes}

\subsubsection{Cardiomyocytes in Experimental Studies}

Figure 1.1 shows a confocal image of a cardiomyocyte with an enlargement of the selected area [13]. Well visible are the so-called Z-lines in the cell interior, where the centres of calcium handling, the calcium release units (short CRUs) are localised. Calcium is essential in muscle cells for allowing a proper contraction of the muscle fibres [14].

Understanding the calcium dynamics in myocytes helps to shine light on the mechanism that enables contraction of the cells. Cardiomyocytes with altered or malfunctioning calcium dynamics thus cease to contract properly [2]. Although such malfunctions are harmless for the heart when they affect few isolated cells, in cases where large regions of the heart tissue are affected it can threaten the proper contraction of the organ.

In order to study the intracellular calcium dynamics it is necessary to gain knowledge on the subcellular components that are responsible for the calcium handling. Therefore, in section 2.1 a detailed description of the geometrical arrangement of subcellular organelles and their functions in cardiomyocytes will be provided.

In recent years experimental techniques to visualise intracellular structures in cardiomyocytes are improving with a fast pace: from imaging single ion channels and channel clusters $[1,7,15,16]$ to resolving the organelle structures inside the cell $[9,10$, $17]$ to studies done on entire cells $[2,18]$. There are many experimental investigations on cardiac cells that allow for an understanding of the mechanisms that permit a healthy functioning of the myocyte. Often diseased cardiac cells are studied by altering the function of ion channels of the cardiomyocyte using specific drugs $[2,5,19,20]$. A remarkable study on how drugs acting on a channel level can affect the functions of 
the entire heart was done on atrial cardiomyocytes by Lou et al. [21].

Many of the experimental studies cited above are affected by some technical limitations, particularly by the resolution of microscopy techniques while imaging living cells $[1,7,10,22]$. Here mathematical models are capable of resolving the ion dynamics in undisclosed subcellular regions. Further a mathematical model can function as a "cheap" drug test, when the relations between subcellular components are well known and a link between experiment and computational model can be established. Experimental insight can thus be accompanied and enriched by mathematical models that are capable of mimicking the function of both healthy and diseased subcellular components. The following section is a summary and characterisation of some of the models that are used for studying cardiomyocytes from a computational point of view.

\subsubsection{Mathematical Models of Cardiomyocytes}

The number of mathematical models mimicking the intracellular ion and specifically calcium dynamics and its link to the membrane voltage in the context of cardiac myocytes is ever-growing. From resolving ion channels and their surroundings $[12$, 23-28] through spatially detailed models [29-35] up to ionic descriptions of the cellular dynamics [36, 37] the universe of intracellular cardiac mathematical modelling is extended and rich. The goal is often the same, i.e. to be an aid for experiments indicating possible pathways for further investigations and to better understand the behaviour of cardiomyocytes when perturbed from their usual regime, e.g. due to diseases.

The interest in finding the roots and implications of cellular diseases that might threaten the proper functioning of the heart muscle is strong $[2,4,5,20,34,38,39]$. Because of their relative cheap costs once they are established, i.e. the equations are set and the numerics implemented, mathematical models can be used to gain knowledge on phenomena that, at the current state of the art, cannot be studied experimentally. This insight can be both trendsetting for new studies and reduce the number of costly and some times invasive experiments to be performed. A requirement for mathematical models in this regard is the proximity to experimental studies, which would be the accuracy with which they describe nature and thus with which they can be compared to experimental results.

Mathematical models studying the inner functions of cardiomyocytes can be categorised into three main groups, concerning the dimensions that are considered: models as $[12,27,28]$ have their focus on calcium diffusion in and around single CRUs, models as $[29,32,34,39,40]$ neglect the detailed description of calcium diffusion in CRUs, but resolve the entire cell and its ion dynamics, finally pure ionic models as [36, 37, 41, 42] set their focus, as their naming already suggests, on ionic currents and membrane potential dynamics neglecting any spatial discretisation inside the cell. The models of the third category are often integrated in those of the second to describe the ion currents and the membrane potential [29, 33, 38, 39].

One mathematical model deserves particular attention, since it cannot be categorised as the others, it rather tries to cover all of them. This model was developed by the 
Martin Falcke lab of the Max Delbrück Center for Molecular Medicine in Berlin [23, $24,35,43-47]$ and describes a very broad spectrum of spatial-temporal dimensions. This characteristic is not present, to my knowledge, in any other mathematical model describing intra-cardiomyocyte calcium dynamics. The multiscale nature of the model allows for a range that starts from the spatial and temporal resolution of single ion channels on one side and reaches the description of calcium diffusion and membrane potential dynamics on the other. Therefore this multiscale model closes a gap between microscopic channel gating (very well described in [27] and [12]) and the ion diffusion in the macroscopic cell interior, as described in e.g. [29]. With this model it is possible to gain insight on the effects microscopic modifications of the subcellular structure have on the function of the cardiomyocyte on a cellular and thus macroscopic level.

Considering these points as crucial for the development of mathematical models in the field of cardiomyocyte research, I chose to adopt this model for the present study.

\subsection{Scope of this Thesis}

\subsubsection{Physiological Insights into Subcellular Geometry}

Recent experimental findings on the structure of subcellular components in cardiomyocytes [1], particularly a new insight on the shape of components of CRUs, motivated me to include this new knowledge in the mathematical model by Martin Falcke, which in the following I will refer to as cleftdyn. In [1] the shape and density of clusters of Ryanodine Receptors 2 (particular calcium channels, short RyR) is revealed. These clusters represent one of the keystones of calcium ion dynamics and thus contraction inside muscle cells. In the lack of deeper knowledge, many studies model RyR clusters as being formed by channels placed on a lattice-like grid [27] often in the shape of a rectangle or square $[12,35]$; In many cases the rectangular grid is further spatially represented by a point source $[29,33,48]$. In some cases the density of the RyRs inside the cluster is reduced [13].

Jayasinghe, Clowsley and coauthors used newly developed microscopy techniques (which are described in detail in reference [22]) and found nature not to reflect this arrangement of RyR channels, but rather to have ion channels distributed in elongated clusters and with a lower density [1]. In figure 1.2 two sketches of the RyR arrangement are shown: in figure 1.2a as it was assumed to be before the new experimental study and in figure $1.2 \mathrm{~b}$ as it actually was observed [1].

The difference is remarkable, especially the packing of RyR channels between the outline-based view and the molecular resolution view changes dramatically. In the former the RyR cluster is simply filled with channels, while in the latter the RyRs are positioned with gaps between neighbouring channels. The authors of [1] further developed an algorithm that mimics the arrangement of RyRs with high agreement with what was observed in experiments and is best described as a "random walk" of the channel placement. 


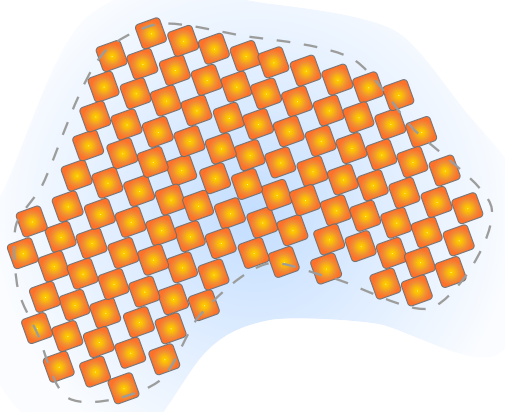

(a) - Outline-based view of RyR clusters as it is assumed to be when standard microscopy methods are employed.

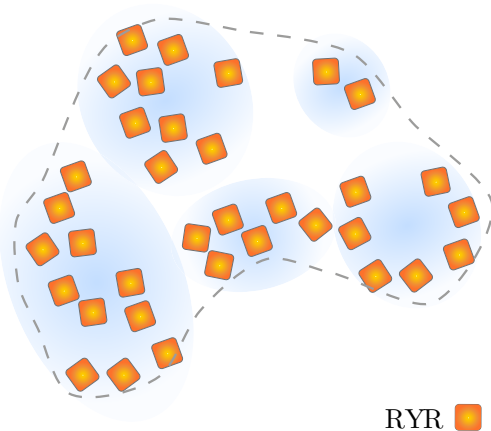

(b) - Molecular resolution view with molecules visualised as they are observed with the DNA-Paint microscopy technique.

Figure 1.2 - Sketches of cluster arrangements of the same RyR cluster for two different microscopy techniques. RyRs are depicted as orange squares and the cluster outlines as grey dashed lines. The figures were adapted from [1], Creative Commons, CC BY 4.0.

These findings are valuable insights for the community studying cardiomyocytes, since such a resolution of RyR clusters was not achieved before; the authors thus ask for mathematical models "to be refined to capture the effect of such gaps on excitability using the data provided here" [1].

An inclusion of the algorithm for channel placement developed by Jayasinghe, Clowsley and coauthors, which is best described as a random walk for the channel placement [1], into cleftdyn, considering the description capabilities of the mathematical model, appears to be obvious.

\subsubsection{Does Geometry Play a Major Role?}

The question whether modifications in the microscopic structure in cardiomyocytes affect the dynamics on a whole cell level is relevant for a proper mathematical modelling of intracellular processes (see for instance the differences between [35] and [33]), but also for the understanding of diseases such as the loss of calcium channels as it happens during so called t-tubule disruption $[13,49]$.

In [35] the authors employed cleftdyn to demonstrate how changes of the dynamics ruling the CRU, i.e. the microscopic actors of calcium handling, would affect the ion concentrations and the electrophysiology of the entire cardiomyocyte. As outlined in section 1.2.1, the distribution of RyRs inside CRUs was assumed to be lattice-like and the RyRs were considered to form densely packed channel clusters [12, 27, 35]. The inclusion of new placement methods like [1] into the mathematical model would represent a microscopical structure modification, whose impact on the cardiomyocyte is unknown. In this work I undertook, together with my coauthors Martin Falcke and Wolfgang Giese, the endeavour of understanding how much variations in the microscopical geometry would affect the functioning of cardiomyocytes on a broader scale [50]. 
The choice of the "right" microscopic dynamics is often a central discussion point in studies on cardiomyocytes, especially ionic models as [37] and whole cell models that approximate the CRU as a point source as e.g. [29, 48]. On the other hand the choice of the CRU structure plays a role merely with spatially limited models, where only one or few units are present as in [27] and [12]. With the role of a gap closer, cleftdyn is thus perfectly suited to be the mathematical model capable of investigating the impact different structures of the subcellular calcium release unit have on cellular level.

We can now pose a scientific relevant question: does the microscopic geometry of the subcellular CRUs play a major role in the global ion handling and electrophysiology of cardiomyocytes?

Our hypothesis is that the arrangement of channels in subcellular components does influence the whole cell ion and membrane potential dynamics and in this thesis I describe an approach for confirming this hypothesis.

It is interesting to note how previous studies made with cleftdyn did focus on functional properties of the CRU $[24,45]$ and their effect on the cell dynamics [35], while now we approach the structure of the subcellular components and study their impact on the cellular level.

The incorporation into cleftdyn of the placement algorithm developed in [1] represented a major change in the structure of the model and raised the question of how strong it would impact on the models output, asking for a robustness check of the model itself. The rework that had to be done on cleftdyn motivated me and my coauthors to employ statistical techniques only recently applied to cardiac studies [51-53] for the assessment of our model's robustness.

\subsubsection{Surrogate Model}

Solving cardiac models numerically can be computationally demanding allowing only for a reduced number of simulation runs. In general these high computational costs are correlated with the model complexity and capability of resolving as many details as possible. Cleftdyn is certainly such a demanding model (see section 2.2.5 for details), from which a statistical relevant study in a classical sense, i.e. a Monte Carlo like generation of thousands of simulation runs [54], cannot be performed.

The aid of so-called surrogate models (often also called emulators or meta models) can be a support for computational demanding models. An example of a successfully created surrogate model that is accompanied by a complex and detail-rich mathematical model is given by [55]. The label surrogate is inspired by the terminology found in [52] and [56], where a computationally faster model is created starting from the outcome of a demanding one.

In figure 1.3 a schematic representation of how a surrogate model works is shown. The orange boxes on the left represent the input and the complex mathematical model, while the blue boxes on the right represent the output. This is often divided into raw output, which for example could be the time evolution of the membrane potential, and the analysed output, which in the example case could be represented by the mean value of the action potential duration as function of some model parameter. The green box 
depicts the surrogate model that is created on the basis of the analysed output and which then becomes a "shortcut" between the initial model's input and the analysed data.

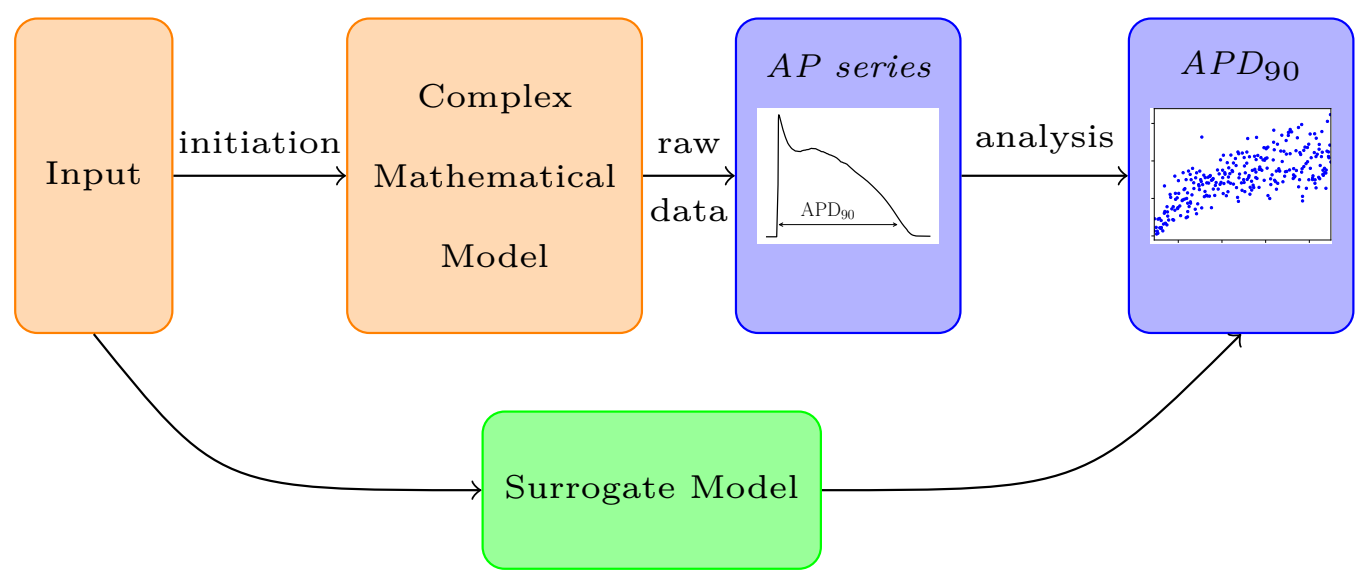

Figure 1.3 - Schematic representation of a general workflow for mathematical models showing the "shortcut" taken by a surrogate model. In orange the input and the main mathematical model are shown, in blue the output is shown. The left output box represents the raw output as for instance the time evolution of the membrane potential, the right one represents the analysed data as e.g. the action potential duration. A surrogate or meta model, depicted here as the green box, is able to map the input to the analysed output avoiding a long computation time.

The idea behind surrogate models is to represent an approximation of the main computationally demanding model and to allow for many and/or long simulation runs in cases where the original model could only produce a reduced number of them. Attention has to be paid here, since the surrogate model does not replace the original one, it is rather a supplement that allows for finer scans of the parameter space and/or further prediction.

Creating a surrogate model can be undertaken in many ways [53]. Since this was not the main focus of our study, the creation of a meta model was not part of the initial goals, but during estimation and tuning of the model's input parameters it turned out to be an endeavour that would open new possibilities to cleftdyn. As mentioned we needed to calibrate input parameters of the modified mathematical model to allow for physiological output. Since the number of simulations that can be performed with cleftdyn is small (compared to e.g. a Monte Carlo like simulation that produces thousands of repetitions), we had to employ methods from statistics and uncertainty quantification [57] to properly analyse the model's outputs.

In the specific case we did use a method introduced by Norbert Wiener, the polynomial chaos expansion (PCE) [58] to perform regressions on a multidimensional parameter space that is sparsely filled. Polynomial chaos expansion uses orthonormal polynomials to fit a function to a high dimensional data set, of which the input parameters are varied [56]. This regression allowed us to create a function that would 
represent a sort of look up table of the original outputs of cleftdyn, with the possibility to explore further parameter combinations that were not part of the original simulations.

This regression represents a surrogate model, which we used first to asses the parameter ranges that would deliver physiological output of cleftdyn. This surrogate model can be further used to gain insight on regions in parameter space barely accessible in their entirety by cleftdyn, in other terms it allows for a "shortcut" as the one sketched in figure 1.3.

Since cleftdyn represents a mathematical model that covers a wide range of spatial scales from the resolution of single ion channels [23] up to whole cell dynamics [35], a method that allows the construction of a surrogate model based on cleftdyn is a valuable tool that enhances the possibilities of analysis.

\subsection{Structure of the Thesis}

This thesis is structured as follows:

- Chapter 2 is divided into two main sections:

- Section 2.1 describes the main components and functions of cardiomyocytes with a focus on the literature describing the intracellular structures and processes. Special attention is paid to the motivation of this project, which is detailed in section 2.1.3, where the newly developed placement method for RyRs is described [1].

- In the second section 2.2 the multiscale mathematical model is outlined in its principal components together with the equations that describe the intracellular processes.

- In chapter 3 the methods of the data analysis together with the main results are discussed.

- Sections 3.1 and 3.2 describe which output measures where chosen and how they where handled and analysed in order to calibrate the input parameters of the model. The first steps towards the assessment of model parameters yielding output that can be considered to be physiological are described here.

- In section 3.3 the theory behind polynomial chaos expansion is outlined together with its application to the output measures described in the previous two main sections.

- Section 3.4 discusses the impact that the inclusion of the new channel placement method [1] has on the cellular dynamics in the frame of cleftdyn.

- Chapter 4 consists of two main sections:

- Section 4.1 contains the publication [50], in which the results outlined in chapter 3 are presented with further details. 
- Section 4.2 holds an analysis and a discussion of the generation of the surrogate model using polynomial chaos expansion based on the results of [50]. In this section I also describe an optimiser for the regression method, that allows for the generation of a robust meta model.

- In chapter 5 I summarise the results of the present study discussing its main and most relevant aspects. Finally I sketch a pathway for further investigations. 



\section{Chapter 2}

\section{Mathematical Model and Methods}

This chapter focuses on the considered structures and cell components. Here I describe the main functions and elements of the modelled heart muscle cells, the cardiomyocytes. Further I describe the mathematical model employed to carry out the present study embedding it in the current state of the art considering the advances and achievements of the recent years (see section 2.1.3). The chapter is divided into two main sections that consider physiological functioning of cardiomyocytes in connection to mathematical models on one and the implementation of one specific mathematical model, the one used in this thesis, on the other hand.

\subsection{Modelling Calcium Release in Cardiomyocytes}

Muscle cells (myocytes) are responsible for the mechanical contraction and relaxation of organs. The following description is visualised by a sketch in figure 2.1, where all the main components of muscle cells are depicted [60].

Myocytes are mainly composed of muscle fibres so-called myofibrils, in which the interplay between actin and myosin allows the cell to contract. One of the main actors in this process is calcium, which binds to a molecule known as troponin $C$ and then to actin initiating contraction [14, 61-63].

Calcium is stored in an organelle that surrounds the myofibrils, the sarcoplasmic reticulum (SR), which is often divided into network and junctional SR (the latter is labelled terminal cisternae in figure 2.1). This subdivision allows to distinguish between those sections of the SR that are simply calcium reservoirs and those that are connected to the cell exterior [64]. This connection is made possible by so-called t-tubules, which are invaginations of the sarcolemma (the cell membrane) towards the interior of the cell. In figure 2.1 these connecting points are described as triads, while in this study we used the terms calcium release unit (short CRU), dyadic cleft or simply cleft to address the main component responsible for the calcium handling in the cell.

The placement of t-tubules is not random in the cell, but is characterised by colocalisation along transversal sections of it. These regions have a higher density of CRUs than the rest of the cell and are therefore of special interest when studying 


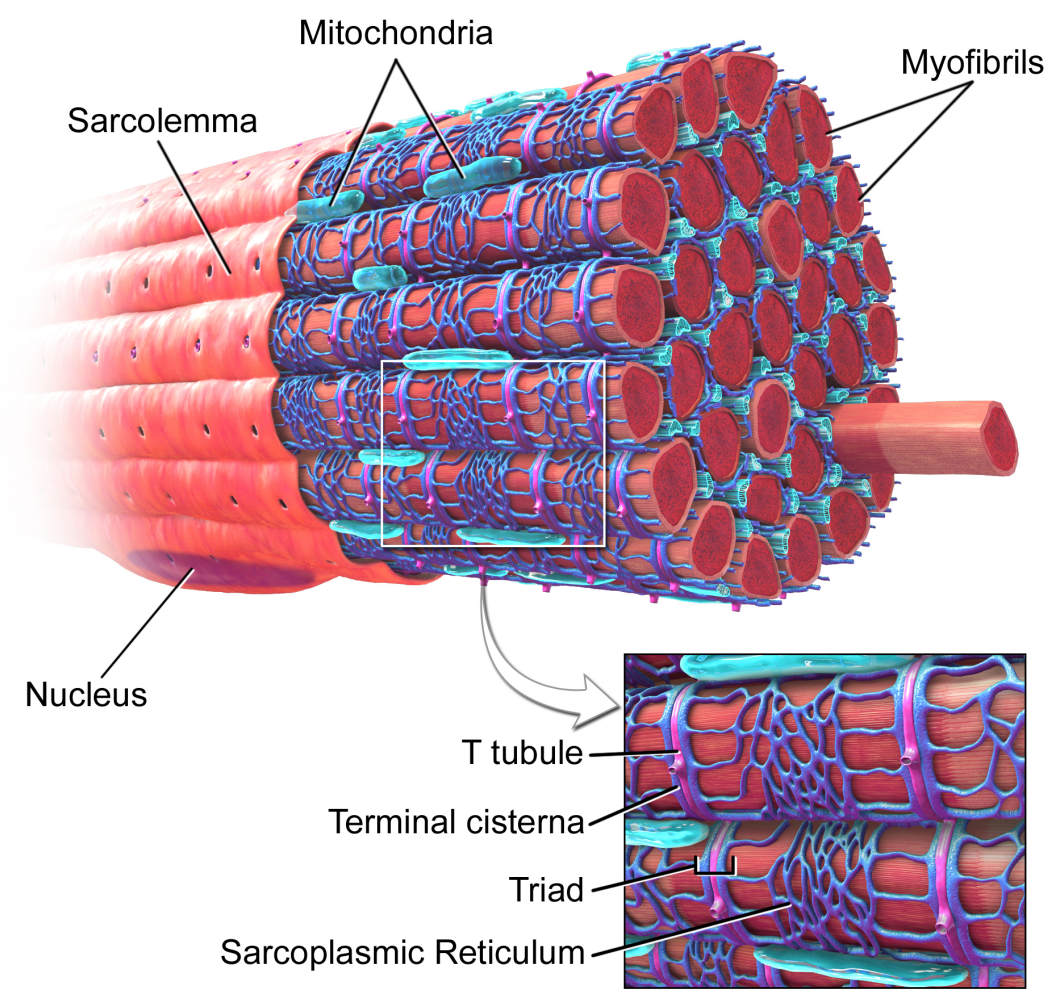

Figure 2.1 - Example of a muscle cell as it can be found in skeletal muscle fibres. Highlighted in purple and pink sarcoplasmic reticulum and t-tubuli are made visible, respectively. These surround the myofibrils and are enclosed by the cell membrane, the sarcolemma. The small figure is an enlargement of a region showing some characteristic SR-T-tubule connecting elements, which represent calcium release units. Figure adopted from [60], Creative Commons, CC BY 4.0.

calcium cycling in myocytes. They are often addressed as Z-discs or Z-lines. These are visible in figure 1.1 where a confocal image of a single cardiomyocyte is shown with a closeup of the Z-lines.

Note that figure 2.1 is a sketch of a skeletal myocyte and therefore differs in some details from cardiac ventricular myocytes, for instance in the location of the nucleus and the form of the cell. Since figure 2.1 serves the purpose of explaining the main components of calcium handling in myocytes only and nucleus and mitochondria are not considered in this study, these differences can be ignored here.

\subsubsection{Calcium Diffusion in Cardiomyocytes}

Calcium is responsible for the proper contraction of cardiomyocytes, in an interplay with troponin $\mathrm{C}$. In order to have a synchronous activation of the myofibrils and therefore allow for optimal functioning of the muscle cell, nature perfected a mechanism that robustly allows high calcium concentrations to be depleted in myocytes in a short 
time window. The interplay of this mechanism with the depolarisation of the cell membrane is known as excitation contraction coupling (ECC). Excitation contraction coupling plays an even more crucial role in cardiac myocytes, since it is the key for a robust functioning and beating of the heart.

\section{Excitation contraction coupling and calcium induced calcium release}

In ventricular cardiomyocytes a depolarisation of the cell membrane, initiated by sodium ions entering the cell, triggers L-type calcium channels (LCCs) that are located on the sarcolemma and specifically on the t-tubuli allowing an inflow of calcium ions $\left(\left[\mathrm{Ca}^{2+}\right]\right)$ from the exterior of the cell. These calcium ions eventually bind to ryanodine receptors 2 (RyR2 or RyR) starting a process called calcium-induced calcium release (CICR). The RyRs, which are located across the LCCs on the jSR (see figure 2.2b), are calcium sensitive and thus more prone to open when the named ions bind to them. Open RyRs release high amounts of calcium ions from the SR, where the concentrations are up to ten orders of magnitude higher than in the cytosol [65-67]. The triggered avalanche of calcium ions, the CICR, is responsible for a fast (from some ten ms in some animal ventricular myocytes $[27,37]$ up to hundreds of $\mathrm{ms}$ in human cardiomyocytes [36]) depletion of a high concentration of ions (usually up to $1.5 \mu \mathrm{M}$ ) into the cytosolic space (that at rest has a $\left[\mathrm{Ca}^{2+}\right]$ of $0.1 \mu \mathrm{M}$ ). Locally, i.e. in the proximity of CRUs, this difference in calcium concentration is even more pronounced; there the depletion from the SR can reach peaks up to hundreds of $\mu \mathrm{M}$ in some tens of ms [27]. This strong and short change in calcium ion concentration inside the myocytes is often addressed to as calcium transient. For a detailed description of CRU geometry and functioning, refer to section 2.1.2.

Once the calcium depletion from the SR is concluded, the ions bind to troponin $\mathrm{C}$ and finally to the actin cross bridge, where myosin heads are then allowed to attach and initiate contraction of the muscle fibres $[14,61-63]$. Afterwards $\left[\mathrm{Ca}^{2+}\right]$ is reabsorbed into the $\mathrm{nSR}$ via the sarco/endoplasmic reticulum $\mathrm{Ca}^{2+}$-ATPase (short SERCA) [67].

\section{The role of SERCA, SR load and NCX}

The process of calcium cycling is completed once the so-called SERCA pumps refill the sarcoplasmic reticulum with free calcium ions $\left[\mathrm{Ca}^{2+}\right]$ from the cytosol. Once the $\mathrm{SR}$ is refilled the process of calcium depletion and contraction is ready to undergo a new cycle.

The amount of calcium stored in the SR determines indirectly how many ions are extruded through the RyRs into the cytosol. The role of SERCA is crucial for the regulation of the calcium content in the SR and is thus often modified in experiments to mimic diseased or malfunctioning cells $[5,19,68]$. The SR calcium load is shown to be crucial for a reliable functioning of ECC and calcium cycling [2]; the strength of SERCA is therefore one of the best measures that can be addressed both computationally and experimentally to alter the ion content in the SR. 
Finally a further component inside myocytes has to be mentioned here, the sodium calcium exchanger (NCX). This membrane potential dependent channel present on the sarcolemma regulates the sodium and calcium content in the cell. Depending on the actual value of the membrane voltage and of the calcium content inside the cytosol this channel either pumps calcium ions into the cell and sodium ions out or vice versa. This channel is known to be a potential source of dysfunction in cardiomyocytes $[16,69]$ and is thus often treated with special attention. Nevertheless in the course of my study the NCX plays a minor role. For this reason I merely mention it here and describe its main modelling parameters and variables below in section 2.2.

\subsubsection{Calcium Release Unit}

The main subcellular component regulating the calcium dynamics inside myocytes can be considered to be the so called calcium release unit (CRU) [70, 71]. This subcellular component is the colocalisation of several types of calcium channels placed at different compartments of the cell itself.

A sketch of the embedding of CRUs in the cellular structure is shown in figure 2.2a, where six CRUs or dyadic clefts are schematically arranged. In the figure also four main channels responsible for the ionic exchange across cellular and subcellular membranes are depicted as green, blue and yellow dots and as red diamonds.

The Ryanodine Receptors 2 (abbreviated with RyR2 or RyR), present in cardiomyocytes, are proteins connecting a net like structure called sarcoplasmic reticulum (SR), which stores calcium ions, to the cytosol. The connection between the cytosol and the external environment of the cell is ensured by the so called L-type Calcium Channels (short LCC - some times dihydropyridin channel, DHC), which are voltage-dependent calcium channels.

The LCCs are directly situated on the cell membrane (sarcolemma), which confines the cell itself. In many myocytes (in particular ventricular cardiomyocytes) the sarcolemma reenters towards the inner parts of the cell via invaginations called transverse tubules (t-tubules), which allow the allocation of LCCs also in the inner parts of the myocyte. Figure 2.2b shows a closeup of one dyadic cleft with LCCs depicted as blue dots and RyRs as red diamonds.

The cytosolic space separating the two types of calcium channels (RyRs and LCCs) is called dyadic space or cleft and typically has a height $15 \mathrm{~nm}[26,72,73]$.

In the very detail the dyadic cleft is part of the broader defined $C R U$; nevertheless I will often use both term as interchangeable, because the distinction between the both only seldomly is of relevance for my study.

\subsubsection{Channel Placements}

The placement of channels, in particular of RyR channels in CRUs, did not play a major role in mathematical models of intracellular calcium dynamics of cardiomyocytes. Specifically the RyRs were assumed to be arranged on a regular lattice and densely packed $[12,27,33,35,44,45,74]$. This assumption has its origin in the lack of 


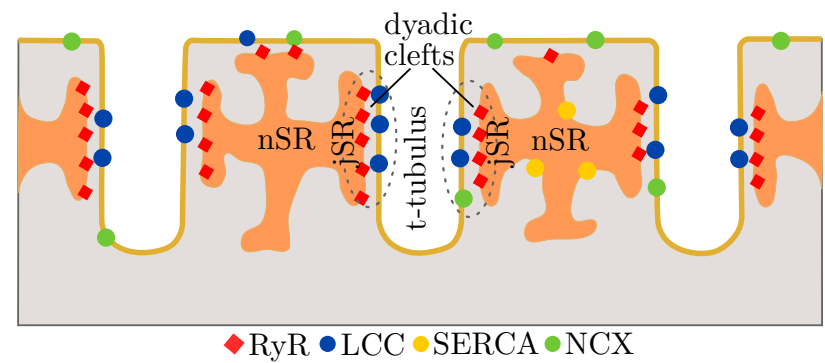

(a) - Sketch of three t-tubuli connecting the cell exterior to the junctional and network sarcoplasmic reticulum (orange shapes). Dyadic clefts are enclosed by dashed ellipses and contain both LCCs and RyRs (blue dots and red diamonds, respectively). On the sarcolemma NCX is highlighted by green dots, while the yellow dots depict SERCA pumps on the nSR.

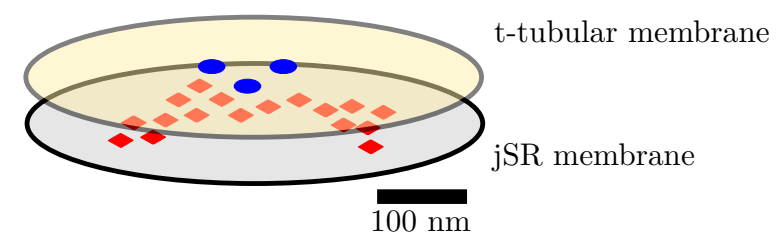

(b) - CRU structure schematically represented by a cylindrical structure. The two circles represent the t-tubular and jSR membrane and are are covered by LCCs (blue dots) and RyRs (red diamonds), respectively.

Figure 2.2 - Sketch depicting the principal components responsible for ECC and CICR in cardiac myocytes. Figure 2.2a shows schematically how CRUs are embedded in the cellular structure, while figure $2.2 \mathrm{~b}$ depicts a close-up up of one dyadic cleft. Adapted from [50], Creative Commons, CC BY-NC-ND 4.0.

information regarding the channel positions from an experimental point of view. Because of their reduced size (some tens of $\mathrm{nm}$ ), measurements to pin down the exact position of single RyRs require a technique that has been developed and achieved only recently [1].

Jayasinghe, Clowsley and coauthors were able to visualise single RyRs using a newly developed microscopy technique called DNA-Paint [22] and were able to describe both the shape and the packing of RyR clusters [1]. In figure 1.2 two sketches of the same cluster are shown. The left figure shows how an RyR cluster is assumed to be formed when classical microscopy techniques are applied to observe it (e.g. dSTORM [75]). These usually posses a resolution that allows for the contours of the cluster to be well defined, but negate a more detailed description of the single channel position. The right sketch in figure 1.2 depicts the same cluster when observed with DNA-Paint. With this technique the group around Christian Soeller was able to observe the position of single molecules inside the RyR cluster and therefore to resolve different and more outlines in it. Of notice is also the different packing and thus density of RyRs inside the cluster.

The group further developed an algorithm for mimicking, in mathematical simulations, the true placement of channels in RyR clusters. This algorithm (detailed here in section 2.2.1) generates RyR clusters that reproduce well the shape and the channel density of those observed in experiments. The authors further ask for mathematical 
models "to be refined to capture the effect of such gaps on excitability using the data provided here" [1].

Since our mathematical model is able to describe both the intra-cellular as well as the intra-dyadic calcium dynamics with detailed channel arrangement in it, we decided to follow the exhortation of the people around Christian Soeller. We therefore included the developed placement algorithm in our cleft model and studied its implications on calcium cycling and excitation contraction coupling.

\subsection{Cleftdyn: Multiscale Model of Intracellular Cal- cium Cycling}

The multiscale model employed in the present thesis consists of three main modules. The model in this work will be often referred to as cleftdyn for the sake of simplicity. The core of development of cleftdyn and its code is done by Martin Falcke from the Max Delbrück Center for Molecular Medicine in Berlin and his collaborators [23, 24, $35,45-47]$.

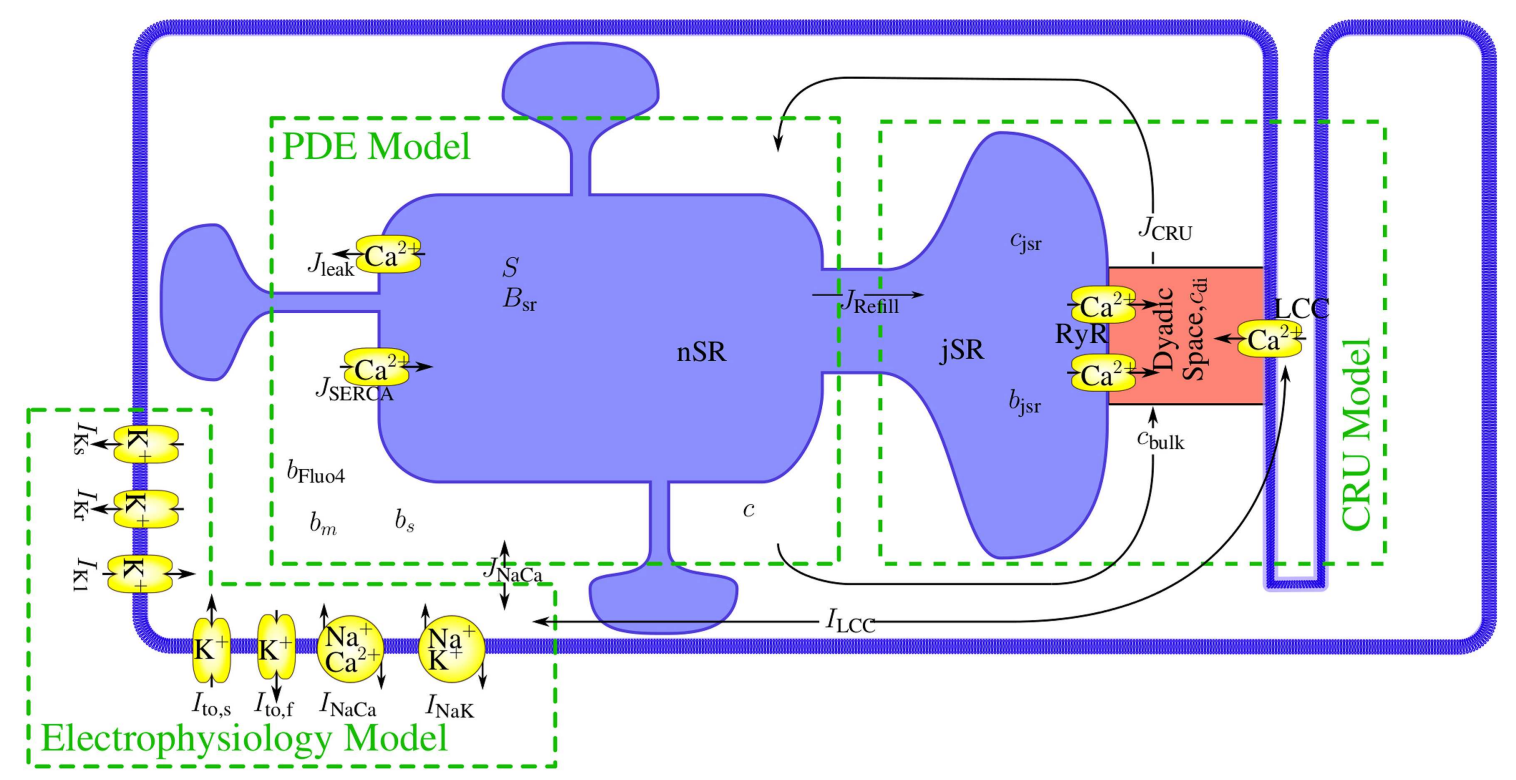

Figure 2.3 - Schematic representation of the main three modules that make up cleftdyn (i.e. the PDE model, the CRU model and the electrophysiology model) separated by green dashed boxes. The blue outer box is a sketch of the cell membrane with a t-tubule invagination. The purple coloured shape represents the sarcoplasmic reticulum and the yellow ion-labelled forms are respective ion channels. The depicted variables, fluxes and currents are described in detail in sections 2.2.2, 2.2.3 and 2.2.4. The figure was adapted from [35, 47], Creative Commons, CC BY 4.0.

The mathematical model is composed of three main modules that in turn are models themselves: the electrophysiology model (i.e. the dynamics of the membrane 
voltage), the partial differential equations (PDE) for calcium diffusion inside the cell and the CRU model. A sketch of how the three modules are incorporated into the model of a cardiomyocyte is shown in figure 2.3. There the three models are framed by green dashed boxes, the main inter-module fluxes are shown together with the variables representing the model's concentrations. For a detailed description of the individual quantities please see sections 2.2.2, 2.2.3 and 2.2.4.

In the following sections I will describe the main components (the three modules), simulation ideas and geometry behind the model.

\subsubsection{Model Geometry}

The multiscale model approximates the cell as a three dimensional square box. Simulations are usually carried out on surroundings of longitudinal cuts of the cell, specifically around the layers, in which CRUs are located. For the sake of simplicity and according to the established naming in literature, these surroundings are called Z-discs (sometimes also Z-lines or Z-disks) [31, 35, 70, 76].

Further the CRUs are spatially resolved and described by a two dimensional disc with a variable radius[24, 35, 47].

\section{Cell interior: cytosol and sarcoplasmic reticulum}

The cytosol, i.e. the cell interior, is assumed to be colocalised with the sarcoplasmic reticulum (SR) and is discretised on the same geometry. A region in space of $15 \times$ $15 \times 2 \mu \mathrm{m}$ I will refer to as full Z-disc, while when referring to four quarter Z-discs the region I consider is of $7.5 \times 7.5 \times 10 \mu \mathrm{m}$, i.e. four quarters of a full Z-disc "stacked" on top of each other.

Every Z-disc has one plane with equally distributed CRUs at the central z coordinate, meaning that for instance a $2 \mu \mathrm{m}$ thick Z-disc has the mentioned plane at $z=1 \mu \mathrm{m}$. The virtual plane on which the CRUs are placed is considered to be the region of the invaginations by the t-tubules.

At the boundaries of the cell interior von Neumann boundary conditions are applied (see equations (2.5) and (2.6)). To avoid resonant behaviour at the boundaries because of the chosen conditions, the CRUs at the corner positions on the plane are not considered. Unless otherwise stated, the full Z-disc has $320 \mathrm{CRUs}$, while on a quarter Z-disc only 78 of them reside, resulting in 312 CRUs for the case of four quarter Z-discs.

An example of a full Z-disc is shown in figure 2.4, where a top view on the $\mathrm{x}-\mathrm{y}$ plane is depicted together with 320 CRUs as blue dots. Notice the missing CRUs at the Z-disc corners.

\section{Calcium Release Unit: cleft and channel properties}

The calcium release unit (CRU) is the overall aggregate of t-tubule, LCCs, RyRs and jSR. The former two are facing the latter ones and are separated by a gap called dyadic cleft or space (see section 2.1.2 for details). 


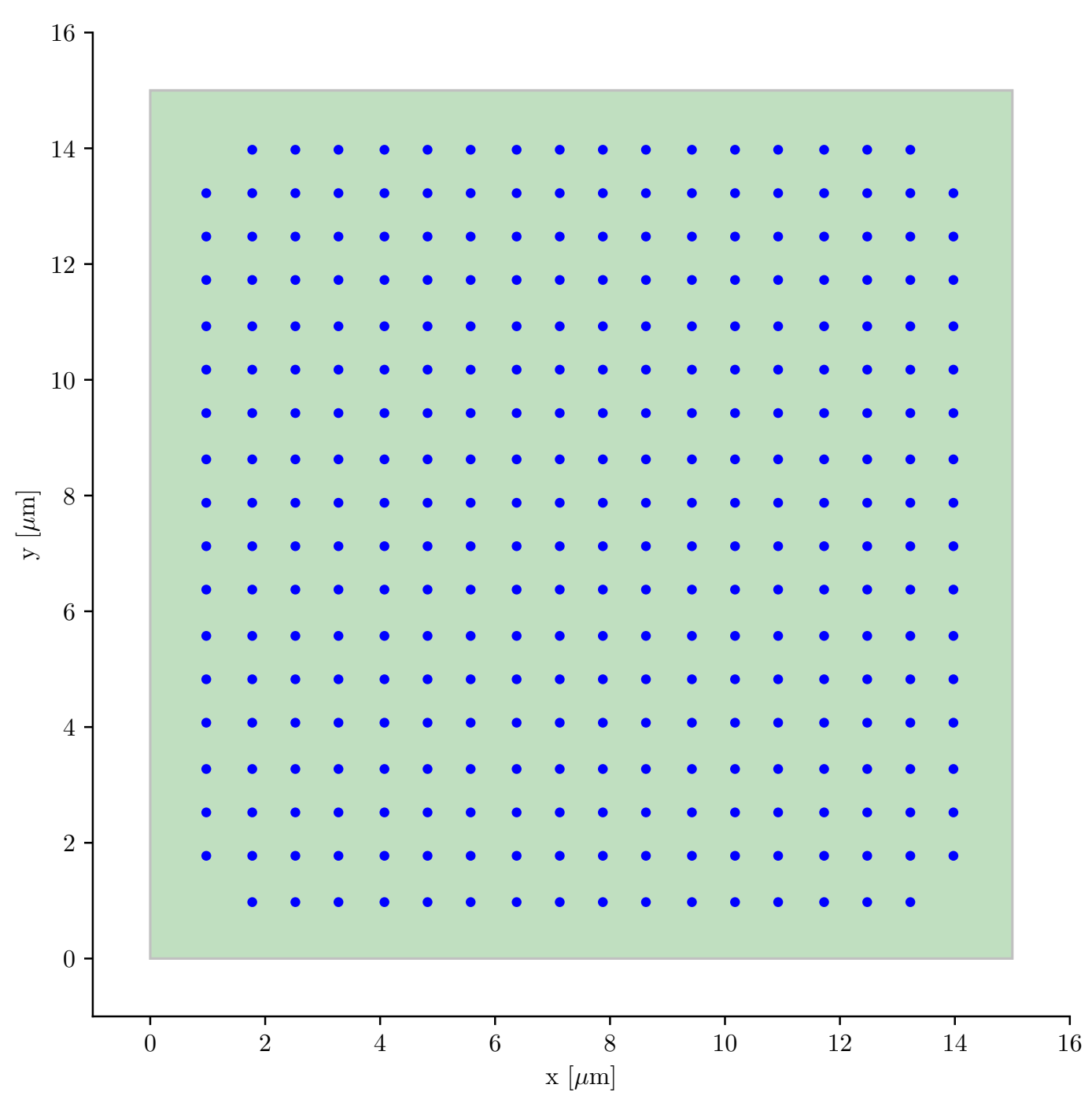

Figure 2.4 - Top view of a standard Z-disc with CRUs depicted as blue dots.

The dyadic cleft is described as a cylinder of fixed height and variable radius. The latter strongly depends on the total number of channels contained in the CRU. The radius of the cleft underlies two assumptions; first it is chosen to be smaller than $300 \mathrm{~nm}$ and/or it has a minimal distance of $60 \mathrm{~nm}$ from the outermost channel [35, 47].

The number of RyRs is drawn randomly from an exponential distribution with average 20 RyRs at the initialisation of each simulation and for each cleft separately. Further a lower and upper bound for the total number of RyRs is fixed at 9 and 80 RyRs, respectively. The resulting number of RyRs then determines the total number of LCCs of a given cleft via a predefined ratio of the channel numbers (RyR/LCC) fixed at the value of 5 , unless stated otherwise.

As described in section 2.1.3, the state of the art sees two types of arrangement of the RyRs; the regular, lattice like placement $[12,24,27,35]$ and the random walk placement [1]. 


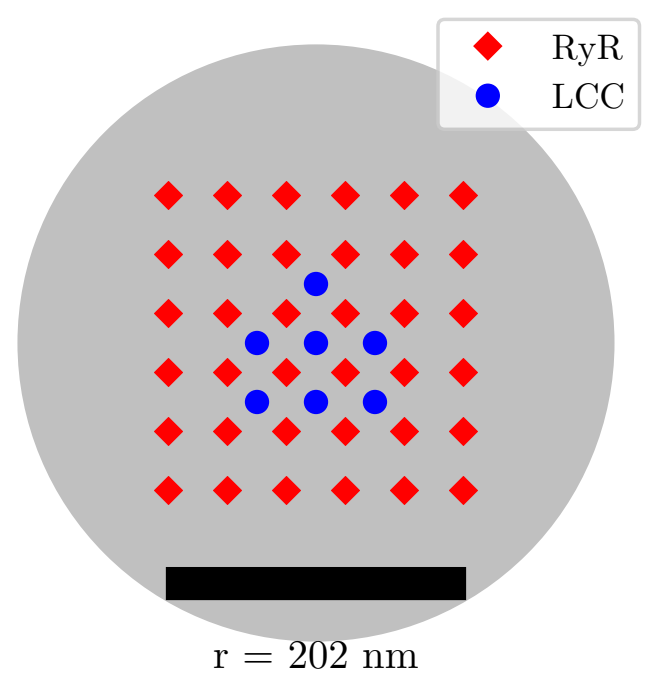

(a) - Top view of a single cleft with regular placed RyRs.

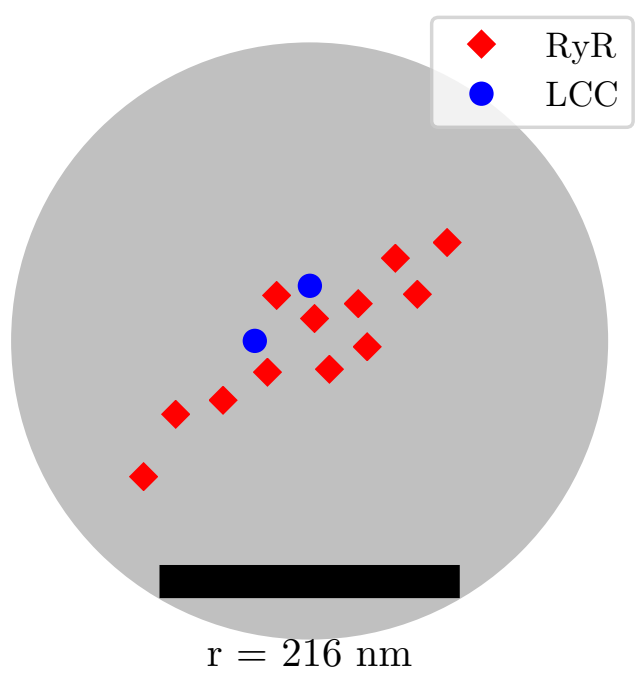

(b) - Bottom view of a single cleft with Soeller placed RyRs.

Figure 2.5 - Two examples of placement from different algorithms for ryanodine receptors (red diamonds). L-type calcium channels are marked as blue dots. The black scale bar with its label describes the cleft radius.

The regular placement is described in detail in [45]. A regular grid of equidistant $(40 \mathrm{~nm})$ channels is generated using the number of RyR drawn from the exponential distribution and the number of LCC computed from the RyR/LCC ratio as described above. In this process LCCs are chosen not to be colocalised with RyRs and both channel clusters have their centre of mass at the origin of the circles that surround the cleft. An example of such placement is depicted in figure 2.5a, where RyRs are depicted as red diamonds and LCCs as blue dots. The cleft is shown as a grey circle with radius highlighted by the black scale bar.

The random walk placement I will also refer to as the Soeller or Jayasinghe placement in the following. It is implemented by a random walk that starts from the centre of the cleft and places the following RyR at a distance that is randomly drawn from a Gaussian distribution and with a random angle in the interval $[0,2 \pi)$. The placement algorithm is explained in [1] and [50]. The Soeller placement algorithm considers excluded volumes, i.e. it pays attention not to place RyRs on top of each other by placing channels at a minimal distance of $30 \mathrm{~nm}$ from already placed ones.

An example of the Soeller-like placed channels can be seen in figure 2.5b, where RyRs are highlighted with red diamonds and LCCs with blue dots. The cleft is depicted as a grey disc with radius specified below the black scale bar. As it is shown here, the Soeller placement algorithm generates RyR clusters irregular in shape compared with the ones that arise from the regular placement. 
Mathematical Model and Methods

\subsubsection{Electrophysiology}

The electrophysiology of the cell is described by a well known and widely spread ionic model known as Mahajan model [37]. This model based on ordinary differential equations describes the time evolution of intracellular ions (e.g. calcium and sodium) together with the related ion membrane currents and the resulting action potential of one single rabbit ventricular myocyte.

The original Mahajan model [37] considers the inherent stochasticity of LCCs with a Markovian model that describes the different open, inactive or closed states of the given channel. The inclusion of the Mahajan model in the present state of the multiscale model was adapted in order to allow for a separate, though in its form similar, treatment of the channel openings [35, 47]. The mechanism of channel opening is described in detail in section 2.2.4.

The Mahajan model employed in the present version of the multiscale model was further adapted in some ion concentrations and currents in order to allow the connection to the spatially resolved modules of the model. These changes concern the current through the sodium-calcium exchanger NCX $I_{\mathrm{NaCa}}$, the L-type calcium channel current $I_{\mathrm{CaL}}$ and the action potential $V$. The first one is linked to the NCX flux $J_{\mathrm{NaCa}}$ in the PDE module (see section 2.2.3, the second contributes via the CRU module (section 2.2.4) and the CRU flux $J_{\mathrm{CRU}}$ (equation (2.7)) to the calcium PDE (2.3) of the cytosol model. The action potential $V$ finally is used to compute the LCC current itself (see equation (2.18)).

The Mahajan model assumes a spatially uniform membrane potential $V$ that evolves in time according to:

$$
C_{m} \frac{\mathrm{d} V}{\mathrm{~d} t}=-\left(I_{\text {ion }}+I_{\text {stim }}\right)
$$

where $I_{\text {stim }}$ is the current depolarising the cell membrane, $C_{m}$ is the membrane capacitance and $I_{\text {ion }}$ is the sum of several ion currents:

$$
I_{\mathrm{ion}}=I_{\mathrm{Na}}+I_{\mathrm{to}, \mathrm{f}}+I_{\mathrm{to}, \mathrm{s}}+I_{\mathrm{Kr}}+I_{\mathrm{Ks}}+I_{\mathrm{K} 1}+I_{\mathrm{NaK}}+I_{\mathrm{CaL}}+I_{\mathrm{NCX}}
$$

where " $I_{\mathrm{Na}}$ is the fast $\mathrm{Na}^{+}$current, $I_{\mathrm{to}, \mathrm{f}}$ is the fast and $I_{\mathrm{to}, \mathrm{s}}$ the slow component of the rapid outward $\mathrm{K}^{+}$current, $I_{\mathrm{Kr}}$ is the rapid delayed rectifier current, $I_{K s}$ is the slow delayed rectifier current, $I_{\mathrm{K} 1}$ is the inward rectifier current, and $I_{\mathrm{NaK}}$ is the $\mathrm{Na}^{+} / \mathrm{K}^{+}$ pump current" [47] and Supporting Material of [50]. These currents are detailed in [37] and are computed in the electrophysiology module itself; two further currents are computed separately, i.e. the sodium-calcium-exchanger (NCX) current $I_{\mathrm{NCX}}$ and the LCC current $I_{\mathrm{CaL}}$. The former is given by the spatial integral of the corresponding, local NCX fluxes over cytosol and cell boundaries (see section 2.2.3 and equations (2.3) and (2.5)), while the latter is determined by the sum of all the single LC channel currents from the CRU module (section 2.2.4). 


\subsubsection{Ion Diffusion}

The following description of the module outlining the PDE model of calcium diffusion in the cytosol is adapted from previous ones $[23,35,47]$ and in the Supporting Material of [50].

\section{Cytosolic domain}

The core module around which cleftdyn is centred is a reaction diffusion model that describes the ion diffusion in the cytosol and sarcoplasmic reticulum. Specifically the dynamics of the concentration of calcium ions $\left[\mathrm{Ca}^{2+}\right]$, which in the following will be denoted by $c$, is described. The calcium diffusion is influenced by the uptake and release by sarcolemma, SR and buffer molecules. The first one happens through LCCs and NCX, the second via RyRs and SERCA pump and the last one via binding to buffer molecules (with its own PDEs).

The calcium-binding buffers $\left(b_{j}, j \in\{s, m, f\}\right)$ considered are the following: troponin $\mathrm{C}$ as stationary buffer $(s)$, calmodulin as mobile buffer $(m)$ and Fluo-4 $(f)$ used in experiments to highlight $\left[\mathrm{Ca}^{2+}\right]$. Total concentrations $\left(b_{j}^{\text {tot }}\right)$ are considered to be spatially homogeneous in the entire cytosolic domain [47].

The modelling PDEs for calcium and buffering molecule concentrations are thus given by:

$$
\begin{aligned}
\frac{\partial c}{\partial t} & =\nabla \cdot(\boldsymbol{D} \nabla c)+J_{\mathrm{CRU}}+J_{\mathrm{NCX}}+J_{\text {leak }}-J_{\mathrm{pump}}-\sum_{j \in\{s, m, f\}} R_{j}\left(c, b_{j}\right) \\
\frac{\partial b_{j}}{\partial t} & =\nabla \cdot\left(\boldsymbol{D}_{b}^{j} \nabla b_{j}\right)+R_{j}\left(c, b_{j}\right)
\end{aligned}
$$

with von Neumann boundary conditions:

$$
\begin{aligned}
\left.\hat{n} \cdot \boldsymbol{D} \nabla c\right|_{\Gamma} & =J_{\mathrm{NCX}}^{p m} \\
\left.\hat{n} \cdot \boldsymbol{D}_{b}^{j} \nabla b_{j}\right|_{\Gamma} & =0 .
\end{aligned}
$$

The buffers $j$ are chosen among $\{s, m, f\}, \boldsymbol{D}$ and $\boldsymbol{D}_{b}^{j}$ are diagonal diffusion matrices and the boundary $\Gamma$ represents the sarcolemma, which in the case of quarter Z-discs coincide with the domain boundaries. The von Neumann boundary condition infers the NCX flux through $J_{N C X}^{p m}$, while the remaining fluxes are defined by: 


$$
\begin{aligned}
J_{\mathrm{CRU}} & =\sum_{i=1}^{N_{c}} \frac{\nu_{\mathrm{cell}}}{\nu_{\mathrm{cyt}}} \frac{\Theta\left(R_{\mathrm{CRU}}^{i}-\left|\boldsymbol{r}-\boldsymbol{r}_{i}\right|\right)}{\frac{4}{3} \pi\left(R_{\mathrm{CRU}}^{i}\right)^{3}}\left[\sum_{k=1}^{N_{\mathrm{LCC}}^{i}} I_{\mathrm{LCC}}^{i, k}(t)+\sum_{k=1}^{N_{\mathrm{RyR}}^{i}} I_{\mathrm{RyR}}^{i, k}(t)\right] \\
J_{\text {leak }} & =V_{l}(S-c) \\
J_{\text {pump }} & =\frac{V_{\mathrm{p}, \max } c^{2}}{K_{p}^{2}+c^{2}} \\
R_{j}\left(c, b_{j}\right) & =k_{j}^{+}\left(b_{j}^{\mathrm{tot}}-b_{j}\right) c-k_{j}^{-} b_{j}
\end{aligned}
$$

Here $\Theta(x)$ is the Heaviside step function ensuring the CRU flux $J_{\text {CRU }}$ is present only at the position of the $i$-th CRU $\boldsymbol{r}_{i}$ with respective radius $R_{\mathrm{CRU}}^{i}$. Each of the $N_{c}$ CRUs has a total number of LCCs and RyRs denoted by $N_{\mathrm{LCC}}^{i}$ and $N_{\mathrm{RyR}}^{i}$ respectively as well as currents defined for every $k$-th channel $I_{\mathrm{LCC}}^{i, k}(t)$ and $I_{\mathrm{RyR}}^{i, k}(t)$ respectively. These are computed according to the stochastic CRU model described in section 2.2.4. Finally in $J_{\mathrm{CRU}}$ a scaling volume ratio $\nu_{\text {cell }} / \nu_{\text {cyt }}$ ensures, together with the $\nu_{\text {cyt }} / \nu_{\text {sr }}$ ratio described below, the cell volume is filled continuously.

This flux represents the main link to the CRU module, in which the intradyadic calcium dynamics together with the channel currents are computed.

The leak flux $J_{\text {leak }}$ describes a flux from the SR into the cytosol and depends through a scaling factor $V_{l}$ on the difference between cytosolic calcium concentration $c$ and SR calcium concentration $S$ (see below).

SERCA pump flux describes the strength with which the SR is reabsorbing calcium ions from the cytosol and is given by a Hill equation that scales with a maximum uptake strength $V_{\mathrm{p}, \max }$, has a dissociation constant $K_{p}^{2}$ and is dependent on cytosolic calcium concentration $c$.

Finally $k_{j}^{+}$and $k_{j}^{-}$are the binding and unbinding rates respectively for the $j$-type buffer.

\section{Sarcoplasmic reticulum}

The ion diffusion is not confined to the cytosolic space only but comprises the sarcoplasmic reticulum (SR) as well. There two further PDEs describe the diffusion of calcium ions and calsequestrin in the SR denoted with $S$ and $B_{\text {sr }}$ respectively.

$$
\begin{aligned}
\frac{\partial S}{\partial t} & =\nabla \cdot\left(\boldsymbol{D}_{S} \nabla S\right)-k_{\mathrm{sr}}^{+}\left(B_{\mathrm{sr}}^{\mathrm{tot}}-B_{\mathrm{sr}}\right) S+k_{\mathrm{sr}}^{-} B_{\mathrm{sr}}-J_{\mathrm{jsr}}+\frac{\nu_{\mathrm{cyt}}}{\nu_{\mathrm{sr}}}\left(J_{\mathrm{pump}}-J_{\text {leak }, \mathrm{sr}}\right) \\
\frac{\partial B_{\mathrm{sr}}}{\partial t} & =\nabla \cdot\left(\boldsymbol{D}_{B} \nabla B_{\mathrm{sr}}\right)+k_{\mathrm{sr}}^{+}\left(B_{\mathrm{sr}}^{\mathrm{tot}}-B_{\mathrm{sr}}\right) S-k_{\mathrm{sr}}^{-} B_{\mathrm{sr}}
\end{aligned}
$$

Both SR concentrations are subject to von Neumann boundary conditions at $\Gamma$, mathematically meaning $\left.\hat{n} \cdot \boldsymbol{D}_{S} \nabla S\right|_{\Gamma}=0=\left.\hat{n} \cdot \boldsymbol{D}_{B} \nabla B_{\mathrm{sr}}\right|_{\Gamma}$. The diffusion matrices are 
given by $\boldsymbol{D}_{S}$ and $\boldsymbol{D}_{B}$, while the binding and unbinding rates of calsequestrin $B_{\text {sr }}$ are described by $k_{\mathrm{sr}}^{+}$and $k_{\mathrm{sr}}^{-}$respectively.

The pump flux $J_{\text {pump }}$ is the direct link to the cytosolic calcium diffusion with equations (2.3) and (2.9), while $J_{\text {jsr }}$ and $J_{\text {leak,sr }}$ are SR specific fluxes that are linked to intra jSR calcium and CRU dynamics (via the channel current $I_{\mathrm{RyR}}^{k}$ elaborated in section 2.2.4). These are not further elucidated here, since they are out of scope for the present study. The details about the calcium dynamics in the jSR can be found in $[35,47]$ and in the Supporting Material of [50].

As mentioned above the cell volume is divided into two main domains, thus in order to fill it continuously volume ratios for different fluxes have to be considered. For the direct interaction between SR and cytosol $\left(J_{\text {pump }}\right.$ and $\left.J_{\text {leak,sr }}\right)$ a scaling with $\nu_{\text {cyt }} / \nu_{\text {sr }}$ is necessary.

\section{Fluorescent buffer}

The already introduced fluorescent buffer Fluo-4 was incorporated into cleftdyn to allow a direct comparison to experiments using fluorescent dyes to visualise calcium ions. To account for the information on how much calcium ions are buffered by Fluo- 4 the approach described in [77] is used:

$$
\left[\mathrm{Ca}^{2+}\right]_{i}^{\exp }=K_{d} \frac{F-F_{\min }}{F_{\max }-F}
$$

"where $K_{d}$ is the dissociation constant of Fluo- $4, F$ is the experimentally measured fluorescence intensity, here the spatial average of $b_{f}, F_{\max }$ is the measured fluorescence intensity in calcium-saturated dye, here this is set as $b_{f}^{\text {tot }}$, and $F_{\min }$ is the measured fluorescence intensity in the absence of calcium, here set to zero" (see equation (3) and describing text in [50]).

The usage of the Fluo- 4 buffer and of the so-called experimental calcium stems from the need to quantitatively compare results of the mathematical model with those from experiments. This will affect mostly measures as for instance peak calcium concentration and full duration at half maximum of calcium sparks detailed in section 3.1.2. In this study we thus opted to focus the analysis on Fluo-4 buffered calcium, which we usually labelled with an exp to emphasise the relation to experimentally measured calcium concentration. Whenever free calcium was used for the analysis, it is stated there.

\subsubsection{Cleft Dynamics}

The calcium dynamics inside each CRU are considered separately from the main PDE model. Inside each cleft two main processes can be differentiated; the stochastic gating of the calcium channels and the actual calcium diffusion in the dyadic space. 
Mathematical Model and Methods

\section{Markov chains and stochastic channel gating}

Calcium channels in cleftdyn are allowed to be in different states mimicking the different configurations that the molecule can assume. To simulate this behaviour Markov chains are well suited and often used [12, 23, 27, 37, 78-80].

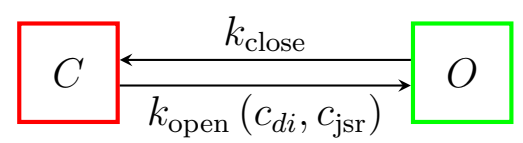

Figure 2.6 - Markov chain representing the closed and open states of RyRs [12]. The open rate depends on dyastolic as well as jSR calcium as described in equation (2.13). Adapted from [50], Creative Commons, CC BY-NC-ND 4.0.

Most of the Markov chain models describing the channel gating have in common that one open state $O$ is present and at least one closed $C$ and/or inactivated $I$ state. The latter are assumed to allow no calcium flux through the channel itself, while the open state allows it.

Previous implementations and versions of cleftdyn considered different Markov models $[24,35,47]$. In this work I employed only two different models, i.e. the Walker model for the RyR gating and the Mahajan model for the LCC gating. These are depicted in figures 2.6 and 2.7 respectively. The open state $O$ is highlighted by a green square, while the other states are boxed in red.

The transition rate constants of the Mahajan Markov chain models can be found in table A.4 and in [37]. Particularly interesting are the transition rates defined in the Walker model; while the closing rate is constant $k_{\text {close }}$ the opening one depends on both intradyadic and jSR calcium concentration $\left(c_{d i}\right.$ and $c_{\mathrm{jsr}}$ respectively):

$$
k_{\text {open }}=k_{\text {plus }} c_{d i}^{\eta}\left[\phi_{b}+\left(\frac{c_{\mathrm{jsr}}}{\phi_{k}}\right)^{4}\right] .
$$

Where $k_{\text {plus }}$ is truly a rate constant, $\phi_{b}$ and $\phi_{k}$ are regulatory constants and $\eta$ is the Hill coefficient that describes the calcium sensitivity of the RyR channels. The last three constants can be found in table A.2.

Equation (2.13) introduces the calcium sensitivity of the RyRs into the model and therefore allows for the calcium induced calcium release.

The determination of the time steps between transitions and the respective channel states is generally ruled in mathematical models by an efficient algorithm named after its inventor, David Gillespie [81-83]. The Gillespie algorithm was originally applied to chemical reactions, but is extendable to reactions with time dependent rates as well and thus is applicable to Markov chains.

The Gillespie algorithm chooses the next transition in a reaction chain by computing probabilities from each rate and finding the smallest transition time. It thus efficiently 


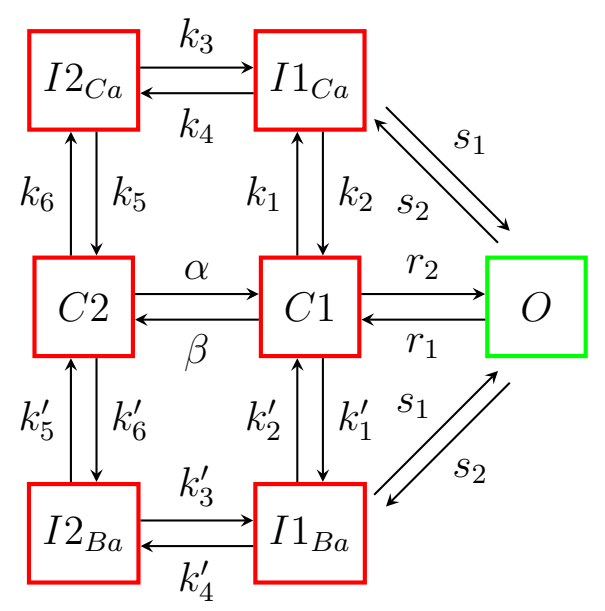

Figure 2.7 - Markov chain depicting the Mahajan model for closed, inactive and open states of the L-type calcium channels [37]. Adapted from [50], Creative Commons, CC $B Y-N C-N D$ 4.0.

determines the time scale for the stochastic gating. As a result of the minimisation of the transition times, very small time steps can be inferred. In cleftdyn, where bigger time scales (of the PDE and electrophysiology model) are present as well, the Gillespie algorithm is implemented in a way that advantage is gained from the inactive and closed states of the Markov chains. These do not contribute to a calcium flux through the channels, therefore only transitions to and from the open state are considered to be dependent stochastic events and evaluated in the frame of the PDE model.

\section{Intradyadic calcium diffusion}

In the following I will sketch the derivation of the calcium concentration profile inside the dyadic cleft. I mainly adopted this from previously described derivations of the same [24, 35, 43, 47]; this description can be found in [50] and in the associated Supporting Material as well.

The profile of the calcium concentration in the $i$-th cleft $c_{d i}$ is described by a partial differential equation in cylindrical coordinates [24]:

$$
\frac{\partial c_{d i}}{\partial t}=\sum_{k=1}^{N_{\mathrm{LCC}}^{i}} I_{\mathrm{LCC}}^{k} \delta\left(\boldsymbol{r}-\boldsymbol{r}_{\boldsymbol{k}}\right)+\sum_{k=1}^{N_{\mathrm{RyR}}^{i}} I_{\mathrm{RyR}}^{k} \delta\left(\boldsymbol{r}-\boldsymbol{r}_{\boldsymbol{k}}\right)+D_{c} \Delta_{r, \varphi} c_{d i}(\boldsymbol{r})-\frac{\partial}{\partial z} J_{z}
$$

where $N_{\mathrm{LCC}}^{i}$ and $N_{\mathrm{RyR}}^{i}$ are the total number of LCCs and RyRs, respectively, and $I_{\mathrm{LCC}}^{k}$ and $I_{\mathrm{RyR}}^{k}$ their ion currents at the $k$-th channel mouth. The Dirac-delta function ensures that an ion current is present only at the channel positions $\boldsymbol{r}_{\boldsymbol{k}} . D_{c}$ is the diffusion constant of calcium in the dyadic space. $J_{z}$ finally is a flux that takes into account the electro-diffusion between membranes as well as their buffering.

A separation ansatz, a steady state assumption, an integration in $z$ direction and 
the application of Green's function lead to a stationary solution for the calcium profile in the cleft (see [35, 43] and Supporting Material of [50]):

$$
c_{d i}(\boldsymbol{r})=Z\left(z_{i}\right) c_{\mathrm{bulk}}+\sum_{k=1}^{N_{\mathrm{LCC}}^{i}} I_{\mathrm{LCC}}^{k} \eta\left(\boldsymbol{r}_{k}, \boldsymbol{r}\right)+\sum_{k=1}^{N_{\mathrm{RyR}}^{i}} I_{\mathrm{RyR}}^{k} \eta\left(\boldsymbol{r}_{k}, \boldsymbol{r}\right)
$$

with the abbreviations:

$$
\eta\left(\boldsymbol{r}_{k}, \boldsymbol{r}\right)=\frac{Z(z)}{\beta_{d} h^{*} D_{c}} G\left((r, \phi),\left(r_{k}, \phi_{k}\right)\right)
$$

and $h^{*}=\int_{0}^{h} d z Z(z)$. Here $Z(z)=\exp \left(-2 \phi_{0} \exp (-\kappa z)\right)$ is the electrodiffusion gradient first established by Soeller and Cannell [72] and used in [24, 35, 43, 47]. The calcium concentration at the boundary of the cleft is described by $c_{\text {bulk }}$. The constants $\phi_{0}, h$, $\kappa$ and $\beta_{d}$ are listed in table A.5. Moreover, the Green function $G\left((r, \phi),\left(r_{k}, \phi_{k}\right)\right)$ is defined by:

$$
\begin{aligned}
& G\left(\boldsymbol{r}, \boldsymbol{r}_{\boldsymbol{k}}\right)=\frac{1}{2 \pi} \ln \left(\frac{\left\|\hat{\boldsymbol{r}_{k}}-\boldsymbol{r}\right\|}{q\left\|\boldsymbol{r}_{\boldsymbol{k}}-\boldsymbol{r}\right\|}\right) \text { for } \boldsymbol{r}_{k} \neq 0 \\
& G(\boldsymbol{r}, 0)=\frac{1}{2 \pi} \ln \left(\frac{R}{\|\boldsymbol{r}\|}\right),
\end{aligned}
$$

where $q=R /\left\|\boldsymbol{r}_{\boldsymbol{k}}\right\|, R$ is the cleft radius and $\hat{\boldsymbol{r}_{\boldsymbol{k}}}=q^{2} \boldsymbol{r}_{\boldsymbol{k}}$.

The channel currents $I_{\mathrm{LCC}}^{k}$ and $I_{\mathrm{RyR}}^{k}$ are dependent on the calcium concentrations present in the dyadic space $c_{d i}$, in the $\mathrm{jSR} c_{\mathrm{jsr}}$ and in the exterior of the cell $c_{\mathrm{ext}}[35$, 47]:

$$
\begin{aligned}
& I_{\mathrm{RyR}}^{k}=J_{R}\left(c_{\mathrm{jsr}}-c_{d i}\left(\boldsymbol{r}_{k}\right)\right) \\
& I_{\mathrm{LCC}}^{k}=J_{L} V \delta \frac{c_{\mathrm{ext}} \mathrm{e}^{V \delta}-c_{d i}\left(\boldsymbol{r}_{k}\right)}{1-\mathrm{e}^{V \delta}} .
\end{aligned}
$$

The concentrations $c_{\text {ext }}$ is the as constant assumed calcium concentration in the cell exterior, while $c_{\mathrm{jsr}}$ is computed as part of the jSR dynamics (section 2.2.3). The membrane potential $V$ links the CRU module to the electrophysiology model (section 2.2.2) and $\delta=2 F /\left(R_{B} T\right)$ comprises several constants: Faraday constant $F$, universal gas constant $R_{B}$ and temperature $T=308 \mathrm{~K}$. Important measures here are $J_{R}$ and $J_{L}$ representing the permeability of the respective channel and a parameter that can be altered to change the allover strength of currents. The former I often address as $g_{\text {RyR }}$ and the latter as $g_{\mathrm{LCC}}$. These permeabilities are two of the crucial parameters that I varied in this study (see section 3.1.3). 
Together with equation (2.15) a system of linear equations can now be established for $c_{d i}$ and $c_{\text {bulk }}$. In combination with the Markov chain model ensuring the state of every single channel, the calcium gradient inside each CRU can ultimately be computed.

\subsubsection{Remarks on Computational Costs}

The multiscale nature of cleftdyn both in its time steps and geometry (schematically shown in figure 2.8) together with the generation of pseudo random numbers makes each simulation run very costly. The code is implemented in $\mathrm{C}++$ and makes usage of a finite element method library called DUNE (acronym for Distributed and Unified Numerics Environment) [84-86]. This library allows the parallelisation of the code on several CPUs employing the well known message passing interface (MPI) [87], which remarkably increases the speed of the computation.

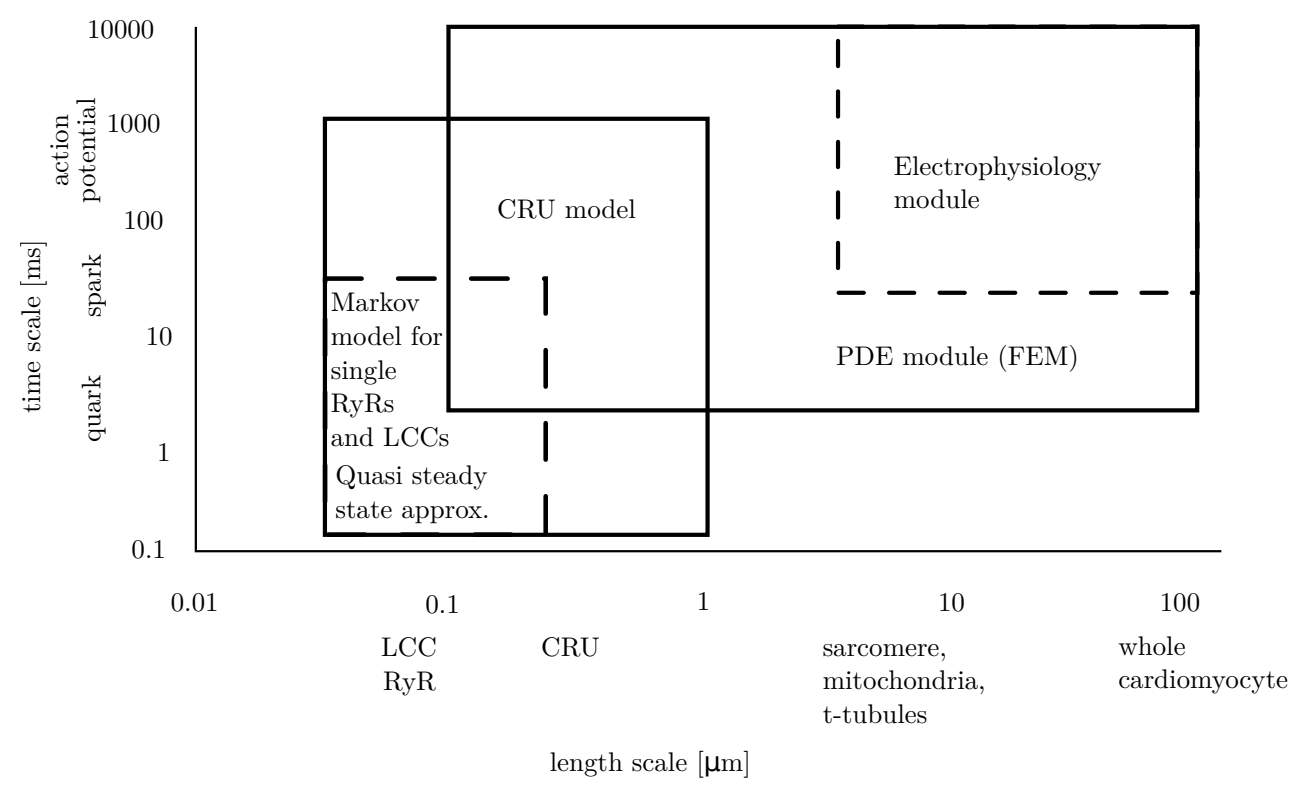

Figure 2.8 - Schematic representation of the spatio-temporal orders of magnitude covered by cleftdyn. The temporal scales, here on the y-axis, range from single channel openings, i.e. quarks (see section 3.1.1 for details), in a range of some milliseconds up to series of action potential along several seconds. The spatial resolution ranges from single calcium channels in the order of some tens of nanometers up to the extension of one entire cardiomyocyte at around hundred micrometers. The boxes include the different modules that construct cleftdyn (see section 2.2 for details). Adapted from Supporting Material of [50], Creative Commons, CC BY-NC-ND 4.0.

Nevertheless a single simulation run of one action potential simulation on one Z-disc (see section 3.1.1 for details) with a simulation time of $5250 \mathrm{~ms}$ parallelised on 400 Skylake (SKL) CPU-cores with $2.4 \mathrm{GHz}$ needs 18 to $24 \mathrm{~h}$ computation time to terminate.

Considering these demanding simulation costs methods for relevant and robust parameter analysis based on a small amount of performed runs are needed. Further 
adopting surrogate models capable of mimicking the basic features in selected input and output regions of the model can help conducting subsequent studies lowering the computational costs.

In the following chapter the first simulation results are shown together with the tools used to analyse them. The focus lies on methods around uncertainty quantification, which allowed us to perform a robust parameter analysis based on the statistically small amount of simulation runs that can be performed with cleftdyn. 


\section{Chapter 3}

\section{Parameter Analysis}

The influence the geometry of dyadic clefts has on the cell's functions can be investigated with model parameters that are linked to the calcium handling in CRUs; in the following I will call these parameters input or selected parameters.

The mathematical model used here, i.e. cleftdyn, is computationally very demanding (see section 2.2.5). Since the input parameter space is high dimensional (in this work it has five dimensions), it is not feasible to perform a fine scan of it with such a computationally expensive model. Phrased differently, the high dimensionality requires a great number of simulation runs, which is impeded by the expensive computational costs. In this study we thus employed statistical methods to allow a determination of ranges of input parameters using few numbers of simulation runs [51, 52].

The chapter focuses on the description of these methods (linear sensitivity analysis, polynomial chaos expansion) and shows their application to cleftdyn. The processes described here represent the foundation, on which the results outlined in section 4.1 and presented in [50] are based.

\subsection{Generating Model Populations}

The general approach with which data were collected from simulations was the generation of model populations, i.e. a set of simulation runs characterised by the variation of the selected input parameters. Since the model has stochastic components both in the construction of the geometry (see section 2.2.1) and in the channel gating (see section 2.2.4) different output can be expected from simulation runs with the same initial parameter set.

A variation in the input parameter set and an analysis of the robustness of the model with respect to changes in the initial conditions allows to address and describe the impact of changes in the subcellular structure and functions on ECC and cardiomyocyte functionality in general. Since we are interested in the effects of channel functionality and placement on the model's output, we chose the parameters to be varied in a range that best describes their physiological functions (see section 3.1.3 for details). 
The procedure of creating model populations was adopted for two different simulation modes, which each yield different outputs also called biomarkers. The following sections 3.1.1 and 3.1.2 describe the simulation types and the biomarkers in detail.

\subsubsection{Simulation Types}

For cleftdyn two main simulation schemes best lead to a broad description of the intracellular dynamics. We called the two procedures action potential (short AP) and spark simulations.

AP simulations are usually performed on full Z-discs, rarely on quarter Z-discs for testing purposes (the results shown in this thesis are all with full Z-discs). In AP simulations the cardiomyocyte is stimulated with periodic action potentials. Each AP initiates an ECC-cycle that leads to the contraction of the cell. This mimics an experiment in which a cardiomyocyte is externally driven by an electrical stimulus. The pacing cycle length (short PCL), i.e. the interval between two stimuli, was chosen to be PCL $=350 \mathrm{~ms}$. We conducted several tests for different PCLs and compared these with those found in literature $[4,37,88]$ and found $\mathrm{PCL}=350 \mathrm{~ms}$ to be the most suitable for our rabbit ventricular model. Further, with this PCL both the number of APs that can be simulated and the duration of the refractory period following one membrane depolarisation are optimised. This means that enough APs are produced for a meaningful analysis and they are still spaced in a way that no overlap with the refractory period takes place. The total simulation time was fixed to $5250 \mathrm{~ms}$ and thus allows to cut off the first $1500 \mathrm{~ms}$ of transient time.

The so-called spark simulations were performed on four quarter Z-discs and are characterised by the lack of stimuli. Thus no action potential is triggered and the cell is left to evolve in time and is only subject to the stochastic processes that describe channel gating (see section 2.2.4 for details). The simulation time is constrained to $2500 \mathrm{~ms}$ and, compared to the one of AP simulations, sufficient for the observation of the quantities of interest. The long transient time during AP simulations is needed to allow for a "stabilisation" of the calcium concentrations in SR and cytosol, while for spark simulations this is not necessary, since the ion concentrations in the different compartments start in a steady state situation. Sparks, i.e. individual and spontaneous calcium release events at CRUs, are observed in experiments, but are often hard to quantify [89]. It is thus interesting to perform spark simulations to better understand spontaneous calcium release from the SR.

The two simulations types also define different quantities of interest. Unsurprisingly the AP simulations have their focus on action potentials and the calcium concentrations that emerge during the common ECC. In spark simulations on the other hand we concentrate much more on sparks and quarks as single events (these are described in detail below). The choice of four quarter Z-discs instead of one full Z-disc was made to seek for a possible calcium diffusion in z-direction and study potentially arising calcium waves $[40,63,90,91]$. We did not observe any proper calcium waves with the current setup and input parameters, merely so called macro sparks, i.e. sparks 
involving several CRUs along all three spacial directions where the usage of stacked Z-discs would be required.

\section{Quarks and Sparks}

Definitions of quarks and sparks are sometimes ambiguous, due to the fact, that often only a fistful of RyRs among hundreds is involved in the process and not rarely for very short time periods (some few milliseconds). We decided to adopt a definition of calcium quarks and sparks similar to the one proposed by Lederer and coauthors $[89,92,93]$. We set a minimum time for an event to be called either quark or spark to $20 \mathrm{~ms}$. The events are defined as follows: one and only one open RyR in one cleft represents a quark, while a spark is determined by at least two open RyRs in the same cleft.

These requirements allow us to discern between those events in which only one $\mathrm{RyR}$ is open for a longer period of time, i.e. a quark, and those in which one open RyR might trigger the opening of its neighbours in the following milliseconds, thus leading to a spark.

The definitions chosen for this study allowed us to pin down the events with notable changes in the calcium concentration as well, since these are directly related to the occurrence of sparks.

\subsubsection{Biomarkers}

The term biomarker has its origin in one publication by Britton et al. [54] and was used thereafter in further analysis of characteristics of cardiomyocytes [52, 94]. Particularly biomarkers represent some common characteristics of membrane potential, intracellular calcium concentrations and structural properties as e.g. number of open channels in a given cleft at a given time point.

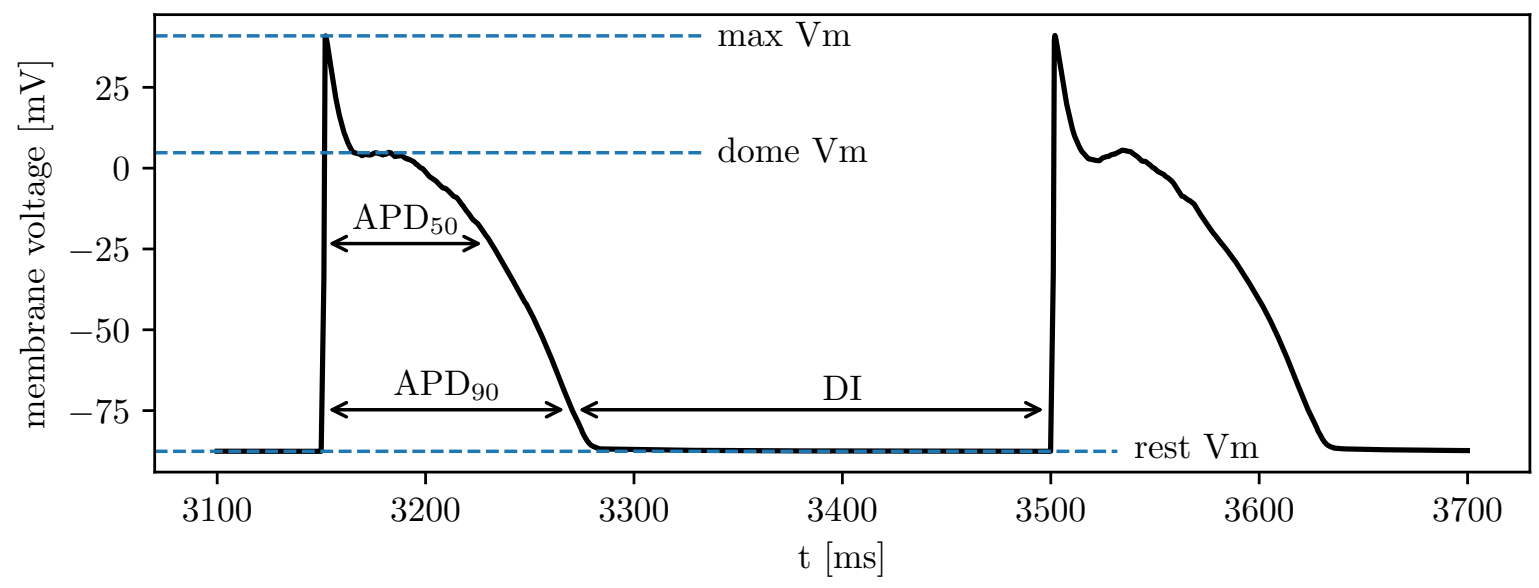

Figure 3.1 - Representation of the membrane voltage $V_{m}$ showing two action potentials and their characteristic biomarkers $A P D_{50}, A P D_{90}$, diastolic interval (DI), peak value, dome value and resting value of the $A P$. 
In figure 3.1 an illustration of several biomarkers from AP simulations is shown. There the action potential duration at $50 \%$ and $90 \%$ of the maximal value of the AP are depicted as $\mathrm{APD}_{50}$ and $\mathrm{APD}_{90}$, respectively. I also depicted the values of resting voltage, rest $\mathrm{Vm}$, dome membrane potential, dome $\mathrm{Vm}$, and peak value of the AP, max Vm together with the length of the diastolic interval, DI. These are examples of characteristic biomarkers of AP simulations.

Since biomarkers are often measured at one specific event, e.g. an AP or a calcium spark, the final value for a given biomarker is represented by the mean over all considered events.

In the case of AP simulations the complete list of computed biomarkers is the following: maximum and resting membrane potential, maximum positive membrane voltage increase, dome value of the membrane potential, action potential duration at $50 \%$ and $90 \%$, peak free and Fluo- 4 bound calcium concentrations, minimum free and Fluo- 4 bound calcium concentrations during diastole, time to peak of free and experimental calcium and sodium concentration.

For the spark simulations the list of computed biomarkers is the following: number of quarks and sparks as well as their ratio, peak free and Fluo-4 bound calcium concentrations, number of open RyRs per millisecond, full duration both at $50 \%$ (half maximum) and $90 \%$ of the maximal free and Fluo- 4 bound calcium concentration during sparks. The full duration at half maximum we often abbreviate to FDHM.

The values for the peak calcium concentrations are computed during the calcium transient, i.e. the calcium release event, for AP simulations and on single calcium sparks for spark simulations. In figure 3.2, which is part of figure 3 of [50], an example of a calcium transient is depicted. The calcium concentration as a function of time for both free and Fluo-4 bound calcium ions is shown. The maximum of this curve represents the biomarker for peak calcium concentration.

The choice of the biomarker is based on comparability with values from literature. While for AP simulations the choice of the biomarkers is straight forward considering the simplicity with which action potentials can be evaluated, this is not true for the spark simulations. There the process of biomarker selection was accompanied by considerations on how

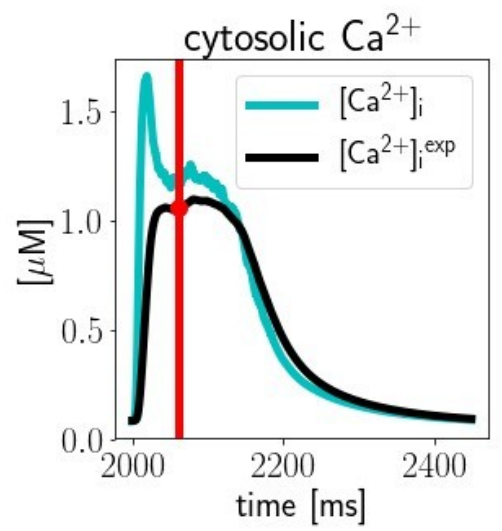

Figure 3.2 - Calcium concentration profiles in the cytosol of free and Fluo-4 bound ions during one transient in cyan and black, respectively. The graph is part of figure 3 of [50], in which a snapshot of the ECC in one full $Z$-disc is shown at the time point highlighted by the red bar, which can be ignored here. Adapted from [50], Creative Commons, CC BY-NC-ND 4.0. much its definition is describing the whole cell dynamics, since single sparks can be considered to be rare events. This means that a spark at a given CRU often occurs only once or twice per simulation (unlike action potentials that are periodic) and, since most of the clefts differ from each other, its characteristics 
can vary from CRU to CRU. Nevertheless, we found the peak values of calcium sparks as well as measures of their duration to be well suited to describe calcium dynamics in resting cardiomyocytes.

Note that the Fluo-4 output for spark simulations was implemented at a later point during the study (since it was not present in the model's original outputs). This means that the number of biomarkers considered for FDHM and peak Fluo-4 bound calcium is lower than the total amount of performed spark simulations. Nevertheless, as will be shown later in section 3.3.2, this amount suffices for an analysis with the polynomial chaos expansion with polynomial order up to 3.

\subsubsection{Parameter Input}

Cleftdyn allows for several input parameters that can be varied arbitrarily. The choice which parameters to vary to study their effect on the cardiomyocyte behaviour was based on the desire to focus on the effects cleft structures have on the cell dynamic. We selected the opening and closing rates of RyRs $k_{\text {plus }}$ and $k_{\text {close }}$, respectively, the permeabilities of RyR and LC channels $g_{\mathrm{RyR}}$ and $g_{\mathrm{LCC}}$, respectively, and the fixed strength of SERCA uptake $V p_{\max }$. These parameters but the last one are directly related to the dynamics inside each dyadic cleft (see section 2.2.4 for details). We chose to include the strength of SERCA uptake as well, since it is indirectly related to the cleft dynamics via the regulation of the intra-SR calcium concentration and is furthermore one of the parameters that best can be compared with the upregulation of SR calcium via common drug delivery $[5,19,68]$.

We thus selected $k_{\text {plus }}, k_{\text {close }}, g_{\mathrm{RyR}}, g_{\mathrm{LCC}}$ and $V p_{\max }$ as the parameters which would affect the intra-cellular dynamics most when introducing structural changes to the model, i.e. the new placement method for RyRs (see section 2.1.3).

It is clear that the impact of the LCC permeability $g_{\mathrm{LCC}}$ plays almost no role in spark simulations, since they are not stimulated and thus no activation of the LCCs due to the membrane potential can be observed. Nevertheless we included this parameter also for the spark simulations for two reasons: first the LCCs are subject to stochasticity and might eventually release calcium into the dyadic cleft proportional to the permeability, second we wanted to keep the two simulation methods (AP and spark) as comparable as possible among each other.

\section{Physiological Parameter Variations}

The choice of the varied parameter ranges was taken considering the corresponding values of previous studies $[23,35,44-46]$. The five selected parameters $k_{\text {plus }}, k_{\text {plus }}, k_{\text {close }}$, $g_{\mathrm{RyR}}, g_{\mathrm{LCC}}$ and $V p_{\max }$ are varied in the region bounded by the values listed in table 3.1. The choice of the parameter region is motivated by the need to cover physiological ranges and beyond.

In more detail; since the mentioned parameters have no direct physiological correspondent, it is essential to vary these in an interval with suited width. This ensures an output that lies in a physiological relevant region, since pathological biomarker values 


\begin{tabular}{l|c|c}
\hline Parameter & $\min$ & $\max$ \\
\hline$k_{\text {plus }}\left[\mathrm{ms}^{-1}{\left.\mu \mathrm{M}^{-\eta}\right]}\right.$ & $5.0 \times 10^{-5}$ & $5.0 \times 10^{-4}$ \\
$k_{\text {close }}\left[\mathrm{ms}^{-1}\right]$ & 0.1 & 1.0 \\
$g_{\text {RyR }}\left[\mathrm{mm}^{3} / \mathrm{s}\right]$ & $3.0 \times 10^{-4}$ & $3.0 \times 10^{-3}$ \\
$g_{\mathrm{LCC}}\left[\mathrm{\mu m}^{3} / \mathrm{s}\right]$ & $4.5 \times 10^{-4}$ & $4.5 \times 10^{-3}$ \\
$V p_{\max }[\mu \mathrm{M} / \mathrm{ms}]$ & 0.15 & 1.5 \\
\hline
\end{tabular}

Table 3.1 - Bounds in which the input parameter values of $k_{\mathrm{plus}}, k_{\mathrm{close}}, g_{\mathrm{RyR}}, g_{\mathrm{LCC}}$ and $V p_{\max }$ are varied.

have to be considered as well. Comparison to previous studies [35, 47] showed that the chosen ranges satisfy this requirement.

\section{Latin Hypercube Sampling}

The high computational costs of cleftdyn allow to produce only a small number of simulation runs as already mentioned. For a reduced number of samples in a higher dimensional parameter space a sampling method known as Latin hypercube sampling is strongly beneficial. In the following I will outline why this is the case.

Latin hypercube sampling draws its name from the

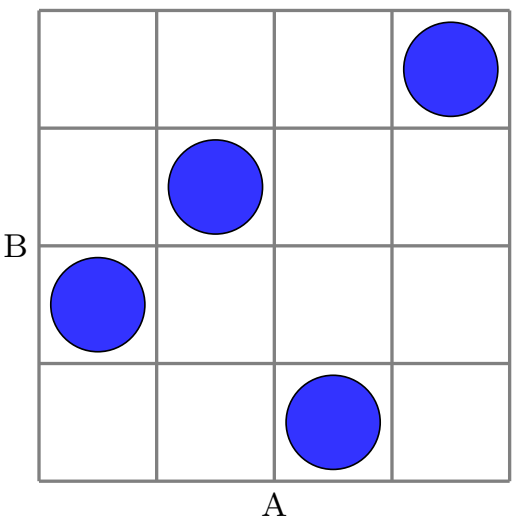

Figure 3.3 - An example of a Latin hypercube in two dimensions with parameters $A$ and $B$. The blue circles represent the samples drawn in parameter space. concept of a Latin square [95] as sketched in figure 3.3. This discrete square has only one entry for each row and each column. In the sketch this is shown for two example parameters A and B and for four entries depicted as blue circles. The Latin hypercube is the N-dimensional generalisation of this concept.

Sampling using a Latin hypercube requires to determine the number of samples $M$ prior the actual parameter determination. Each parameter or variable of the $\mathrm{N}$-dimensional hypercube is then subdivided into $\mathrm{M}$ intervals. Each sample that is drawn has knowledge of the previously drawn ones. Latin hypercube sampling thus allows for a creation of random numbers in a parameter or variable set ensuring that the full parameter ranges are covered as well as possible with only few samples. Mere uniform random number generation is in contrast responsible for a creation of a set of values that are totally uncorrelated.

\subsubsection{Sampling Action Potentials and Sparks}

The population of simulations from the varied parameters $k_{\text {plus }}, k_{\text {close }}, g_{\mathrm{RyR}}, g_{\mathrm{LCC}}$ and $V p_{\max }$ is analysed and for each population member the biomarkers described in 3.1.2 
are determined. Every simulation yields one biomarker value, usually the average of several quantities as e.g. the mean of $\mathrm{APD}_{90}$ from many APs is computed.

\section{Sampling Action Potentials}

In figure 3.4 two biomarkers from the $\mathrm{AP}$ simulations, $\mathrm{APD}_{90}$ and peak calcium concentration $\left[\mathrm{Ca}^{2+}\right]$, are plotted for a population of 281 action potential simulations versus the five simultaneously varied parameters $k_{\text {plus }}, k_{\text {close }}, g_{\mathrm{RyR}}, g_{\mathrm{LCC}}$ and $V p_{\max }$.
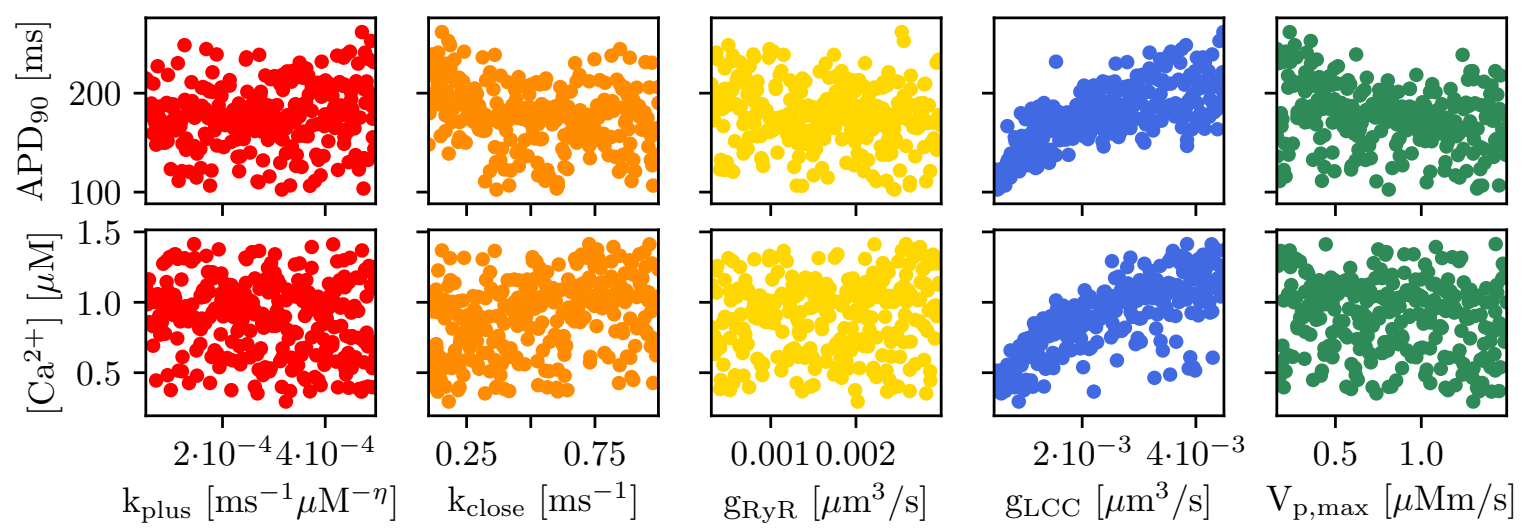

Figure 3.4 - Examples of two biomarkers from action potential simulations, $A P D_{90}$ and mean peak calcium concentration, as function of five varied parameters $k_{\text {plus }}, k_{\text {close }}$, $g_{L C C}, g_{R y R}$ and $V p_{\max }$.

For each biomarker fluctuations can be observed. This is due to two major facts. First: the parameters are all varied at the same time leading to a spread output in the biomarkers. Second: the stochasticity of cleftdyn causes inherently fluctuations in the output.

The projections of biomarker values onto the parameter planes show weak trends. The impossibility of depicting the full six dimensional data set hampers the possibility of directly recognising dependencies on the considered parameters.

Nevertheless the trend of some biomarkers with respect to given parameters, e.g. here $\mathrm{APD}_{90}$ versus LCC permeability $g_{L C C}$, is apparent already from the projection onto the parameter axis and thus motivates a quantitative sensitivity analysis. This relationship is not very surprising when considering the mechanism that triggers the excitation contraction coupling. The opening of the LCCs is responsible for an influx of calcium that allows the opening of RyRs. Tuning the permeability of the LCCs towards higher values increases the influx of calcium and thus enhances this effect, leading to a higher probability for the RyRs to open and indirectly to a higher calcium concentration. The higher intracellular calcium in turn alters the ionic equilibrium in the cardiomyocyte affecting the membrane potential as well prolonging its duration.

Note that the effect is clearly visible in the one dimensional projection though all parameters are varied simultaneously, meaning that the impact of $g_{L C C}$ on the considered biomarkers is considerably high. 


\section{Sampling Sparks}

Figure 3.5 shows an example of two biomarkers, namely FDHM and peak Fluo-4 bound calcium as function of the varied parameters $k_{\text {plus }}, k_{\text {close }}, g_{\mathrm{RyR}}, g_{\mathrm{LCC}}$ and $V p_{\max }$. Since again, like for the AP biomarkers, a representation in five dimensions is not feasible, I show the one-dimensional projections on the single varied parameter ranges.
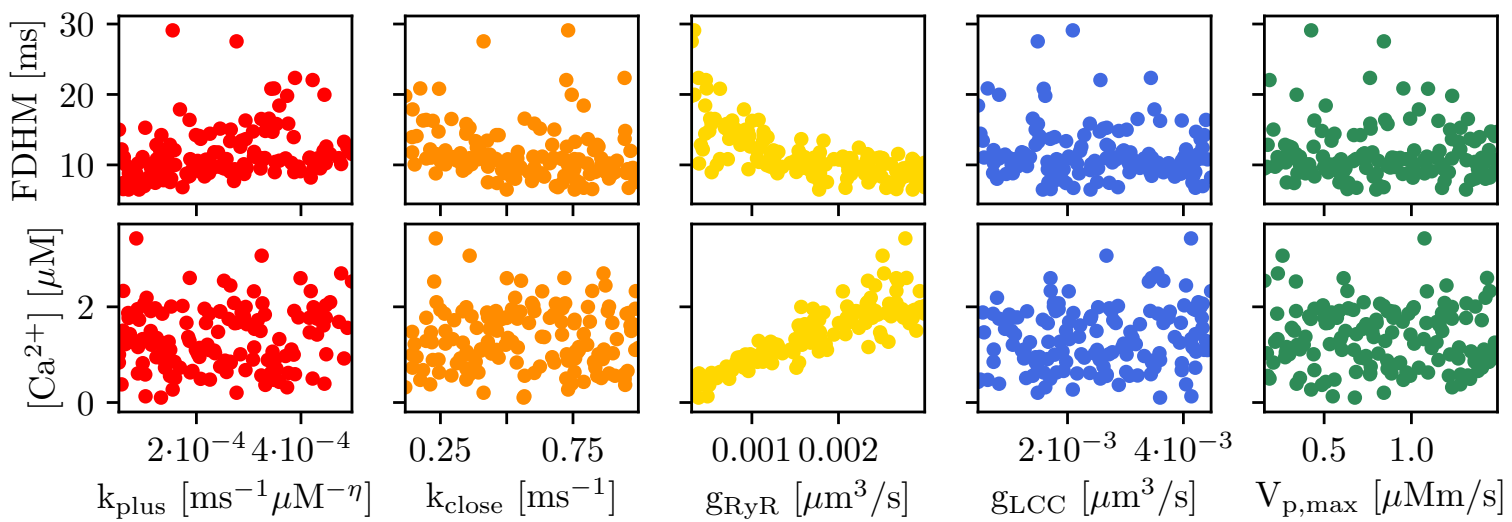

Figure 3.5 - Examples of two biomarkers from spark simulations, FDHM and mean peak calcium concentration, as function of the five varied parameters $k_{p l u s}, k_{c l o s e}, g_{L C C}$, $g_{R y R}$ and $V p_{\max }$.

At first glance the dependencies of the shown biomarkers on the considered parameters reveals a strong influence of the RyR permeability $g_{R y R}$ on the calcium peak concentration. This evident trend is visible despite the simultaneous variation of the other parameters pointing out the strength of this impact. The influence of $g_{R y R}$ on peak calcium shown in figure 3.5 is clear and immediately relatable to the quantity of calcium RyRs allow to flow from the SR into the cytosol.

Another trend is visible here, the decay in the FDHM with respect to the RyR permeability. The reason for this is not immediately clear, but becomes so when considering again the amount of calcium extruded from the SR. The higher this amount is, the steeper the gradient inside each single cleft is, resulting into a quicker activation of neighbouring and still inactive RyRs. A fast channel activation leads to a premature closing of the RyRs, too, and thus to a shortened FDHM.

Both apparent dependencies motivate a deeper analysis of the relation between parameters and biomarkers.

\subsection{Parameter and Biomarker Estimation}

We adopted several strategies to asses the biomarkers and parameters relevant for further analysis. Based on the goal to first find a parameter region and a set that would generate physiological output, we employed linear sensitivity analysis to asses the most relevant biomarkers and the related parameters with the greatest impact on the output. 


\subsubsection{Linear Sensitivity Analysis and Sobol Coefficients}

In order to understand the impact certain parameters have on the system's output, it is helpful to consider a linear regression fit to the biomarkers first. We employed Bayesian linear regression [96] using the Python libraries and toolboxes Edward [97] and TensorFlow [98]. We further employed the linear regression function of the Python library scikit learn [99]. The linear regression allows to consider the linear sensitivity as an indicator for the impact parameters have on the analysed data.

The linear sensitivity $s_{X Y}$ of a biomarker $Y$ with respect to a given parameter $X$ is defined through the slope $S_{X Y}$ of the linear regression fit, $Y=C+S_{X Y} X$, via:

$$
s_{X Y}=\frac{X_{\text {ref }}}{Y_{\text {ref }}} S_{X Y}
$$

where $X_{\text {ref }}$ and $Y_{\text {ref }}$ are chosen reference parameter-biomarker values that define the local slope coefficient of the sampled data. Thus the sensitivity can be considered to be the normalisation of the linear regression slope at a chosen point in parameter space.

Further we also computed the so called Sobol coefficients of all computed biomarkers [100-102]. The Sobol coefficient of a given biomarker with respect to a parameter $X$ is computed as the ratio of the variance of that biomarker $D_{X}$ and the sum of all variances $D=\sum_{X} D_{X}$ :

$$
S_{X}=\frac{D_{X}}{D}
$$

The set of Sobol indices or coefficients $\left\{S_{X}\right\}$ sums up to one, such that the impact of each single parameter is related to the one of the others. The Sobol indices can be considered as a global measure for the impact some input parameters or variables $\boldsymbol{X}$ have on the output. We computed the Sobol coefficients using the function obtained from the main regression method we employed, the polynomial chaos expansion (detailed in section 3.3).

Since the Sobol coefficients only reveal the impact of parameters on the output relative to other parameters and we were interested in selecting the biomarkers relevant for the study as well, we employed them only to double check the choice of parameters made through linear sensitivity analysis. Phrased differently; the Sobol indices neglect the global impact that input parameters can have on output biomarkers, while the sensitivity analysis allows to asses the overall effect as well. Sobol coefficients carry the relative information between different parameters, while the linear sensitivity analysis captures the absolute impact on biomarkers, too.

Both methods were applied to select relevant biomarkers. Out of many considered biomarkers (of which most are listed in tables 2 and 3 of [50] and sections 3.1.2 and 4.1) seven are of particular interest. These biomarkers are: $\mathrm{APD}_{90}$, systolic peak calcium and diastolic calcium in the case of action potential simulations and full duration at half maximum, peak $\left[\mathrm{Ca}^{2+}\right]$ both of calcium sparks, spark rate and quark to spark 
ratio in the case of spark simulations.

These biomarkers turned out to be particularly affected by parameter variations. Further biomarkers that showed similar behaviour and could be related to the one mentioned here were discarded. For example $\mathrm{APD}_{50}$ was not considered, since it can be easily related to $\mathrm{APD}_{90}$. As another example the number of quarks is linked through the quark to spark ratio to the number of measured sparks. In the following I will refer to the seven named biomarkers as selected biomarkers. Later during the study the time to peak of calcium concentration during an action potential became interesting and relevant for AP simulations as well. This biomarker is considered in section 3.4.2 and in figures 8 and 10 of [50].

Since the linear sensitivity can be computed at arbitrary points in space we had to choose a particular one. First we picked the median value of the varied parameter range, but changed this later to the parameter set that was selected after a parameter sieve and a polynomial chaos expansion regression were applied (see sections 3.2.2 and 3.3.2 for details). This shift did partially affect the strength of the sensitivities, but not their overall trend, which is the quantity of interest here.

\section{Linear sensitivities of AP and spark simulations}

The linear sensitivity $s_{X Y}$ of action potential and spark simulation is plotted for the selected biomarkers in figures 4 and 6 of [50], which I depict here again for convenience as figures $3.6 \mathrm{a}$ and $3.6 \mathrm{~b}$, respectively. In both figures the sensitivities are depicted versus the five varied parameters $k_{\text {plus }}, k_{\text {close }}, g_{\mathrm{RyR}}, g_{\mathrm{LCC}}$ and $V p_{\max }$ and colour-grouped by the considered biomarkers.

Figure 3.6a shows how strong the positive impact of LCC permeability $g_{\mathrm{LCC}}$ is on all three action potential biomarkers. The calcium concentration values clearly are positively affected by the strength of the calcium influx from the exterior of the cell, but also the duration of APs is affected. This last point can be understood when considering the amount of calcium that is delivered into the dyadic cleft every single AP. Increasing this means that the RyRs in turn have a higher chance to open and thus prolong the duration of the action potential. This is in line with the results presented in [54]. Counterintuitive at a first glance is the negative effect an increase of $k_{\text {plus }}$ has on the calcium concentration, but in this case the APD is slightly prolonged causing more ions to be extruded from the SR and thus a reduced peak value of the calcium concentrations. For $k_{\text {close }}$ the opposite is true. A similar effect occurs for the RyR permeability, where an increase in $g_{\mathrm{RyR}}$ values is followed by a decrease in the diastolic calcium concentration; this time with the difference of increasing peak calcium and a hampered APD. The former effect is directly related to the stronger calcium release through RyRs. Finally an increasing SERCA pump strength results in a decrease in all three observed biomarkers. The action of SERCA is immediate and thus calcium is pumped back into the SR faster than the timescale on which the values of peak calcium and especially diastolic calcium can be settled.

Regarding the spark simulations figure $3.6 \mathrm{~b}$ gives insight into the immediate consequences of parameter variations onto the selected biomarkers. Unsurprisingly the 


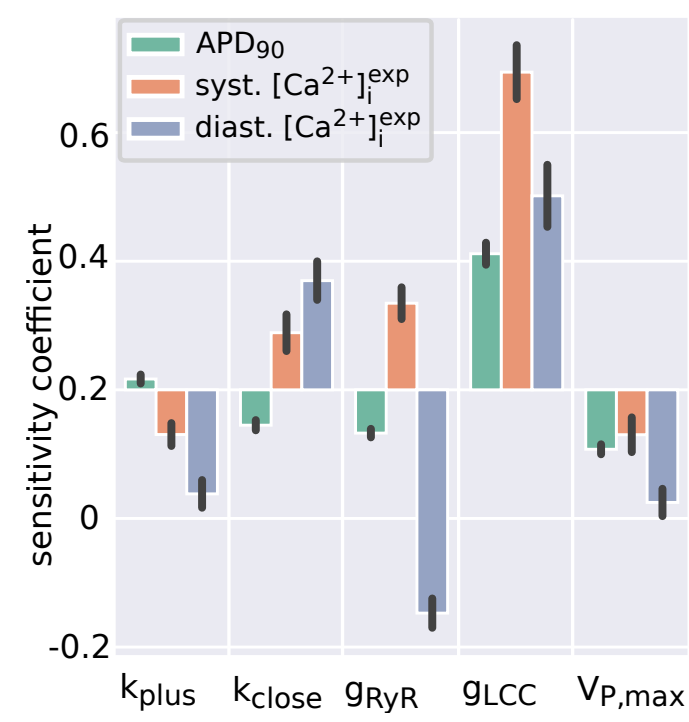

(a) - Linear sensitivity coefficients of $A P D_{90}$, peak systolic calcium and diastolic calcium concentrations for the input parameters $k_{\mathrm{plus}}$, $k_{\text {close }}, g_{\mathrm{RyR}}, g_{\mathrm{LCC}}$ and $V p_{\max }$.

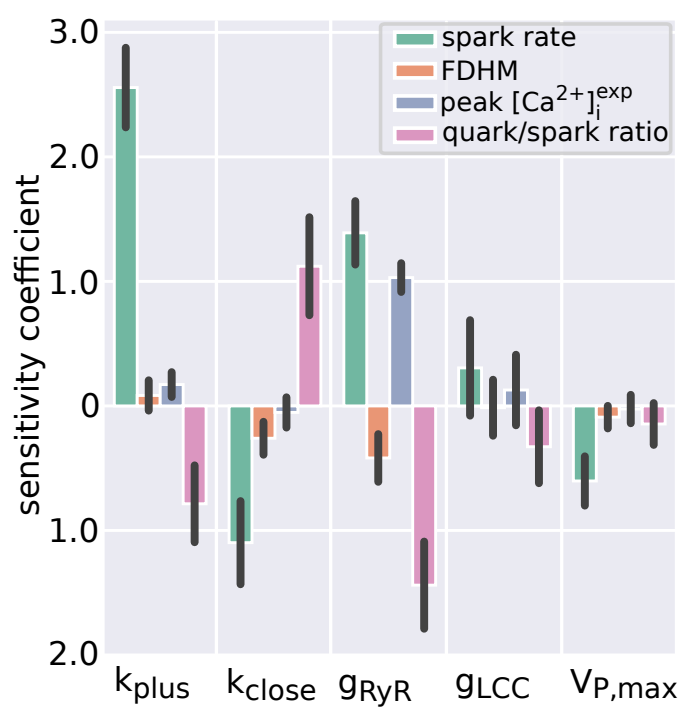

(b) - Linear sensitivity coefficients of spark rate, FDHM, peak calcium concentration and quark to spark ratio for the input parameters $k_{\text {plus }}, k_{\text {close }}, g_{\mathrm{RyR}}, g_{\mathrm{LCC}}$ and $V p_{\max }$.

Figure 3.6 - Coefficients from the linear sensitivity analysis for different AP and spark simulation biomarkers as functions of the selected input parameters $k_{\text {plus }}, k_{\text {close }}, g_{\mathrm{RyR}}$, $g_{\mathrm{LCC}}$ and $V p_{\max }$. Reprinted from [50], Creative Commons, CC BY-NC-ND 4.0.

LCC permeability plays barely a role in spark simulations, while the opening and closing rates of RyRs are crucial for the observed number of sparks and quarks and whenever spark rates increase the quark to spark ratio decreases. $k_{\text {plus }}$ promotes the spark rate as well as $g_{\text {RyR }}$, while for $k_{\text {close }}$ the opposite is true. Again without surprise it can be observed that the values of the peak calcium are enhanced by stronger RyR permeabilities. The full duration at half maximum instead is only affected negatively in a minor manner by the closing rate and the permeability of RyRs. Finally the SERCA pump strength only has an effect on the spark rate, meaning that a fast refilling SR will affect only the amount of observed sparks.

The linear sensitivities give valuable insight into the general trends of biomarkers with respect to the input parameters and were used to ultimately asses which output was most sensitive upon parameter variations, but in order to confine the parameter region to one that would deliver physiological biomarker values, further analysis has to be performed.

\subsubsection{Biomarker Sieve}

To asses the ranges of parameters that yield physiological output I chose to sieve the biomarker values using the values provided in literature [13, 68, 88, 94, 103, 104]. The selected values are listed in table 3.2 and in tables 2 and 3 from [50] and in section 4.1. During the screening I consider the biomarker values and filter them with the 
considered limiting values from literature. The resulting simulations then reveal the first rough parameter ranges for which the output of the simulations can be considered to be physiological.

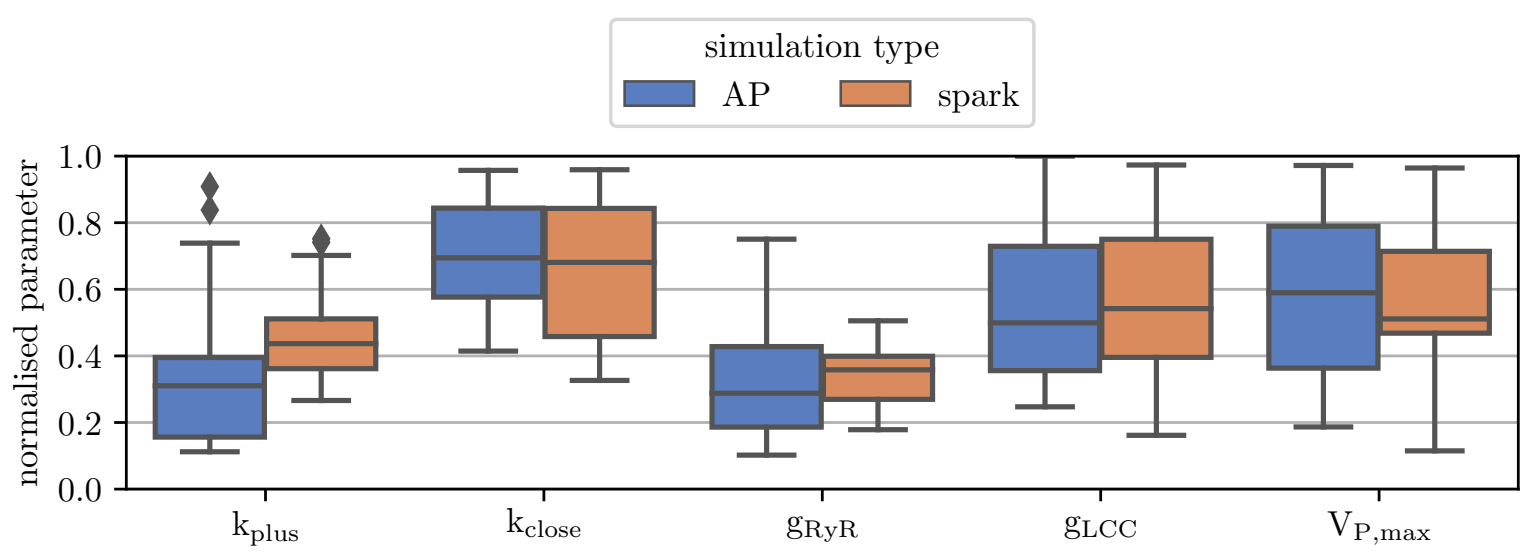

Figure 3.7 - Boxplot showing the normalised parameters sieved with the biomarker limits for two different simulation approaches, i.e. the action potential and the spark simulations in blue and orange, respectively. The ranges show outliers only for three values of $k_{\text {plus }}$, while all other parameters lie in the whisker limits. Table 3.2 lists the maximum and minimum of the not normalised parameters according to the simulation type.

Because of the different orders of magnitude and units describing them and to best illustrate their ranges, the parameters are normalised to unity employing the maximum parameter value. In this picture 1 is the maximal value a parameter can assume, while 0.1 represents the lower parameter limit. To give an example, a value of the LCC permeability $g_{\mathrm{LCC}}$ of 1 corresponds to the maximum of the sampling interval, i.e. $4.5 \times 10^{-3} \mu \mathrm{m} / \mathrm{s}^{3}$.

The normalisation of the parameters has the advantage of making the parameter ranges that lead to physiological output comparable. Figure 3.7 shows the ranges of the varied parameters for the two main simulation procedures action potential and spark in blue and orange, respectively.

The pairwise overlap of the ranges is a good indicator for the consistency of cleftdyn simulations regardless of the simulation type. Further on, an overlap of the ranges of all parameters can be observed, which confirms the choice of the initial intervals.

The maximum and minimum values of the such sieved parameter intervals are listed for both simulation approaches in table 3.2. These values are the limiting maximal ranges depicted in figure 3.7. Attention has to be paid comparing these two values, since the ranges shown in the figure are normalised, while the table lists the original values.

Of the 281 performed AP simulations 23 were positively sieved out and of the 297 spark simulations a total of 16 passed the filtering. 


\begin{tabular}{l|cc|cc}
\hline Parameter & \multicolumn{2}{|c|}{ AP } & \multicolumn{2}{c}{ spark } \\
\hline & min. & max. & min. & $\max$. \\
$k_{\text {plus }}\left[\mathrm{ms}^{-1}{\left.\mu \mathrm{M}^{-\eta}\right] \times 10^{-4}}\right.$ & 0.56 & 4.5 & 1.3 & 3.8 \\
$k_{\text {close }}\left[\mathrm{ms}^{-1}\right]$ & 0.41 & 0.95 & 0.33 & 0.96 \\
$g_{\text {RyR }}\left[\mu^{3} / \mathrm{s}\right] \times 10^{-3}$ & 0.3 & 2.3 & 0.5 & 1.5 \\
$g_{\mathrm{LCC}}\left[\mu^{3} / \mathrm{s}\right] \times 10^{-3}$ & 1.1 & 4.5 & 0.7 & 4.4 \\
$V p_{\max }[\mu \mathrm{M} / \mathrm{ms}]$ & 0.28 & 1.5 & 0.17 & 1.4 \\
\hline
\end{tabular}

Table 3.2 - Parameter values of the range limits of the sieved data for the two simulation approaches action potential and spark. The values can be related to the limiting values of the ranges shown in figure 3.7 .

The ranges found here allow to confine the quest for a parameter set that yields physiological output. This was undertaken with the aid of the regression method via polynomial chaos expansion.

\subsection{Polynomial Chaos Expansion}

The following section focuses on the theory of the polynomial chaos expansion as well as its application in the present study. Polynomial chaos expansion (PCE) is a regression method that allows to approximate some given output $Y$ with a function of a given input $X$. In this work we employed the PCE for the construction of a function or surrogate model that approximates a model that cannot be simulated often enough to allow a statistical interpretation as it is done for example when using Monte Carlo like simulations. The simulations done with cleftdyn to asses the impact the varied parameters have on the model output represent exactly this case.

PCE is based on an approximation of multivariate and orthonormal polynomials as for example Hermite or Legendre polynomials.

\subsubsection{Polynomial Chaos Expansion - Theory}

The description of the basic ideas behind the PCE are derived mainly from $[56,58$, 105-107]. Given a computational model $\mathcal{M}$ with an $M$-dimensional vector $\boldsymbol{X}$, which contains uncertain or random input parameters (in our case $k_{\text {plus }}, k_{\text {close }}, g_{\mathrm{RyR}}, g_{\mathrm{LCC}}$ and $V p_{\max }$, so $M=5$ ) we can consider a joint probability density function (PDF) $f_{\boldsymbol{X}}$ that describes the distribution of the model's input values, $\boldsymbol{X}$. For an output quantity $Y=\mathcal{M}(\boldsymbol{X})$ (which in our case would be a biomarker) the polynomial chaos expansion is defined by:

$$
Y=\sum_{\omega \in \mathbb{N} M} w_{\omega} \Psi_{\omega}(\boldsymbol{X})
$$


with coefficients $w_{\omega}$ and $\Psi_{\omega}$ as the product of univariate polynomials $\pi_{\omega_{i}}^{(i)}$ with polynomial degree $\omega_{i}$ of the $i$-th parameter entry:

$$
\Psi_{\omega} \equiv \prod_{i=1}^{M} \pi_{\omega_{i}}^{(i)}\left(x_{i}\right)
$$

The $\pi_{k}^{(i)}$ have to fulfill orthonormality with respect to the probability density function of input parameters $f_{\boldsymbol{X}}$, in other words their scalar product has to yield the Kronecker delta:

$$
\left\langle\pi_{j}^{(i)}, \pi_{k}^{(i)}\right\rangle \equiv \int \pi_{j}^{(i)}(u) \pi_{k}^{(i)}(u) f_{X_{i}}(u) \mathrm{d} u=\delta_{j, k} .
$$

Polynomials that fulfill this requirement are classically Legendre and Hermite polynomials for uniform and Gaussian distributions, respectively [58].

In order to apply the PCE to any mathematical model, the expansion defined in equation (3.3) has to be truncated in some way. Often a maximal total polynomial degree $p$ is chosen such that the PCE is approximated by [56]:

$$
Y \approx \sum_{\omega \in \mathcal{A}} w_{\omega} \Psi_{\omega}(\boldsymbol{X})
$$

where the set $\mathcal{A}$ from which the polynomial degrees are drawn is defined as:

$$
\mathcal{A}=\left\{\omega: \sum_{i=1}^{M} \omega_{i} \leq p\right\}
$$

The computation of the approximated model function $(M)$ is therefore reduced to the determination of the coefficients $w_{\omega}$. This is done here using the so called LASSO method that uses the polynomial chaos basis $\Psi_{\omega}$ to minimize an ordinary least-square with a regularisation term given by the $L^{1}$-norm [108].

\subsubsection{Polynomial Chaos Expansion - Application}

In our study the PDF $f_{\boldsymbol{X}}$ of input parameters is a uniform distribution, thus Legendre polynomials are best suited as candidates for the polynomial chaos expansion. In this study we employed the Python library Chaospy [109] for the determination of the orthonormal basis of polynomials. In order to approximate the model of the biomarker outputs with a truncated polynomial chaos expansion, it is necessary to determine the coefficients of the expansion itself (see equations (3.3) and (3.6)).

The method for the coefficient determination can be chosen among several, for instance least-square minimization [110], LASSO or Ridge regression [108, 111]. We 
decided to opt for the $L A S S O$ (least absolute shrinkage and selection operator) method. The LASSO method minimizes the following expression:

$$
g=\arg \min _{w_{\omega}} \frac{1}{n} \sum_{i=1}^{n}\left(y_{i}-\sum_{\omega \in \mathcal{A}} w_{\omega} p_{\omega}\left(x_{i}\right)\right)^{2}+\alpha \sum_{\omega \in \mathcal{A}}\left|w_{\omega}\right|
$$

with respect to the coefficients $w_{\omega}$, with $n$ samples, with $y_{i}$ as the output biomarker and $p_{\omega}\left(x_{i}\right)$ as the polynomial chaos basis with input parameters $x_{i}$. The second sum is the regularisation term representing the $L^{1}$-norm multiplied by a penalisation factor $\alpha$.

The regularisation term allows with the penalisation factor $\alpha$ to reduce the number of coefficients $w_{\omega}$ to be determined. This happens when the hypercube spanned by $L^{1}$-norm in coefficient space and the minimised function intersect on an axis forcing some $w_{\omega}$ to zero. This is schematically represented for the two dimensional case in figure 3.8, where it is shown how the intersection of the constraint and the $L^{1}$-norm enforces one parameter $\left(w_{1}\right.$ here) to be zero. A penalisation factor $\alpha=0$ reduces the LASSO to a least square method, in which all coefficients are considered. The LASSO regression used here is the one that can be found in the linear modelling module of the scikit-learn python library [99].

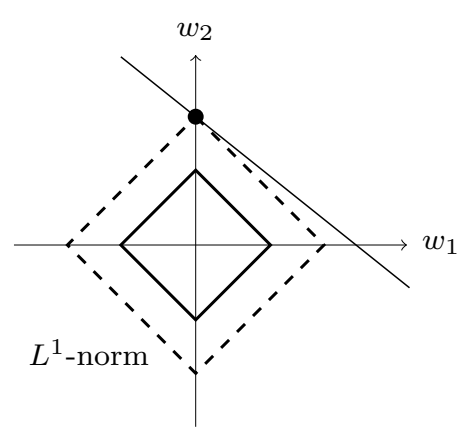

Figure 3.8 - Schematic graph of the regularisation with the $L^{1}$-norm as it happens for the LASSO regression. Depicted are two arbitrary coefficients $w_{1}$ and $w_{2}$ and the $L^{1}$-norm, on which the constraint shown as a straight line is regularised. additionally computed a cross validation score (CVS). This is calculated using the correspondent function of the scikit-learn python library [99]. The CVS compares the estimated function (in this case the LASSO regression) to the original data using a specific cross-validation splitting strategy, which we chose to be 10-fold. A $k$-fold splitting strategy divides the data set into $k$ consecutive subsets and uses each of them as a validation set for the remaining $k-1$ subsets that form the training set. In other words, the cross validation with $k$-fold splitting strategy represents the generalisation of the "leave one out" cross validation.

The CVS is computed using the $R^{2}$-score, which, for an index subset $\mathcal{I} \subseteq\{1, \ldots, N\}$ of the $N$ data points, is defined as:

$$
R^{2}=1-\frac{\sum_{i \in \mathcal{I}}\left(x_{i}-y_{i}\right)^{2}}{\sum_{i \in \mathcal{I}}\left(x_{i}-\langle x\rangle\right)^{2}}
$$

where $x$ and $\langle x\rangle$ are the original biomarker values and their mean, respectively, and $y$ are the predicted values from the PCE using only $x_{i}$ with $i \in\{1, \ldots, N\} \backslash \mathcal{I}$ as training data. Equation (3.9) shows that a CVS of 1 represents the best possible score, 
while a CVS of 0 stands for a regression that is as good as the mean value of the data. Negative scores denote a regression that performs worse than the mean $\langle x\rangle$. All cross validation scores presented from now on represent the average of the $R^{2}$-score of the subsets that are defined by the splitting with the $k$-fold method.

The computation for the truncated PCE (3.6) looks for the penalisation factor $\alpha \geq 0$, drawn from a predefined interval, that yields the best cross validation score for the LASSO regression and finally computes the approximating function with the optimised LASSO.

The cardinality $P$, finally, is the number of coefficients that have to be determined during the regression using both the maximum polynomial order $p$ and the total number of input parameters $M[56,107]$ :

$$
P=\left(\begin{array}{c}
M+p \\
p
\end{array}\right)
$$

With a specific set of data it is desirable to always have at least two to three times as many data points as the total number of coefficients to be determined in order to exclude overfitting in the first place.

In the following section 3.3.3 the application of the PCE regression onto the data set gained from AP and spark simulations is illustrated.

\subsubsection{Contours of a Response Function}

Once the PCE regression is applied to the selected biomarkers we get what will in the following be called response function and later also surrogate model. This function can help us, together with the bounds determined with the filtering of the initial data described in section 3.2.2 and in table 3.2, to further confine the parameter space to a region that yields physiological output.

Since the parameter space is five-dimensional and during the PCE regression no dimensionality reduction is applied, the response surface will be defined in six dimensions. To grasp the shape of the response function we decided to fix three parameters and to project it onto the plane spanned by the remaining two parameters producing a contour plot. The choice of the parameters that span this plane is based on the results from the linear sensitivity analysis (see figures 4 and 6 in [50] and section 3.2.1) and the fact that most of the relevant information can be drawn from those parameters with the strongest impact. As a consequence we chose $g_{\mathrm{LCC}}$ and $V_{\mathrm{p} \text {, max }}$ to span the projection plane for the regression surface from AP simulations and $k_{\text {plus }}$ and $g_{\text {RyR }}$ the one from the spark simulations. The remaining three values are then fixed at a give value, which later on we chose to be the selected parameter set.

The response surfaces obtained in this way are depicted in figures 5 and 7 in section 4.1 and in [50] and are discussed in detail ibidem.

For the determination of the so called selected parameter, for which all parameters yield physiological output for any considered biomarker set, it was necessary to employ 
the concomitant aid from the filtered parameter ranges (table 3.2) and the accepted ranges. In this way a set of parameters that lies in the white region of panel $\mathrm{D}$ in figures 5 and 7 in [50] for each considered parameter and for all six depicted biomarkers, both from AP as from spark simulations could be found.

\subsection{Impact of Structure Modifications}

The purpose of the present study is to asses the impact of modifications in the subcellular geometry, specifically in the location and placement of RyRs in dyadic clefts, on relevant output measures. In order to do so we first compared the state-of-the-art placement method [35] with the newly developed one from the Soeller group [1] (see section 2.1.3 for a detailed discussion on the state of the art regarding channel placement). Further we developed a measure that would allow us to analyse the properties and the effects of the cluster structure on the selected biomarkers.

\subsubsection{Regular and Soeller Placement}

As described in section 2.1.3 the authors of [1] introduced a placement algorithm which reproduces the geometrical structure of RyR clusters better than those previously used. They suggest to include this algorithm into new mathematical models capable of describing subdyadic structures.

Since cleftdyn employed a regular placement method for RyRs before [23, 24, 35, 43-47] as well, we decided to assess the importance of the modifications to the RyR cluster in the model itself. In order to do so we performed a series of 10 simulations for each protocol and for the two different placement methods. The regular placement has an equidistant spacing of $40 \mathrm{~nm}$, while for the Soeller placement we chose a mean distance between channels of again $40 \mathrm{~nm}$ with a standard deviation of $7.4 \mathrm{~nm}$ as it is outlined in [1]. All 40 simulations were performed with the selected parameter set in table 1 , fourth column of [50].

The resulting values of the biomarkers of interest are depicted in figures 8 and 9 of [50] and in section 4.1 for the action potential and spark simulations, respectively. The results are described and discussed ibidem. Note, in the case of the AP simulations we chose a further biomarker to be depicted, the time to peak of the calcium transient.

\subsubsection{Different Placement Measures}

To further understand the impact of the RyR cluster shape and structure on relevant biomarkers, we generated a population of simulation runs, in which, with the parameters $k_{\text {plus }}, k_{\text {close }}, g_{\mathrm{RyR}}, g_{\mathrm{LCC}}$ and $V p_{\text {max }}$ fixed at the suggested values, we varied two quantities involved in the generation of the Soeller placement. The mean of the exponential distribution $\langle$ distRyR $\rangle$, from which the distance to the next placed channel is drawn, is varied uniformly between 20 and $70 \mathrm{~nm}$, and its standard deviation distRyR $R_{\sigma}$ is varied 
between 0.1 and $50 \mathrm{~nm}$. Like for the input parameters $k_{\text {plus }}, k_{\text {close }}, g_{\mathrm{RyR}}, g_{\mathrm{LCC}}$ and $V p_{\max }$ a Latin square was generated for the two parameters.

We then performed $67 \mathrm{AP}$ and 96 spark simulations with varying 〈distRyR $\rangle$ and $\operatorname{distRyR} R_{\sigma}$. For each set of simulation runs we could compute several measures that characterise the RyR cluster. In this way we could understand how the geometrical properties of the RyR cluster itself would affect the output of cleftdyn.

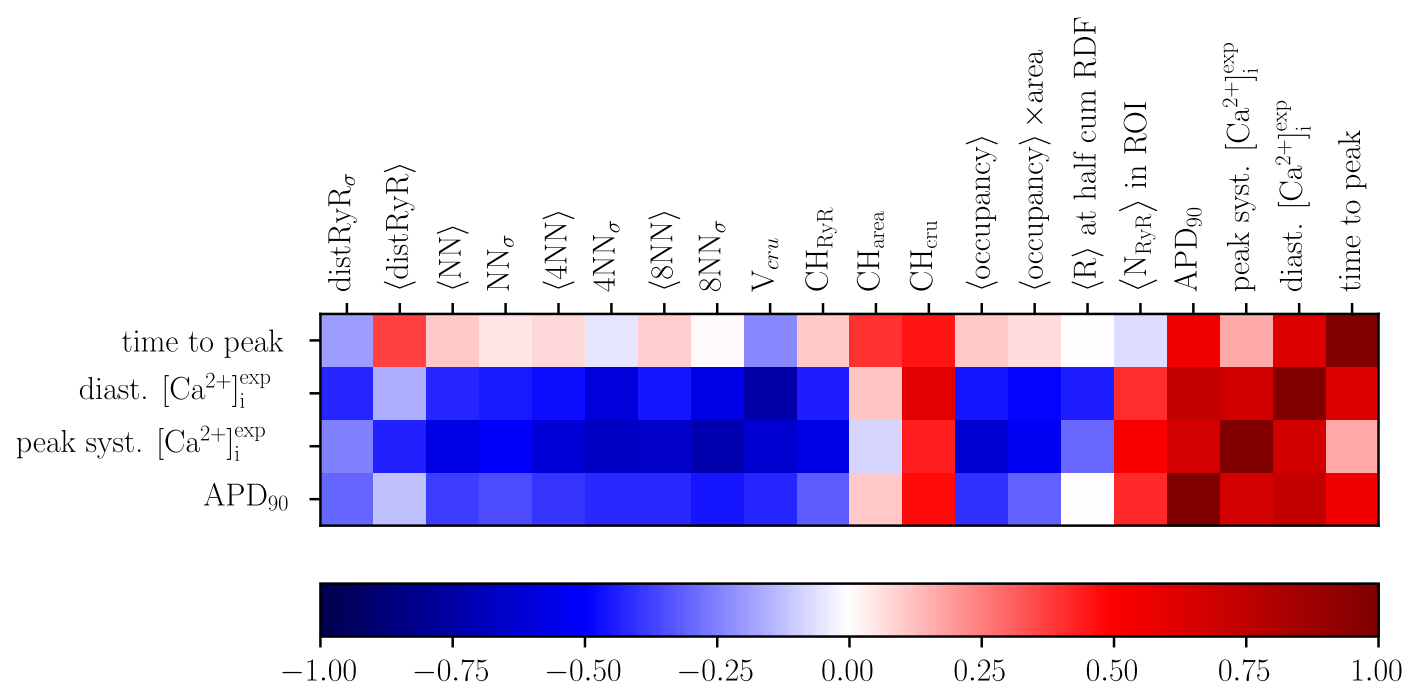

Figure 3.9 - Cross-correlation of four AP biomarkers (APD $D_{90}$, systolic and diastolic calcium concentration and time to peak) on the $y$-axis with measures for RyR cluster shape and structure on the $\mathrm{x}$-axis, where the biomarkers are listed again as well. One corresponds to full correlation, while -1 shows maximally negatively correlated measures.

Since the varied input parameters here just describe the mean and the standard deviation of the distribution generating the RyR realisations, they cannot be directly related to the actual shape or structure of the RyR cluster.

In order to classify the different shapes, densities and packing of the RyR cluster we employed several measures partly inspired and adopted from [112]: the mean $k$ nearest neighbour distance $\langle k \mathrm{NN}\rangle$ with $k \in\{1,4,8\}$ and its respective standard deviation $k \mathrm{NN}_{\sigma}$, the volume of the CRU $\mathrm{V}_{c r u}$, the convex hull normalised on number of RyRs, cleft area and $\mathrm{CRU}$ volume $\mathrm{CH}_{m}$ with $m \in\{\mathrm{RyR}$, area, cru $\}$ respectively, the mean occupancy 〈occupancy $\rangle$ and its product with the area, the mean number of RyRs in the region of interest $\left\langle\mathrm{N}_{\mathrm{RyR}}\right\rangle$ and the mean radius at half cumulative radial distribution function. How the convex hull, $\mathrm{CH}$, and the occupancy are computed is detailed below.

For each measure the results varied according to the number of samples produced (67 AP and 96 spark simulation runs). It was thus possible to calculate the Pearson correlation coefficient of the geometrical measures described above with the different biomarkers.

In figure 3.9 the correlation between the geometry measures and three main AP biomarkers plus time to peak of the peak calcium concentration are shown. A value 


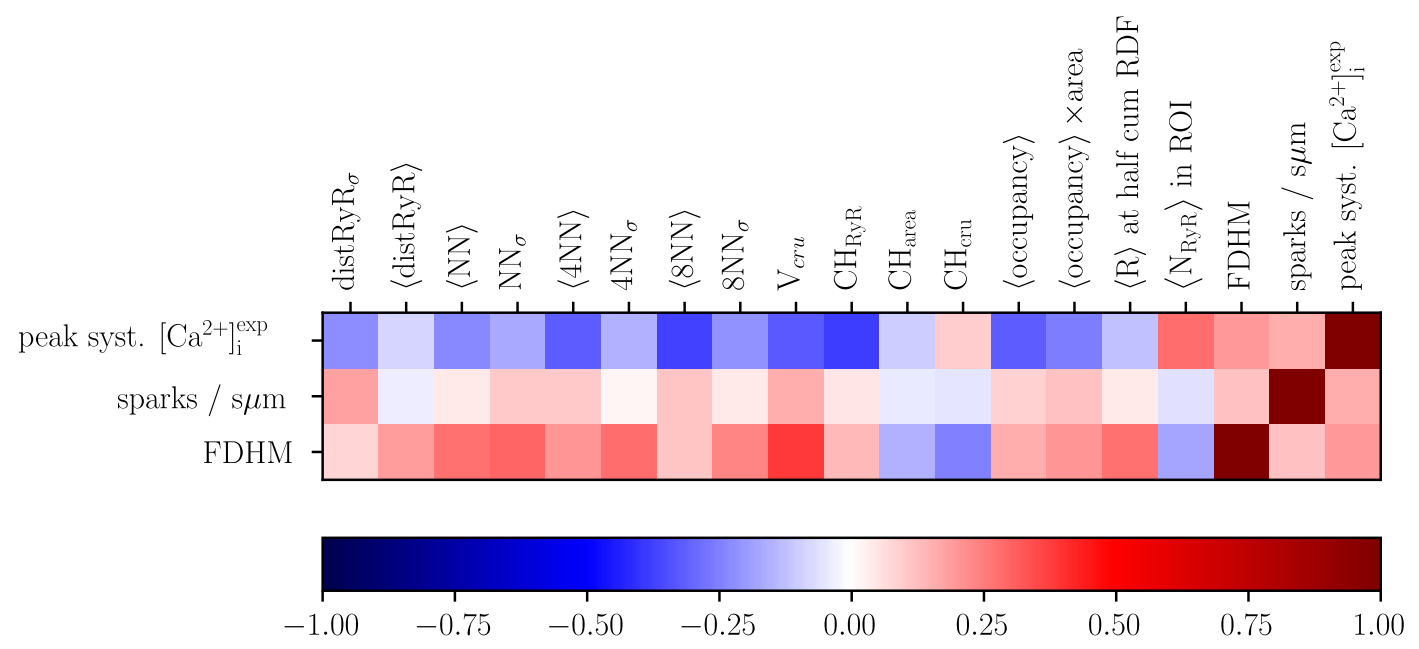

Figure 3.10 - Cross-correlation of three spark biomarkers peak calcium concentration, sparks and FDHM on the y-axis with measures for RyR cluster shape and structure on the X-axis, where the biomarkers are listed again as well. One corresponds to full correlation, while -1 shows total anti-correlated measures.

of 1 represents full correlation and -1 total anti-correlation. Nearest neighbours as well as CRU volume are negatively correlated with $\mathrm{APD}_{90}$ and calcium concentrations and not well correlated with the time to peak, thus cannot be considered as a suitable measure for describing the cluster characteristics with respect of the selected biomarkers. The input parameters $\langle$ distRyR $\rangle$ and distRyR $\mathrm{R}_{\sigma}$ are less well correlated to the chosen biomarkers and, as already mentioned, lack information on the actual cluster shape and size and are discarded as well. The mean radius at half cumulative radial distribution function would deliver more information in this regard, but is strongly uncorrelated to time to peak and $\mathrm{APD}_{90}$. A measure with good positive correlations on $\mathrm{APD}_{90}$ and calcium concentrations is the mean number of RyRs in the region of interest, but it is not well correlated with the time to peak and again it reveals no additional information on the cluster shape. The convex hull $\mathrm{CH}$, instead, has this information and is furthermore well positively correlated with all AP biomarkers.

In figure 3.10 the correlation between the geometry measures and the main spark biomarkers is shown. While the peak calcium value is positively correlated only with the convex hull and the mean number of RyRs in the region of interest, the sparks and the FDHM are positively correlated with nearly all considered measures. They only have a slightly negative correlation with the convex hull $\mathrm{CH}$ and mean number of RyRs $\left\langle\mathrm{N}_{\mathrm{RyR}}\right\rangle$ in ROI. Nevertheless the former correlation is balanced by the positive impact the mean occupancy has on the two biomarkers. Because of this behaviour and since, again, the convex hull $\mathrm{CH}$ and the occupancy are well related (see section 3.4.3), we can choose the occupancy to be the descriptive measure for the cleft geometry. 


\subsubsection{Convex Hull and Occupancy}

The convex hull can be pictured geometrically as the minimal polygon enclosing a group of points and is shown for an example cleft in figure 3.11a as the blue line. The convex hull perfectly grasps the shape of a channel cluster, but lacks information on the packing. In order to account for the channel density we computed the occupancy based on the considerations done by Bertamini and coauthors in [112]. The occupancy, partly visualised for the same example cleft in figure $3.11 \mathrm{~b}$, is defined via:

$$
1-\frac{\Omega}{\mathcal{A}}
$$

where $\mathcal{A}$ is the sum of all areas of influence (green circles in figure 3.11b) around each RyR and $\Omega$ is the sum of all overlap area. The circular areas of influence are determined as the area enclosed by the convex hull divided by the number of channels. The occupancy is thus directly related to the convex hull. From equation (3.11) it is apparent that the smaller the occupancy is the closer the RyRs are packed, while a value of 1 represents the case, in which channels are far apart from each other and share no area of interest.

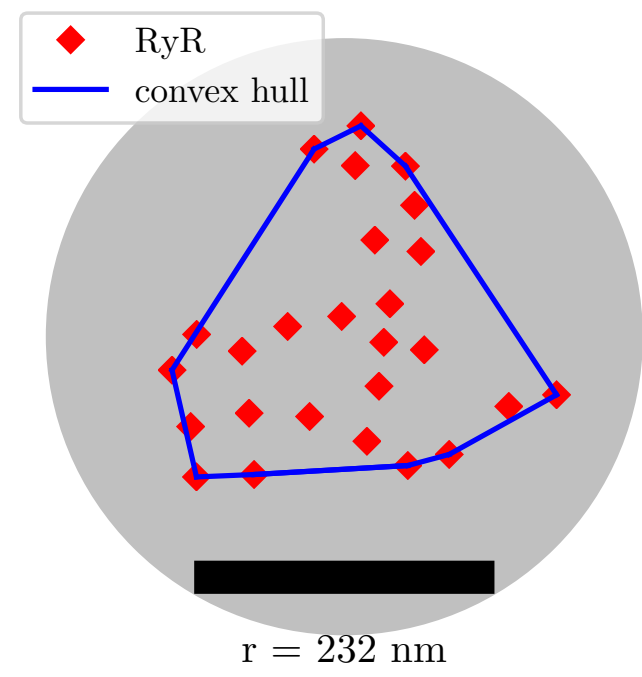

(a) - RyR positions (red diamonds) as they are enclosed by a convex hull (blue line). The area inside the convex hull is divided by the total number of RyRs to gain the area of interest of each channel.

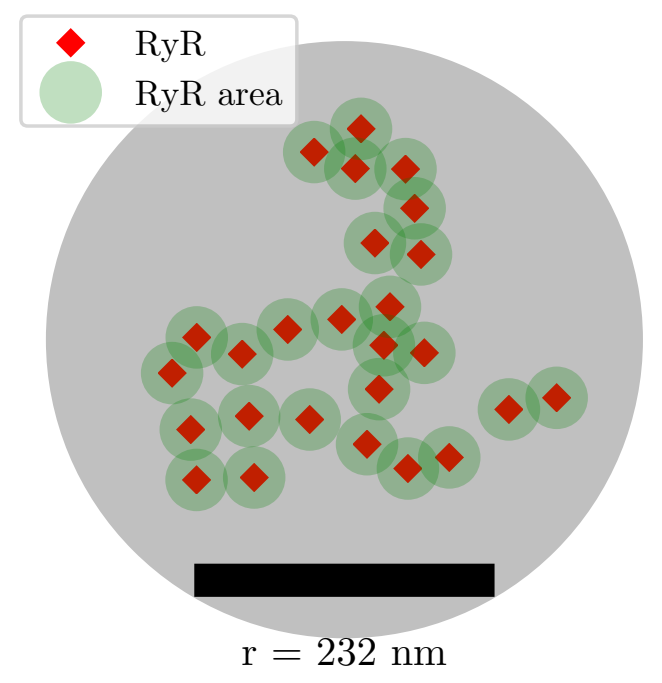

(b) - Area of interest (green overlapping circles) of each RyR (red diamonds). Note: the diameter of the circles does not match with the actual computed area of interest; the depicted circles are descriptive sketches.

Figure 3.11 - Representations of convex hull as a blue line and areas of interest for the computation of the occupancy as green circles of one example cleft showing the positions of RyRs computed with the Soeller placement as red diamonds. 
This feature of the occupancy makes it a valid candidate for a measure of the RyR cluster density. A comparison of the biomarker behaviour for varying convex hull and occupancy showed only few differences in the trends. This fact together with the similarity to the occupancy and the convex hull ultimately persuaded us to choose the occupancy as a measure for RyR cluster characteristics.

The occupancy in the simulations comparing regular and Soeller placement already reveals the differences between the two. The former has a value of the mean occupancy $(\approx 0.36)$ smaller than the latter $(\approx 0.49)$ already pointing at a higher packing density of RyRs when on a regular lattice.

With the occupancy being assessed as the measure describing the clusters we then considered the population of simulation runs, in which $\langle$ distRyR $\rangle$ and distRyR ${ }_{\sigma}$ were varied. We considered the biomarkers selected for the comparison of regular and Soeller placement (see section 3.4.1) and their behaviour upon changes of the mean occupancy. In figures 10 and 11 in section 4.1 and in [50] the biomarkers of AP and spark simulations are depicted as functions of the computed mean occupancy. The two figures are described and discussed in detail in the section on "Functional consequences of geometrical properties of RyR clusters" in section 4.1 and in [50].

A central point in the comparison of the placement protocols is not only the apparent differences between two methods, but also those that arise from variations within one method, in our case the Soeller placement. These differences highlight the strong impact of changes in the micro-domain cellular geometry and structure on the ECC on a mesoscopic level underlining the importance of inclusion of detailed subcellular structures in mathematical models. 



\section{Chapter 4}

\section{Impact of Structure Modifications on Cardiomyocyte Functionality}

\subsection{Dyadic Structure-Function Relation in Cardiomyocytes}

This section consists of the article [50] published as:

F. G. Cosi, W. Giese, W. Neubert, S. Luther, N. Chamakuri, U. Parlitz, and M. Falcke. "Multiscale Modeling of Dyadic Structure-Function Relation in Ventricular Cardiac Myocytes". en. In: Biophysical Journal (Sept. 2019). DOI: $10.1016 /$ j.bpj.2019.09.023

under the Creative Commons License (CC BY-NC-ND 4.0) ${ }^{1}$ with myself and Wolfgang Giese as equally contributing first authors.

Wolfgang Giese and I implemented the Soeller placement into cleftdyn, performed the simulations, created an analysing framework for the determination and analysis of the biomarkers and carried out all shown analysis. Wilhelm Neubert and Nagaiah Chamakuri were responsible for the maintenance and optimisation of the cleftdyn code. Martin Falcke, Ulrich Parlitz, Wolfgang Giese and I did design the main research ideas on influences of channel placements on the model's biomarkers, on the choice of the measures for the description of the cleft geometry and on the use of uncertainty quantification and polynomial chaos expansion regression as analysing tools. The graphs in figures 1, 3, 8, 9 and 10 were done by Wolfgang Giese, I created figures 2, 5 and 7, of which the last two I designed together with Wolfgang Giese as it is the case for figures 4 and 6 , which were in the end created by him. All authors contributed to the writing of the manuscript.

\footnotetext{
${ }^{1}$ https://creativecommons.org/licenses/by-nc-nd/4.0/
} 


\title{
Multiscale Modeling of Dyadic Structure-Function Relation in Ventricular Cardiac Myocytes
}

\author{
Filippo G. Cosi, ${ }^{1,4,5}$ Wolfgang Giese, ${ }^{2}$ Wilhelm Neubert, ${ }^{2}$ Stefan Luther, ${ }^{1,4,5}$ Nagaiah Chamakuri, ${ }^{3}$ \\ Ulrich Parlitz, ${ }^{1,4,5}$ and Martin Falcke ${ }^{2,6,7, *}$ \\ ${ }^{1}$ Max Planck Institute for Dynamics and Self-Organization, Göttingen, Germany; ${ }^{2}$ Max Delbrück Center for Molecular Medicine in the Helmholtz \\ Association, Berlin, Germany; ${ }^{3}$ Institute of Applied Mathematics, University of Hohenheim, Stuttgart, Germany; ${ }^{4}$ Georg-August-Universität \\ Göttingen, Institute for the Dynamics of Complex Systems, Göttingen, Germany; ${ }^{5}$ DZHK (German Center for Cardiovascular Research), \\ Partner Site Göttingen, Göttingen, Germany; ${ }^{6}$ DZHK (German Center for Cardiovascular Research), Partner Site Berlin, Berlin, Germany; and \\ ${ }^{7}$ Department of Physics, Humboldt University Berlin, Germany
}

ABSTRACT Cardiovascular disease is often related to defects of subcellular components in cardiac myocytes, specifically in the dyadic cleft, which include changes in cleft geometry and channel placement. Modeling of these pathological changes requires both spatially resolved cleft as well as whole cell level descriptions. We use a multiscale model to create dyadic structure-function relationships to explore the impact of molecular changes on whole cell electrophysiology and calcium cycling. This multiscale model incorporates stochastic simulation of individual L-type calcium channels and ryanodine receptor channels, spatially detailed concentration dynamics in dyadic clefts, rabbit membrane potential dynamics, and a system of partial differential equations for myoplasmic and lumenal free $\mathrm{Ca}^{2+}$ and $\mathrm{Ca}^{2+}$-binding molecules in the bulk of the cell. We found action potential duration, systolic, and diastolic $\left[\mathrm{Ca}^{2+}\right]$ to respond most sensitively to changes in L-type calcium channel current. The ryanodine receptor channel cluster structure inside dyadic clefts was found to affect all biomarkers investigated. The shape of clusters observed in experiments by Jayasinghe et al. and channel density within the cluster (characterized by mean occupancy) showed the strongest correlation to the effects on biomarkers.

SIGNIFICANCE Diseases such as myocardial infarction, aortic stenosis, tachycardia, hypertension, chronic ischemia, and atrial fibrillation have been related to changes inside the dyadic cleft, which is a subvolume of cardiac myocytes of $\sim 10^{-17} \mathrm{I}$ (typical cell volume $10^{-11} \mathrm{I}$ ). However, exploration of the relation between subdyadic structures and disease is difficult because such microscopic structures in cells are in many cases not amenable to experimental manipulation, or experiments addressing them might not allow for simultaneous observation of cellular responses. Multiscale mathematical models can explore the relation between microscopic structures and cellular response. We show by mathematical modeling that the geometric properties of ryanodine receptor channel clusters within dyadic clefts affect cellular responses.

\section{INTRODUCTION}

The functioning of the heart is based on the precisely controlled contraction of its cardiac myocytes coordinated across the muscle by waves of membrane potential depolarizations (action potentials (APs)) emanating from the sinoatrial node. On the level of an individual cardiac myocyte, L-type $\mathrm{Ca}^{2+}$ channels (LCCs) open during an AP and trigger the release of $\mathrm{Ca}^{2+}$ from the sarcoplasmic reticulum (SR), which is the main intracellular $\mathrm{Ca}^{2+}$ storage compartment.

Submitted May 31, 2019, and accepted for publication September 16, 2019.

*Correspondence: martin.falcke@mdc-berlin.de

Filippo G. Cosi and Wolfgang Giese contributed equally to this work.

Editor: Daniel Beard

https://doi.org/10.1016/j.bpj.2019.09.023

(C) 2019 Biophysical Society.

This is an open access article under the CC BY-NC-ND license (http:// creativecommons.org/licenses/by-nc-nd/4.0/)
The entailing global $\mathrm{Ca}^{2+}$ increase causes the binding of molecular motors to actin filaments in the sarcomeres and initiates contraction.

The SR forms a network of tubes that extend throughout the interior of the cell and can be divided into two main components known as junctional SR (jSR) and network $\mathrm{SR}$ (nSR). The cardiac myocyte is penetrated by a network of transverse tubules, which are plasma membrane invaginations that approach the jSR and thereby form small cellular subvolumes (see Fig. 1). These subvolumes, which have a height of 10-15 nm, are called dyadic clefts. Action potential gated LCC opening leads to calcium-induced calcium release (CICR) through ryanodine receptor channels (RyRs) in the jSR membrane. $\mathrm{Ca}^{2+}$ induces its own release because the opening probability of the RyRs increases with 
Cosi et al.

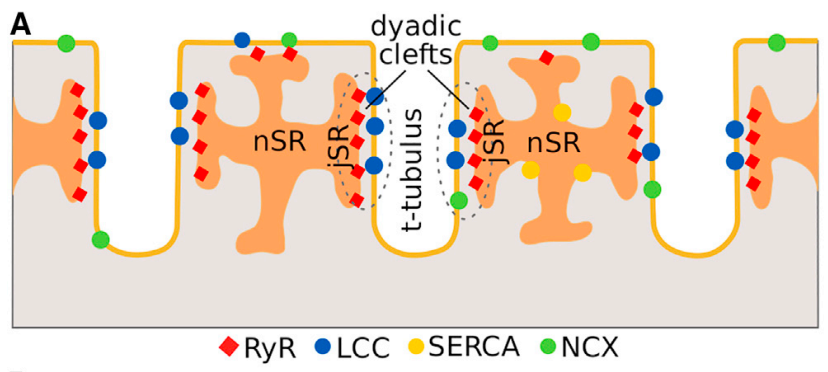

B

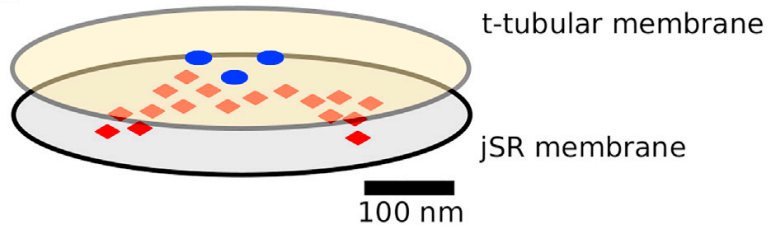

FIGURE 1 (A) Sketch of cellular level organization and transverse tubular structure in the cardiac myocyte. $(B)$ Molecular level arrangement of LCCs and RyRs in a single dyadic cleft is shown. To see this figure in color, go online.

the local $\mathrm{Ca}^{2+}$ concentration at the channel. CICR is facilitated by the co-localization of LCCs in the transverse tubules membrane on the one side of a dyadic cleft and RyRs in the jSR membrane on the other side. The RyRs together with the LCCs and the associated jSR structure comprise the calcium release unit (CRU). Release from CRUs provides the $\mathrm{Ca}^{2+}$ triggering contraction of the sarcomeres. CRUs are concentrated in the structures connecting sarcomeres-the $\mathrm{z}$-disks. The CRUs are arranged within $\mathrm{z}$-disks with distances of less than $1 \mu \mathrm{m}$. The $\mathrm{z}$-disks form a regular stack with a spacing of $\sim 2 \mu \mathrm{m}$.

CRUs behave stochastically because they contain a small number of ion channels. Because of CICR, they are excitable and can form sparks, which are the elementary events of $\mathrm{Ca}^{2+}$ release. Cooperation of several CRUs via CICR may generate unwanted $\mathrm{Ca}^{2+}$ waves. These processes occur on different time and length scales (see Fig. S1). The $\left[\mathrm{Ca}^{2+}\right]$ changes inside the dyadic cleft happen within a few milliseconds; SR dynamics, on the other hand, act on a timescale of up to tens of seconds. We have spatial scales ranging from tens of nanometers in the dyadic cleft up to $100 \mu \mathrm{m}$ in cell size. To account for these temporal and spatial scales, multiscale models with spatially distributed $\mathrm{Ca}^{2+}$ release sites have been developed (1-6). The model used here simulates the behavior of individual RyR and L-type calcium channels as well as the concentration gradients inside clefts. On cell level, we simulate the membrane potential and concentration dynamics. We do not use the approximation by spatial compartments for the bulk concentration dynamics but simulate the corresponding partial differential equations with the numerically required spatial resolution $(1,6)$.

Common challenges with detailed multiscale modeling are the parameterization of the model by reproducing the values of a set of measured biomarkers (biomarkers are measurable properties of cells characterizing cell behavior) and the quantification of unknown values of parameters (710) because of the large number of simulations required for these purposes. Methods to quantify the relationship of variability and uncertainty of model inputs (parameter values) to outputs (simulated biomarker values), which are based on the construction of a response surface, have been recently suggested $(7,8,11,12)$ and may spare many simulation runs. Exploiting the multiscale abilities of the model, we use biomarkers on different time and length scales for APs (seven biomarkers) and $\mathrm{Ca}^{2+}$ sparks (four biomarkers) to adapt our model to rabbit experimental results. Our systematic quantification of the relation between parameter and biomarker values uses an approximation by sums of polynomials (polynomial chaos expansion $(12,13,14)$ ). Sensitivity analysis identifies the parameters dominating the control of biomarker values - both in the mathematical sense of it and as the model's suggestion for most efficient control of the cell state (e.g., by post-translational modifications).

Diseases such as myocardial infarction, aortic stenosis, tachycardia, hypertension, and chronic ischemia are frequently related to changes in the dyadic cleft $(15,16)$, which motivated several studies in recent years focusing on the details of the placement of RyR channels inside it. It turned out not to be on a square lattice as assumed before but to be less regular with respect to size and geometrical properties (16-24). The geometrical analysis revealed that channel positions in a cluster have random components and that cluster area is elongated in one direction rather than quadratic or circular (17-20,24,25). Although the cluster size heterogeneity has been related to spark probability (23), the functional consequences of the geometrical properties of RyR clusters are not so obvious yet. Modeling can investigate them only if intradyadic gradients are taken into account as our approach does and other studies on the CRU level did $(22,25,26)$. We address the functional consequences of channel placement on CRU and cell level and compare them between the regular arrangement and configurations with increasing irregularity. To that end, we choose the rules of channel placement provided by Jayasinghe et al. (17) to generate cluster geometries and channel locations similar to experimental observation. We put CRUs with these cluster geometries into a ventricular cell model to study the relation between cluster structure and cell function.

\section{MATERIALS AND METHODS}

\section{Mathematical model and methods}

The mathematical model comprises whole cell dynamics as well as local molecular events (see Figs. 1 and S1). On the finest level, individual channels are represented as continuous time Markov chains, coupled by local gradients inside the dyadic space. Cell wide diffusion of $\left[\mathrm{Ca}^{2+}\right]$ and its buffers is modeled by partial differential equations, which also include the fluxes generated by SR/endoplasmic reticulum $\mathrm{Ca}^{2+}$-ATPase (SERCA), NCX $\left(\mathrm{Na}^{+} / \mathrm{Ca}^{2+}\right.$ exchanger), and the CRUs. Spatially averaged variables 
comprise membrane potential, $\left[\mathrm{Na}^{+}\right]$, and $\left[\mathrm{K}^{+}\right]$which are generally assumed to not exhibit strong gradients on the subcellular level. A detailed description of the model can be found in $(1,6)$ and Supporting Materials and Methods.

The finite element simulation toolbox DUNE has been used to solve the model equations $(27,28)$. A complete description of the numerical approach is given in $(1,6)$ and a short overview in Supplementary Materials and Methods.

\section{Channel placement model}

Mathematical models describing cellular calcium dynamics in cardiac myocytes generally omit a spatially resolved description of the single CRUs depicting them as point sources and/or neglect the internal structure of channel arrays inside dyadic clefts by assuming spatially homogeneous $\mathrm{Ca}^{2+}$ concentration (4,29-31). However, we take them into account as required by our investigation on RyR placement $(1,6,32,33)$. To determine the RyR arrangement in the cleft, we use the placement algorithm suggested by Jayasinghe et al. (17), which provides channel locations closely resembling their experimental data. This placement algorithm determines channel locations as the sequence of positions in a two dimensional random walk with as many steps as channels in the dyad. The first RyR is placed in the center of the dyadic cleft. The position of the second RyR is found by a step in a random direction and with random length. The step length is drawn from a normal distribution (mean $\mu_{\mathrm{RyR}}=40.1 \mathrm{~nm}$, SD $\sigma_{\mathrm{RyR}}=7.4 \mathrm{~nm}$ from (17)) with a cutoff accounting for the channel molecule diameter of $30 \mathrm{~nm}$ (34). The angle defining the direction is drawn from a uniform distribution in $[0,2 \pi]$. Subsequent steps to channel positions obey the same rules plus the additional requirement to steer clear of existing channel molecules (excluded volume).

The first LCC channel is positioned at the center of the RyR cluster. In the following, LCCs are placed on a regular grid as in (1), again with a minimal distance of $30 \mathrm{~nm}$ from any other channel. Two examples of channel locations in a dyadic cleft generated this way are shown in Fig. $2 C$.

\section{Sensitivity analysis and construction of a response surface}

We generated a population of models by varying five crucial model parameters using Latin hypercube sampling (35). The hypercube was formed by the axes in the parameter space representing $k_{\text {plus }}, k_{\text {close }}, g_{\text {RyR }}, g_{\mathrm{LCC}}$, and $V_{\mathrm{P} \text {,max }}$. All five parameters were varied by a factor of 10 (see Table 1). The choice of the parameter ranges was based on values in the literature $(1,36,37)$. The literature values may depart slightly from the chosen ranges because of different pacing cycle lengths. The pacing cycle length used in our simulations is $350 \mathrm{~ms}$. The model simulations have identical initial conditions except for the stochasticity of the geometric channel arrangement and selected model parameters. All samples were run for the same simulation time. The resulting set of simulation results was analyzed by Bayesian linear regression (38) and polynomial chaos expansion (13) to obtain local and global parameter dependencies.

Bayesian linear regression was used to obtain an estimate of local parameter sensitivity coefficients. The model sensitivity $s_{X Y}$ from the linear fit was computed from the following:

$$
s_{X Y}=\frac{X_{\text {ref }}}{Y_{\text {ref }}} S_{X Y}
$$

where $S_{X Y}$ is the slope (along parameter $\mathrm{X}$ ) from multiple linear regression in a specified neighborhood of a reference parameter set (marked in Figs. 5 and 7 as a red cross). Here $X_{\text {ref }}$ denotes the corresponding parameter value, and $Y_{\text {ref }}$ is the corresponding reference value of the biomarker. We can read off the local strength, direction, and uncertainty of output change with respect to the variation of a selected parameter from these sensitivity coef-
A

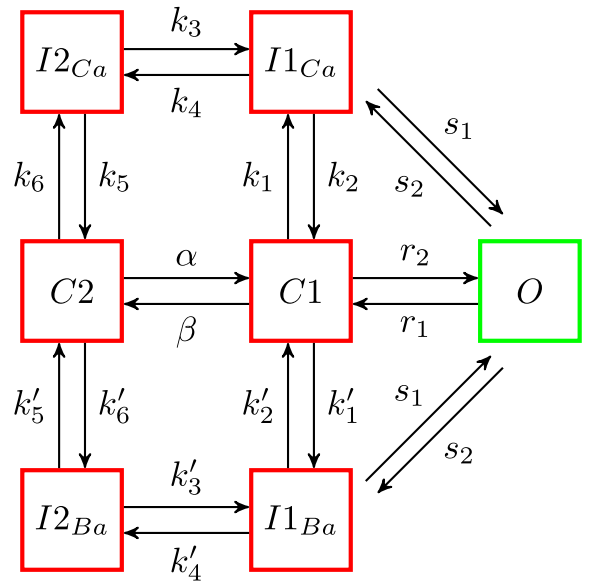

B

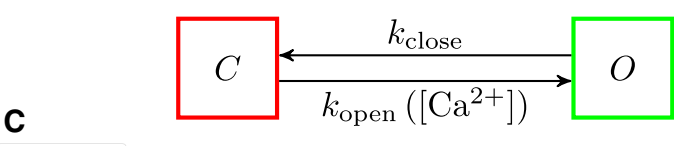

RyR
- LCC
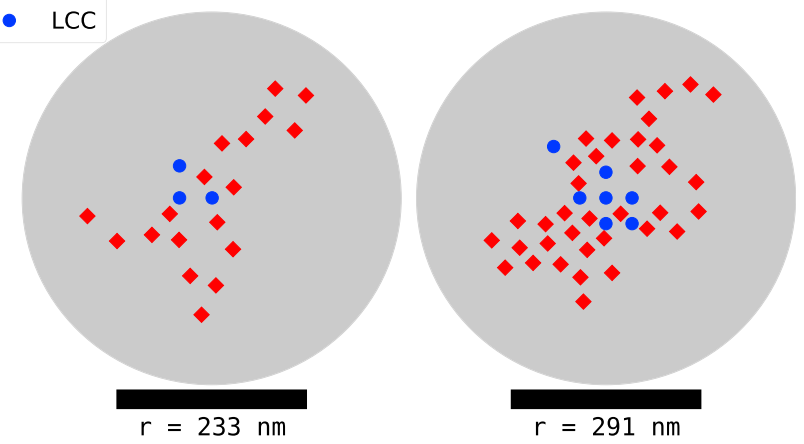

FIGURE 2 (A) L-type $\mathrm{Ca}^{2+}$ channel (LCC) state scheme with the open state marked in green (36). (B) Ryanodine receptor (RyR) state scheme with the open state marked in green (26); the open rate $k_{\text {open }}$ depends on dyadic as well as on jSR $\left[\mathrm{Ca}^{2+}\right]$ as described in more detail in Supporting Materials and Methods. (C) Shown are two examples of placements of LCCs (blue circles) and RyRs (red diamonds) in single dyadic clefts with radii $r$. To see this figure in color, go online.

ficients. Because the mathematical model is stochastic, the output cannot be predicted with absolute certainty but with some probability only. We therefore used Bayesian linear regression to obtain a proper quantification of the uncertainty in the predictions. The algorithms were implemented using the Python library Edward (39) and TensorFlow (40) toolboxes. Although these sensitivities are based on a linear regression, we complemented our investigations by calculating Sobol coefficients (see Fig. S3), which serve as a measure of global sensitivities for nonlinear models (41).

To quantify the effect parameters have on the biomarkers, we used an approximation method known as polynomial chaos expansion. For the polynomial chaos expansion, an orthonormal basis of polynomials was generated by using a Python-specific library called Chaospy (42). We assumed uniform distribution of the input parameters and therefore used Legendre polynomials for the regression fit. Using a polynomial degree $p$ and a number of parameters $d$, the number of polynomial coefficients, which have to be determined, can be calculated from:

$$
n=\left(\begin{array}{c}
d+p \\
d
\end{array}\right)
$$


Cosi et al.

TABLE 1 Parameters, Sampling Ranges, and Suggested Parameter Set for Spark and Action Potential Simulations

\begin{tabular}{lcccc}
\hline Parameter & Description & Sampling Range & Suggested Value & Accepted Range \\
\hline$k_{\text {plus }}\left[\mathrm{ms}^{-1} \mu \mathrm{M}^{-\eta}\right]$ & RyR opening rate & $5.0 \times 10^{-5}-5.0 \times 10^{-4}$ & $1.5 \times 10^{-4}$ & $(1.1-2.3) \times 10^{-4}$ \\
$k_{\text {close }}\left[\mathrm{ms}^{-1}\right]$ & RyR closing rate & $0.1-1.0$ & 0.5 & $0.28-0.55$ \\
$g_{\text {RyR }}\left[\mu \mathrm{m}^{3} \mathrm{~s}^{-1}\right]$ & RyR Ca & $3.0 \times 10^{-4}-3.0 \times 10^{-3}$ & $7.5 \times 10^{-4}$ & $(5.9-8.4) \times 10^{-4}$ \\
$g_{\mathrm{LCC}}\left[\mu \mathrm{m}^{3} \mathrm{~s}^{-1}\right]$ & LCC Ca ${ }^{2+}$ permeability & $4.5 \times 10^{-4}-4.5 \times 10^{-3}$ & $3.2 \times 10^{-3}$ & $(1.7-3.4) \times 10^{-3}$ \\
$V_{\mathrm{P}, \max }\left[\mu \mathrm{M} \mathrm{ms}^{-1}\right]$ & maximal SERCA uptake rate & $0.15-1.5$ & 0.55 & $0.08-0.71$ \\
\hline
\end{tabular}

The output stays in the literature range of all biomarker values, if the corresponding parameter is varied within the range given in the fifth column (accepted range), whereas other parameter values are kept at the value in the fourth column (suggested value).

The required number of data points usually exceeds the number of coefficients by at least a factor of 2-3 to prevent overfitting (43). To obtain an optimal regression and polynomial degree, we quantified the commonly used least-squares fit error and the cross-validation error as explained in Supporting Materials and Methods.

\section{RESULTS}

The simulated time course of the $\mathrm{Ca}^{2+}$ concentration inside a dyadic cleft during an AP is illustrated in Fig. 3 and Video S1. Gradients comprise three orders of magnitude (0.1$150 \mu \mathrm{M})$ upon the opening of the first channel. The concentration outside the cleft space changes quickly, such that we observe gradients from $\sim 150 \mu \mathrm{M}$ at the boundary of the cleft to $\sim 300 \mu \mathrm{M}$ at open channels later during the event. Hence, the opening rate of RyRs close to open channels is initially six orders of magnitude and, later into the spark, is $\sim 4$ times faster than the rate of channels further away (see Eq. S11; Table S2). The simulations illustrate the strong impact of gradients on the transition from one open LCC (or RyR, quark) to a spark and the quantitative effect on the calcium transient. This applies throughout an AP, as Video S1 shows.

The jSR concentration decreases rapidly upon the onset of release (Fig. 3). In case of sparks, this helps terminating release due to decreasing release current and consequently less coupling of RyRs by CICR. This mechanism is in agreement with earlier studies $(25,26,44)$. In case of AP simulations, the jSR concentration continues to decrease on average till about the end of the membrane potential plateau (Fig. 3).
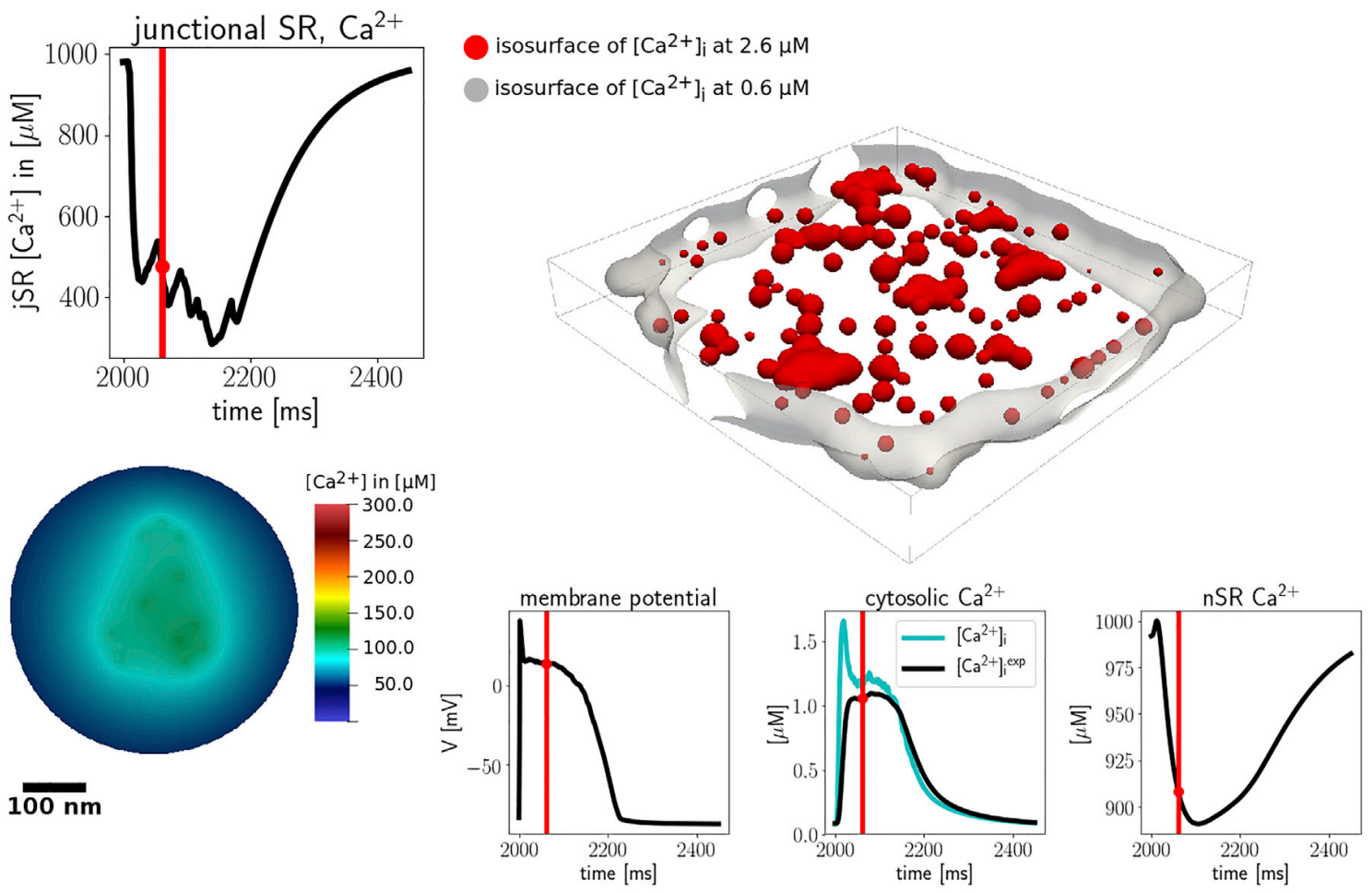

FIGURE 3 Multiscale simulation. Shown is a snapshot of the concentration profile in a dyadic cleft and the jSR concentration time course of this CRU. A snapshot of isoconcentration surfaces $(0.6 \mu \mathrm{M}, 2.6 \mu \mathrm{M})$ of the cytosolic concentration $\left[\mathrm{Ca}^{2+}\right]_{\mathrm{i}}$ of the whole $\mathrm{z}$-disk $(15 \mu \mathrm{m} \times 15 \mu \mathrm{m} \times 2 \mu \mathrm{m})$ with 320 dyadic clefts and the time course of the membrane potential, average $\left[\mathrm{Ca}^{2+}\right]_{\mathrm{i}}$, and average nSR $\left[\mathrm{Ca}^{2+}\right]$ are shown on the right-hand side. The red line indicates the time point at which the snapshots were captured. A corresponding simulation for one AP is shown in Video S1. To see this figure in color, go online. 
Fig. 3 also shows the cytosolic concentration $\left[\mathrm{Ca}^{2+}\right]_{\mathrm{i}}$ caused by the release from all CRUs in the z-disk $(15 \mu \mathrm{m}$ $\times 15 \mu \mathrm{m} \times 2 \mu \mathrm{m})$ and the membrane potential, average $\left[\mathrm{Ca}^{2+}\right]_{\mathrm{i}}$, and average nSR concentration. The variable size of the volumes enclosed by the $2.6 \mu \mathrm{M}$ isoconcentration surface illustrates the randomness and heterogeneity of release events. Refined numerical grids around CRUs guarantee the faithful simulation of $\mathrm{Ca}^{2+}$ and buffer diffusion between them (6). In that way, we can simulate the concentration dynamics from subdyadic to cellular length and timescales.

To facilitate the comparison of experimentally measured and simulated $\left[\mathrm{Ca}^{2+}\right]$, we simulated a fluorescent buffer (see Fig. S4). This allowed us to emulate the approximation of $\left[\mathrm{Ca}^{2+}\right]_{\mathrm{i}}$ as it would be measured by a single wavelength Fluo-4 experimental recording using an in vitro calibration approach as described in (45):

$$
\left[\mathrm{Ca}^{2+}\right]_{i}^{\exp }=K_{d} \frac{F-F_{\min }}{F_{\max }-F}
$$

where $K_{d}$ is the dissociation constant of Fluo- $4, F$ is the experimentally measured fluorescence intensity (the spatial average of $\left.b_{f}\right), F_{\max }$ is the measured fluorescence intensity in $\mathrm{Ca}^{2+}$-saturated dye (here, this is set as $b_{f}^{\text {tot }}$ ), and $F_{\text {min }}$ is the measured fluorescence intensity in the absence of $\mathrm{Ca}^{2+}$ (here, set to zero).

\section{Quantification of parameter values based on biomarkers}

A population of simulated cells was generated as described in Materials and Methods. We identified valid parameter sets by filtering all simulation results for those providing biomarker values in the ranges stated in literature (see Tables 2 and 3). The biomarker resting membrane potential, maximum membrane potential, dome membrane potential, and $\left[\mathrm{Na}^{+}\right]_{i}$ (see Table 2) are mainly determined and met by the Mahajan electrophysiology model we use and have essentially not been affected by the parameter variations considered here (36). Results of AP simulations were filtered by taking the biomarkers APD90, peak systolic $\left[\mathrm{Ca}^{2+}\right]$, and mean diastolic $\left[\mathrm{Ca}^{2+}\right]$ into account. The spark biomarkers used in the filtering of the spark simulations are the spark rate (i.e., events with at least two simulta-

TABLE 2 Biomarkers Ranges for Action Potentials

\begin{tabular}{lcc}
\hline Biomarker & Range & Description \\
\hline Max $V_{m}$ & $46 \pm 4.5 \mathrm{mV}$ & maximal value of AP peaks \\
Resting $V_{m}$ & $-77.4 \pm 3.9 \mathrm{mV}$ & resting value of the AP \\
Dome $V_{m}$ & $15.2 \pm 10.1 \mathrm{mV}$ & peak in the plateau phase \\
APD90 & $150-200 \mathrm{~ms}$ & APD at $90 \%$ \\
Systolic $\left[\mathrm{Ca}^{2+}\right]$ & $0.6-1.2 \mu \mathrm{M}$ & peak systolic calcium \\
Diastolic $\left[\mathrm{Ca}^{2+}\right]$ & $0.1-0.25 \mu \mathrm{M}$ & diastolic calcium \\
{$\left[\mathrm{Na}^{+}\right]_{i}$} & $10.5-11.5 \mathrm{mM}$ & intracellular sodium \\
\hline
\end{tabular}

Table $\mathrm{S} 1$ also lists references.
TABLE 3 Biomarker Ranges for $\mathrm{Ca}^{2+}$ Sparks

\begin{tabular}{lcc}
\hline Biomarker & Range & Description \\
\hline FDHM & $8.0-17.5 \mathrm{~ms}$ & FDHM \\
Spark rate & $1-5 \mu \mathrm{m}^{-1} \mathrm{~s}^{-1}$ & $\begin{array}{c}\text { number of sparks per } \mu \mathrm{m} \text { cell } \\
\text { and second }\end{array}$ \\
$\begin{array}{l}\text { Quark to spark } \\
\text { ratio }\end{array}$ & $0.2-1.1$ & $\begin{array}{c}\text { number of quarks/number of sparks } \\
\text { Peak }\left[\mathrm{Ca}^{2+}\right]\end{array}$ \\
Peak $\left[\mathrm{Ca}^{2+}\right]_{i}^{\exp }$ & $0.0-22.0 \mu \mathrm{M}$ & average maximal $\left[\mathrm{Ca}^{2+}\right]$ \\
\hline
\end{tabular}

Table $\mathrm{S} 1$ also lists references.

neously open RyRs in the same cleft), the average FDHM, the mean of the $\mathrm{Ca}^{2+}$ peak value of sparks, and the ratio between quarks and sparks. Quarks are events in which exactly one RyR opens in a given cleft. The overlap of both filtering results led to the suggested parameter value set in Table 1. We performed 281 simulations for APs. Of those, 23 parameter sets passed the biomarker ranges stated above. Out of the $297 \mathrm{Ca}^{2+}$ spark simulations, 20 simulations passed the ranges for the $\mathrm{Ca}^{2+}$ sparks. The suggested value (Table 1, fourth column) fulfills the requirement for all seven biomarkers.

\section{Sensitivity analysis and response surfaces of AP biomarkers}

Sensitivity analysis provides information on how changes of input parameters affect a particular biomarker value. We have chosen to vary the five parameters $k_{\text {plus }}, k_{\text {close }}, g_{\text {RyR }}$, $g_{\mathrm{LCC}}$, and $V_{\mathrm{P}, \max }$ in this analysis. All of them are related to $\mathrm{Ca}^{2+}$ as the focus of this study. The parameters setting the RyR open probability $\left(k_{\text {plus }}, k_{\text {close }}\right)$ and SERCA uptake $\left(V_{\mathrm{P}, \mathrm{max}}\right)$ were chosen because they are targets of drugs or post-translational modifications. We vary the RyR conductivity $g_{\text {RyR }}$ because the in vivo single channel current is not well known. The LCC conductivity $g_{\text {LCC }}$ turned out to be an important parameter in preliminary simulations.

We have chosen the suggested values of the parameters in Table 1 as reference for all sensitivities in this study. Fig. 4 shows the sensitivities for the AP biomarkers (APD90, peak systolic $\left[\mathrm{Ca}^{2+}\right]$, and mean diastolic $\left[\mathrm{Ca}^{2+}\right]$ ) versus the varied parameters. The LCC permeability $g_{\mathrm{LCC}}$ has the strongest positive impact on all three biomarkers (Fig. 4). The positive correlation of APD90 with $g_{\text {LCC }}$ we observe is in line with results by Britton et al. (46). AP durations (APDs) are positively influenced by the RyR opening rate $k_{\text {plus }}$ and negatively by their closing rate $k_{\text {close }}$, whereas for the systolic $\left[\mathrm{Ca}^{2+}\right]_{\mathrm{i}}$ peak and diastolic $\left[\mathrm{Ca}^{2+}\right]_{\mathrm{i}}$, the opposite is true. The influence of the opening and closing rates of the RyRs on the APD mediates the effect these two parameters have on the $\left[\mathrm{Ca}^{2+}\right]_{i}$ values. An increase of $k_{\text {plus }}$ decreases $\left[\mathrm{Ca}^{2+}\right]_{\mathrm{i}}$ because it prolongs the AP. This entails longer $\mathrm{Ca}^{2+}$ release, which, in the end, reduces SR $\left[\mathrm{Ca}^{2+}\right]$ and the release and leak currents. We see the opposite effect when increasing $k_{\text {close }}$. It 
Cosi et al.

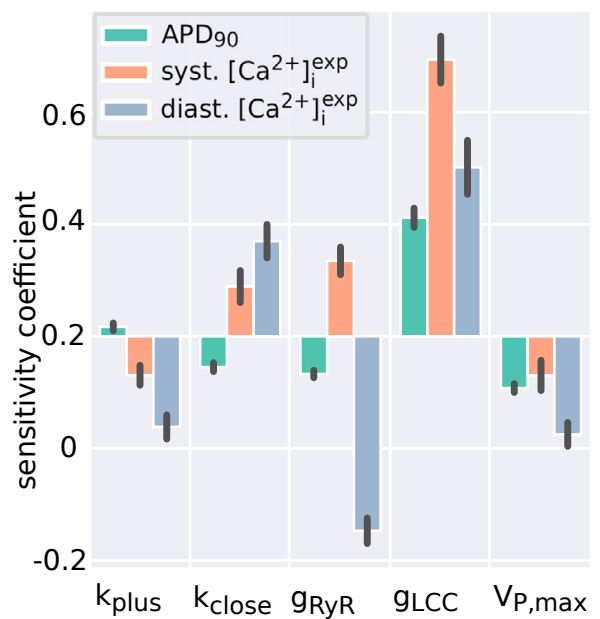

FIGURE 4 Sensitivity values for APD90, systolic $\left[\mathrm{Ca}^{2+}\right]_{i}^{\text {exp }}$, and diastolic $\left[\mathrm{Ca}^{2+}\right]_{i}^{\text {exp }}$. Black bars represent the SD for the sensitivity coefficients obtained from Bayesian inference and indicate the uncertainty in the estimates. To see this figure in color, go online.

shortens the AP and increases SR $\left[\mathrm{Ca}^{2+}\right]$ and hence also the release and leak current.

Extraction of more detailed information is based on the response surfaces. They provide an approximation for the dependency of each biomarker on the varied parameters. We use them to draw contour plots for biomarker values in dependency on parameter values (Fig. 5). The values of the parameters not varied in these plots are listed in Table 1 (fourth column) and Tables S2-S9.

The strong influence of $g_{\mathrm{LCC}}$ on the AP biomarkers motivates the focus mainly on this parameter and how its change might be compensated for by a change of another parameter. Fig. 5 depicts biomarker values computed in the $g_{\mathrm{LCC}}-V_{\mathrm{P}, \max }$ plane. The iso lines for the upper and lower parameter values of the literature ranges are color coded in green and orange, respectively. From Fig. 5, we can read off how coordinated parameter changes can maintain important biomarkers as, for instance, APD90. Interestingly, the contours of iso-APD90 and isosystolic $\left[\mathrm{Ca}^{2+}\right]$ are similar, and a coordinated change of SERCA uptake and LCC current along them could maintain approximately both but would affect mean diastolic $\left[\mathrm{Ca}^{2+}\right]$.

\section{Sensitivity analysis and response surfaces of spark biomarkers}

Fig. 6 shows the sensitivities of the spark biomarkers with respect to the varied parameters. The spark rate is mainly influenced by the opening probability of the RyR $k_{\text {plus }}$ and the RyR permeability $g_{\text {RyR }}$. Surprisingly, the full duration at half maximum (FDHM) is only weakly affected by all five parameters. Peak calcium strongly responds to changes of the RyR permeability. The quark to spark ratio is strongly
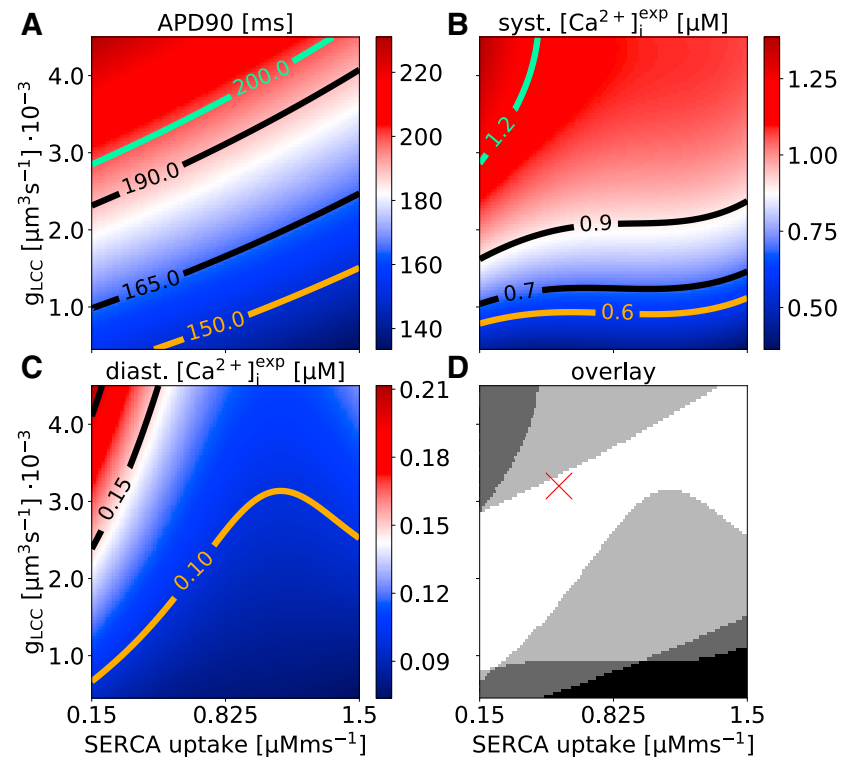

FIGURE $5(A-C)$ Contour plots for the mean values of APD90, peak systolic $\left[\mathrm{Ca}^{2+}\right]_{i}^{\text {exp }}$, and mean diastolic $\left[\mathrm{Ca}^{2+}\right]_{i}^{\exp }$ in dependence on LCC permeability $g_{\mathrm{LCC}}$ and maximal SERCA uptake $V_{\mathrm{P}, \max }$ with the values for $k_{\text {plus }}$, $k_{\text {close }}$, and $g_{\text {RyR }}$ fixed to the values in Table 1 (fourth column). The iso lines for the upper and lower parameter values of the literature ranges are color coded in green and orange, respectively. (D) Shown are the contours limiting the literature value ranges for all three biomarkers in a single plot. The white area outlines the parameter region for $g_{\mathrm{LCC}}$ and $V_{\mathrm{P}, \max }$ within which all three AP biomarkers are within the literature ranges. The red mark indicates the parameter set listed in Table 1 (fourth column), meeting also the spark biomarker requirements. To see this figure in color, go online.

negatively affected by changes in $k_{\text {plus }}$ and $g_{\text {RyR }}$ and positively by $k_{\text {close }}$.

The response surfaces of FDHM, spark rate, and $\left[\mathrm{Ca}^{2+}\right]_{i}^{\exp }$ are depicted in Fig. 7. $\left[\mathrm{Ca}^{2+}\right]_{i}^{\text {exp }}$ was calculated from $\mathrm{Ca}^{2+}-$ bound dye buffer, as in experimental analyses, by using Eq. 3. Although this inferred $\left[\mathrm{Ca}^{2+}\right]$ has a reasonable accuracy for the mean $\left[\mathrm{Ca}^{2+}\right]$ during APs (see Fig. 3), our simulations suggest that it fails for the quantification of spark peak $\left[\mathrm{Ca}^{2+}\right]_{\mathrm{i}}$ (compare Figs. S4 and S7). Whereas the true peak $\left[\mathrm{Ca}^{2+}\right]_{\mathrm{i}}$ reaches values of more than $20 \mu \mathrm{M}$ for single sparks, the inferred experimental concentration $\left[\mathrm{Ca}^{2+}\right]_{i}^{\exp }$ only reaches values slightly above $1 \mu \mathrm{M}$. A similar discrepancy occurs for the spark FDHM. Although the true underlying spark events appear to be short release events with a duration of 5-15 ms, the FDHM for the inferred $\left[\mathrm{Ca}^{2+}\right]_{i}^{\exp }$ is by factor of $\sim 2$ longer.

\section{Functional consequences of geometrical properties of RyR clusters}

We start with comparing two different models for RyR placement in clusters. The first model assumes a regular arrangement with equidistant spacing $(40 \mathrm{~nm})$ of RyRs on a regular grid, whereas the second one assumes irregular clustering properties on the basis of the measurements of 


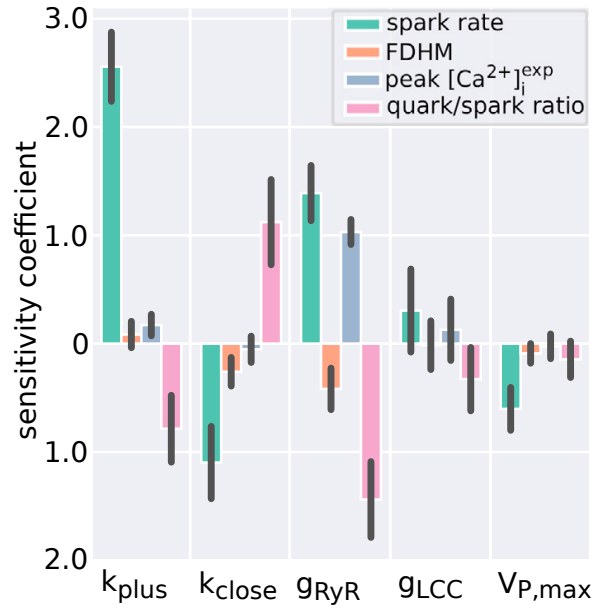

FIGURE 6 Sensitivity values for spark rate, mean peak $\left[\mathrm{Ca}^{2+}\right]$, spark FDHM, and quark to spark ratio. Black bars represent the SD for the sensitivity coefficients obtained from Bayesian inference and indicate the uncertainty in the estimates. To see this figure in color, go online.

Jayasinghe et al. (17) as described before. We use the suggested values of the parameter set in Table 1 and performed 10 simulations for each placement model. Channel numbers for the individual CRUs are drawn from the same distribution (Eq. S12) for both groups. Differences between the in-
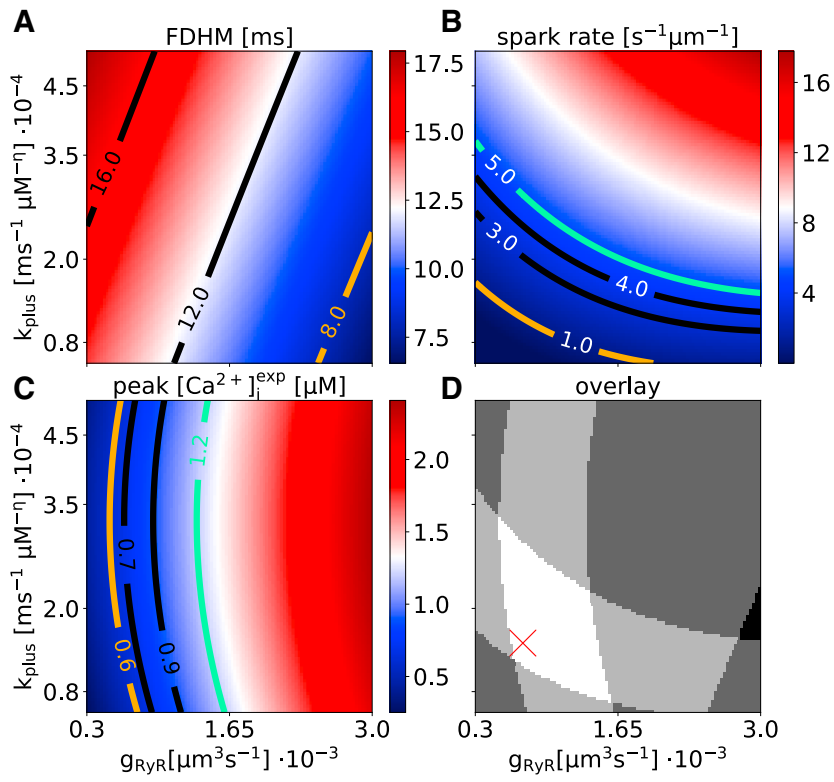

FIGURE $7(A-C)$ Contour plots for the mean values of FDHM, spark rate, and peak $\left[\mathrm{Ca}^{2+}\right]_{i}^{\text {exp }}$ inferred from Fluo-4 bound $\mathrm{Ca}^{2+}$ (Eq. 3) in dependence on the RyR permeability $g_{\mathrm{RyR}}$ and opening rate $k_{\mathrm{plus}}$ with the values for $g_{\mathrm{LCC}}, k_{\mathrm{close}}$, and $V_{\mathrm{P}, \max }$ fixed to Table 1 (fourth column). The iso lines for the upper and lower parameter values of the literature ranges are color coded in green and orange, respectively. (D) Shown are the contours limiting the literature value ranges for all three biomarkers in a single plot. The white area outlines the parameter region for $g_{\text {RyR }}$ and $k_{\text {plus }}$ within which all three spark biomarkers are within the literature ranges. The red mark indicates the parameter set listed in Table 1 (fourth column), meeting also the AP biomarker requirements. To see this figure in color, go online. dividual simulations even within one placement model group arise from the randomness of channel numbers in CRUs and their placement. We find clear differences of biomarker values between the two placement models (Figs. 8 and 9). Hence, the dyadic substructure clearly affects cellular responses.

Geometric effects within the placement model by Jayasinghe et al. are shown in Fig. 10. A variety of channel configurations has been generated by sampling from the placement model distributions and additionally varying the distribution parameters. Although channel configurations have been characterized successfully by the adjacency matrix $(22,26)$, we are looking here for a simpler approach. We characterized channel configurations by a variety of measures (average nearest- and four nearestneighbor distance, area per channel determined by convex hull, and mean occupancy (see Fig. S5)) and found mean occupancy to show the strongest correlation with biomarker values. Mean occupancy is 1 if all RyRs are far apart and 0 if all RyRs are in the same spot (Fig. S5).

APD90, peak systolic $\left[\mathrm{Ca}^{2+}\right]_{i}^{\text {exp }}$, and diastolic $\left[\mathrm{Ca}^{2+}\right]_{i}^{\text {exp }}$ decrease with increasing mean occupancy (Fig. 10). This concerted decrease reflects the correlation between these values found in the contour plots in Fig. 5, too. All three trends are in line with the general picture of decreased $\mathrm{Ca}^{2+}$ release due to increased mean occupancy. Hence, we find effects of dyadic substructure also within one placement concept.
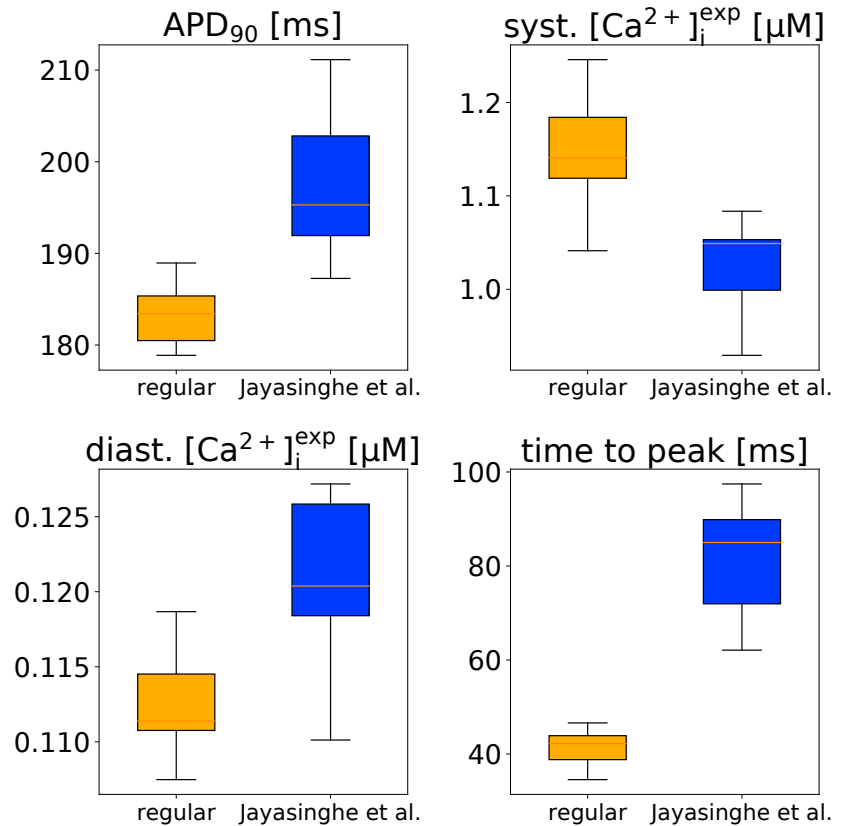

FIGURE 8 AP biomarker values APD90, systolic and diastolic $\left[\mathrm{Ca}^{2+}\right]_{i}^{\text {exp }}$, and time to peak from simulations with two different RyR placement models, which are explained in the text. The boxplots are standard box and whisker diagrams. To see this figure in color, go online. 

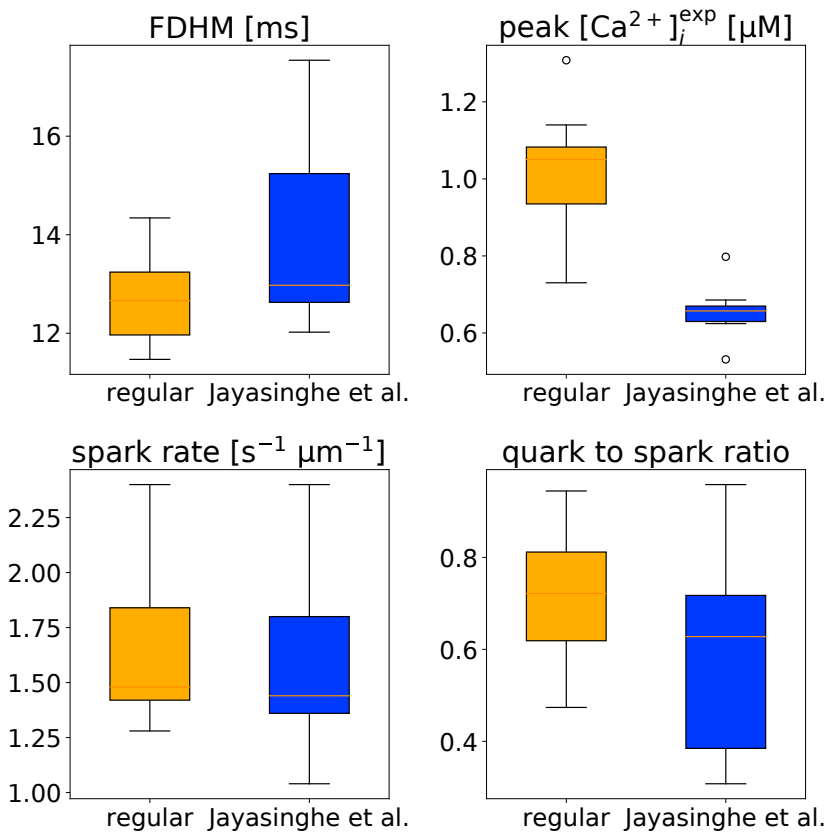

FIGURE 9 Spark biomarker values FDHM, peak $\left[\mathrm{Ca}^{2+}\right]_{i}^{\text {exp }}$, spark rate, and quark to spark ratio from simulations with two different RyR placement models. The boxplots are standard box and whisker diagrams. To see this figure in color, go online.

The regular placement exhibits smaller APD90, larger peak systolic $\left[\mathrm{Ca}^{2+}\right]_{i}^{\text {exp }}$, and shorter time to peak (Fig. 10). We assume that this is caused by the relation between LCC placement and the overall cluster shape. LCC locations were chosen according to the same rules described above for both placement methods. The Jayasinghe placement produces elongated clusters, and the regular placement produces quadratic clusters. Hence, the average RyR distance to the closest LCC is smaller with the regular placement than with the Jayasinghe placement, which entails stronger LCC-RyR coupling. This stronger and earlier $\mathrm{Ca}^{2+}$ release causes faster $\mathrm{Ca}^{2+}$-dependent inhibition of LCCs and thus shorter APD.

The strength of the coupling of RyRs by $\mathrm{Ca}^{2+}$ diffusion decreases with increasing mean occupancy. The averages of spark biomarker values depend in the expected manner on occupancy as the slopes of the linear regressions show (Fig. 11). FDHM increases with increasing mean occupancy, reflecting the known phenomenon of slower termination of sparks with weaker spatial coupling of RyRs. Correspondingly, peak systolic $\left[\mathrm{Ca}^{2+}\right]_{i}^{\text {exp }}$ and spark rate decreases with increasing mean occupancy. Large quark to spark ratios were found with weaker RyR coupling at large mean occupancy only. The spark biomarker values exhibit much stronger fluctuations than the AP simulations. The results with regular placement fit into the relation on mean occupancy.

Only the specific realizations of channel numbers in the individual CRUs vary between the individual simulations with the regular placement. Simulations with the Jayasinghe
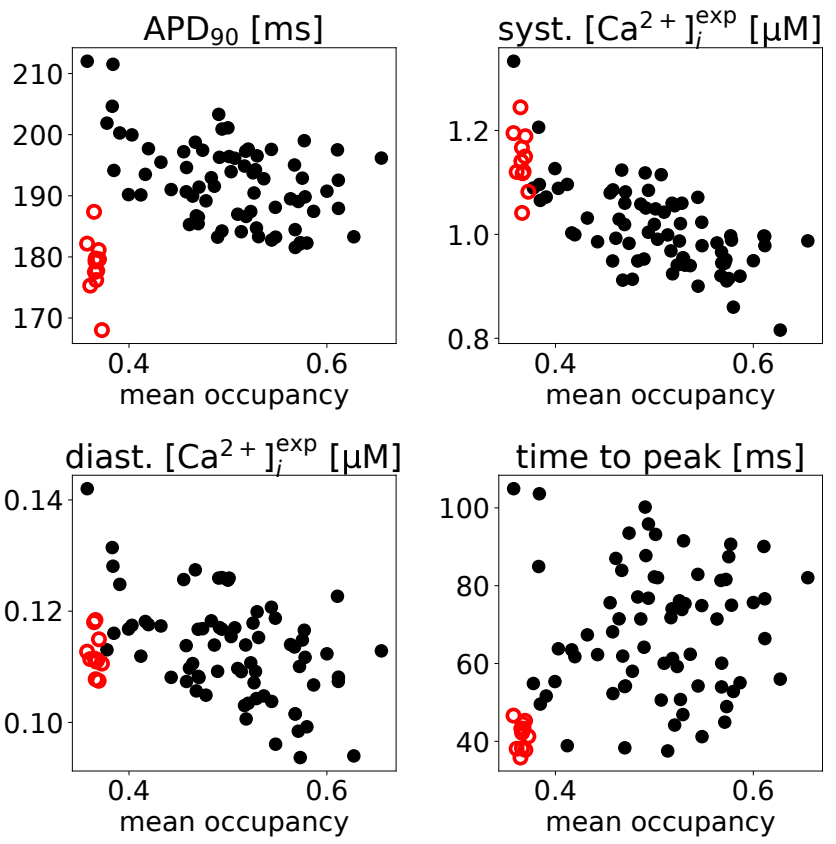

FIGURE 10 The relation between mean occupancy, as described in Fig. S5 and averaged over all CRUs, and the biomarkers APD90, systolic and diastolic $\left[\mathrm{Ca}^{2+}\right]_{i}^{\text {exp }}$, and time to peak. Each dot corresponds to one simulation. Black dots mark Jayasinghe placement, and red circles mark regular placement. The Jayasinghe placement with the measured parameters has a mean occupancy of $\sim 0.49$. The slopes of a linear regression for the Jayasinghe placement are as follows: APD90 $-38 \mathrm{~ms}$, systolic $\left[\mathrm{Ca}^{2+}\right]_{i}^{\exp }$ $-0.68 \mu \mathrm{M}$, diastolic $\left[\mathrm{Ca}^{2+}\right]_{i}^{\text {exp }}-0.056 \mu \mathrm{M}$, and time to peak $13 \mathrm{~ms}$. To see this figure in color, go online.

placement are distinguished by both channel number realizations and specific placement. Hence, comparing the scatter of the biomarker value results with regular placement (red circles) with the Jayasinghe placement (black dots) in Figs. 10 and 11 provides an idea of how much of the variability is due to the randomness of channel numbers per CRU. The variability due to channel number randomness is comparable to the total variability for the spark biomarkers FDHM and rate (Fig. 11). Jayasinghe placement increases the quark to spark ratio variability with increasing mean occupancy because coupling between channels becomes weaker. Surprisingly, the regular placement has a larger variability of the peak $\left[\mathrm{Ca}^{2+}\right]_{i}^{\exp }$ than the Jayasinghe placement because it exhibits also very large values. The same comparison for the AP simulations suggests variability of peak systolic $\left[\mathrm{Ca}^{2+}\right]_{i}^{\exp }$ to result mainly from channel number variability. Variability of APD90, diastolic $\left[\mathrm{Ca}^{2+}\right]_{i}^{\text {exp }}$, and time to peak increase substantially because of the Jayasinghe placement.

\section{DISCUSSION}

Parameterization of detailed multiscale models faces the problem of large computational costs required for 

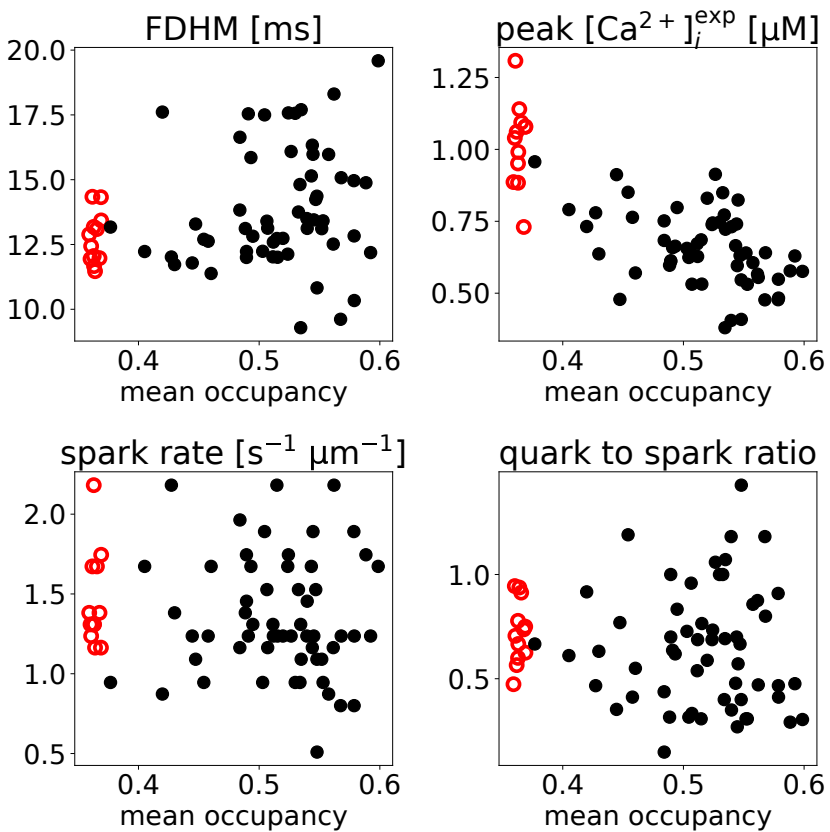

FIGURE 11 The relation between mean occupancy, as described in Fig. S5 and averaged over all CRUs, and the biomarkers FDHM, peak $\left[\mathrm{Ca}^{2+}\right]_{i}^{\text {exp }}$, spark rate, and quark to spark ratio. Each dot corresponds to one simulation. Black dots mark Jayasinghe placement, and red circles mark regular placement. The Jayasinghe placement with the measured parameters has a mean occupancy of $\sim 0.49$. The slopes of a linear regression for the Jayasinghe placement are as follows: FDHM $7.42 \mathrm{~ms}$, peak $\left[\mathrm{Ca}^{2+}\right]_{i}^{\exp }$ $-0.88 \mu \mathrm{M}$, spark rate $-0.37 \mathrm{~s}^{-1}$, and quark to spark ratio -0.11 . To see this figure in color, go online.

simulations, which often prevents systematic parameter searches. These models require large efforts to reduce compute time and methods concluding parameter dependencies from a minimum of simulations. We reported our efforts to speed up simulations in previous studies $(1,6)$. Here, we present a parameterization of the model to experimental data. To that end, we identified ranges of altogether 11 biomarker values from literature, four of them were fulfilled by model output, mainly based on previous work by Mahajan et al. (36), and seven of them were affected by our spatially detailed approach and had to be met by our parameterization procedure. The multiscale set up of our model allowed for detailed channel placement inside dyadic clefts, according to measurements in Jayasinghe et al. (17). Accordingly, we focused here on parameters that are crucial for the $\mathrm{Ca}^{2+}$ handling in the microscopic domain of the dyadic cleft: the opening and closing rates of RyRs $k_{\text {plus }}$ and $k_{\text {close }}$, the RyR permeability $g_{\text {RyR }}$, the LCC permeability $g_{\mathrm{LCC}}$, and the strength of SERCA uptake $V_{\mathrm{P}, \max }$.

In the case of AP simulations, LCC permeability $g_{\mathrm{LCC}}$ variation has the strongest impact on cell behavior, as illustrated by the sensitivities in Fig. 4. The response surfaces in Fig. 5 illustrate the strong correlation between APD and the systolic $\left[\mathrm{Ca}^{2+}\right]_{i}$ values. As an example of a control strategy suggested by response surfaces, we note that a coordinated change of $g_{\mathrm{LCC}}$ and $V_{\mathrm{P}, \max }$ can approximately maintain APD and systolic $\left[\mathrm{Ca}^{2+}\right]_{\mathrm{i}}$ while lowering diastolic $\left[\mathrm{Ca}^{2+}\right]_{i}$, if the initial APD is in the lower range of the literature values. That would be a strategy to decrease the propensity for diastolic triggered events while maintaining contraction.

The spark simulations show that $k_{\text {plus }}, k_{\text {close }}$, and $g_{\mathrm{RyR}}$ affect the spark rate in the way we expected: $k_{\text {plus }}$ and $g_{\text {RyR }}$ positively, $k_{\text {close }}$ negatively. Their effect on the quark to spark ratio can be comprehended by considering the probability that the first open RyR does open another one, thus turning a quark into a spark. That probability increases with $k_{\text {plus }}$ and $g_{\text {RyR }}$ and therefore reduces the quark to spark ratio and vice versa with $k_{\text {close }}$. The weak effect of all five parameters on the FDHM is surprising at a first glance; however, it agrees with the results by Cannell et al. (25) for the latency of induction decay.

The Jayasinghe placement affects the mean and SD of the AP biomarker values and mean and/or the SD of the spark biomarker values we have investigated. Hence, subdyadic structure matters. Figs. 8, 9, 10, and 11 report the effect of the Jayasinghe placement compared to the regular placement. These effects are due to cluster shape and channel density. The elongated shape of the clusters generated by the Jayasinghe placement weakens LCC-RyR coupling (compared to the regular placement), which increases time to peak and APD90 and affects indirectly diastolic and peak systolic $\left[\mathrm{Ca}^{2+}\right]_{i}^{\text {exp }}$. Mean occupancy is related to density and mean channel distance. In principle, the SD of channel distances could also have an effect on CRU dynamics by generating highly coupled subclusters at large SD. However, that is not supported by our simulations.

We found the effects of channel placement, which might be affected by specifics of our cleft model. We assume a fraction of $50 \%$ of total $\mathrm{Ca}^{2+}$ to be buffered by mobile buffers based on estimates from (26). This estimate assumes ATP $\left(\mathrm{K}_{D} \approx 200 \mu \mathrm{M}(26)\right)$ to be the dominating mobile buffer at $\mathrm{Ca}^{2+}$ concentrations occurring in the dyadic space. ATP levels may change to a degree affecting dyadic buffering in pathological states; however, this was not in the scope of this study $(47,48)$. We use an effective diffusion coefficient of $100 \mu \mathrm{m}^{2} \mathrm{~s}^{-1}$ inside the cleft as well as a quasistatic approximation for the concentration profiles (see Supporting Materials and Methods; (1,6,32,33)). Coupling of RyRs upon the opening of a channel with dynamic concentration profiles is initially weaker than with quasistatic profiles. Geometric effects are more important with weak spatial coupling. Hence, we assume that dynamic profiles would slightly amplify them. The timescales of the cytosolic concentration around the cleft space dominate the dynamics upon closing. They are captured by our model. The diffusion coefficient in the dyadic space has not been measured, and we informed our model on the basis of other modeling studies $(25,26,49)$. Because we are using an effective value, it is rather at the upper end of the currently accepted range. 
Cosi et al.

In summary, we may rather underestimate than overestimate the geometrical effects.

In this study, we used multiscale modeling in two ways complementary to experimental studies. We observed local dynamics and cellular behavior simultaneously, and we modified subdyadic structures, which cannot be modified by experimental means. That provided insight into structure-function relations across multiple scales. Our results suggest that both the mean occupancy and the overall cluster shape affect APs and cytosolic $\mathrm{Ca}^{2+}$ transients.

\section{SUPPORTING MATERIAL}

Supporting Material can be found online at https://doi.org/10.1016/j.bpj. 2019.09.023.

\section{AUTHOR CONTRIBUTIONS}

M.F., W.G., F.G.C., N.C., U.P., and S.L. designed the research. W.G., F.G.C., and W.N. performed the research. W.G. and F.G.C. analyzed the data. M.F., F.G.C., W.G., N.C., U.P., and S.L. wrote the manuscript.

\section{ACKNOWLEDGMENTS}

N.C. acknowledges support from the Science and Engineering Research Board, Department of Science and Technology, India (EMR/2017/000664 and MTR/2017/00 0598). F.G.C., U.P. and S.L. acknowledge support from the German Research Foundation (DFG) via SFB 937 (project A18). S.L. acknowledges support through the German Center for Cardiovascular Research, partner site Göttingen. F.G.C. acknowledges support by the Physics to Medicine program and the Göttingen Graduate Center for Neurosciences, Biophysics, and Molecular Biosciences.

\section{REFERENCES}

1. Vierheller, J., W. Neubert, ..., N. Chamakuri. 2015. A multiscale computational model of spatially resolved calcium cycling in cardiac myocytes: from detailed cleft dynamics to the whole cell concentration profiles. Front. Physiol. 6:255.

2. Rajagopal, V., G. Bass, ..., C. Soeller. 2015. Examination of the effects of heterogeneous organization of RyR clusters, myofibrils and mitochondria on $\mathrm{Ca}^{2+}$ release patterns in cardiomyocytes. PLoS Comput. Biol. 11:e1004417

3. Hatano, A., J. Okada, ..., S. Sugiura. 2015. Distinct functional roles of cardiac mitochondrial subpopulations revealed by a 3D simulation model. Biophys. J. 108:2732-2739.

4. Song, Z., Z. Qu, and A. Karma. 2017. Stochastic initiation and termination of calcium-mediated triggered activity in cardiac myocytes Proc. Natl. Acad. Sci. USA. 114:E270-E279.

5. Colman, M. A., C. Pinali, ..., A. Kitmitto. 2017. A computational model of spatio-temporal cardiac intracellular calcium handling with realistic structure and spatial flux distribution from sarcoplasmic reticulum and t-tubule reconstructions. PLoS Comput. Biol. 13:e1005714.

6. Chamakuri, N., W. Neubert, ..., M. Falcke. 2018. Multiscale modeling and numerical simulation of calcium cycling in cardiac myocytes. SIAM Multiscale Modeling \& Simulation. 16:1115-1145.

7. Mirams, G. R., P. Pathmanathan, ..., R. H. Clayton. 2016. Uncertainty and variability in computational and mathematical models of cardiac physiology. J. Physiol. 594:6833-6847.
8. Johnstone, R. H., E. T. Y. Chang, ..., G. R. Mirams. 2016. Uncertainty and variability in models of the cardiac action potential: can we build trustworthy models? J. Mol. Cell. Cardiol. 96:49-62.

9. Krogh-Madsen, T., A. F. Jacobson, ..., D. J. Christini. 2017. Global optimization of ventricular myocyte model to multi-variable objective improves predictions of drug-induced Torsades de Pointes. Front. Physiol. 8:1059.

10. Vagos, M. R., H. Arevalo, ..., M. M. Maleckar. 2017. A computational framework for testing arrhythmia marker sensitivities to model parameters in functionally calibrated populations of atrial cells. Chaos. 27:093941.

11. Chang, E. T., M. Strong, and R. H. Clayton. 2015. Bayesian sensitivity analysis of a cardiac cell model using a Gaussian process emulator. PLoS One. 10:e130252.

12. Renardy, M., T. M. Yi, ..., C. S. Chou. 2018. Parameter uncertainty quantification using surrogate models applied to a spatial model of yeast mating polarization. PLoS Comput. Biol. 14:e1006181.

13. Xiu, D., and G. E. Karniadakis. 2002. The Wiener-Askey polynomial chaos for stochastic differential equations. SIAM J. Sci. Comput. 24:619-644.

14. Macquaide, N., H. T. Tuan, ..., K. R. Sipido. 2015. Ryanodine receptor cluster fragmentation and redistribution in persistent atrial fibrillation enhance calcium release. Cardiovasc. Res. 108:387-398.

15. Xiu, D. 2009. Fast numerical methods for stochastic computations: a review. Commun. Comput. Phys. 5:242-272.

16. Jones, P. P., N. MacQuaide, and W. E. Louch. 2018. Dyadic plasticity in cardiomyocytes. Front. Physiol. 9:1773.

17. Jayasinghe, I., A. H. Clowsley, ..., C. Soeller. 2018. True molecular scale visualization of variable clustering properties of ryanodine receptors. Cell Rep. 22:557-567.

18. Baddeley, D., I. D. Jayasinghe, ..., C. Soeller. 2009. Optical singlechannel resolution imaging of the ryanodine receptor distribution in rat cardiac myocytes. Proc. Natl. Acad. Sci. USA. 106:2227522280 .

19. Jayasinghe, I. D., M. B. Cannell, and C. Soeller. 2009. Organization of ryanodine receptors, transverse tubules, and sodium-calcium exchanger in rat myocytes. Biophys. J. 97:2664-2673.

20. Asghari, P., D. R. Scriven, ..., E. D. Moore. 2014. Nonuniform and variable arrangements of ryanodine receptors within mammalian ventricular couplons. Circ. Res. 115:252-262.

21. Hou, Y., I. Jayasinghe, ..., C. Soeller. 2015. Nanoscale analysis of ryanodine receptor clusters in dyadic couplings of rat cardiac myocytes. J. Mol. Cell. Cardiol. 80:45-55

22. Walker, M. A., T. Kohl, ..., R. L. Winslow. 2015. On the adjacency matrix of RyR2 cluster structures. PLoS Comput. Biol. 11:e1004521.

23. Galice, S., Y. Xie, ..., D. M. Bers. 2018. Size matters: ryanodine receptor cluster size affects arrhythmogenic sarcoplasmic reticulum calcium release. J. Am. Heart Assoc. 7:e008724.

24. Shen, X., J. van den Brink, ..., W. E. Louch. 2019. 3D dSTORM imaging reveals novel detail of ryanodine receptor localization in rat cardiac myocytes. J. Physiol. 597:399-418.

25. Cannell, M. B., C. H. Kong, ..., D. R. Laver. 2013. Control of sarcoplasmic reticulum $\mathrm{Ca}^{2+}$ release by stochastic RyR gating within a 3D model of the cardiac dyad and importance of induction decay for CICR termination. Biophys. J. 104:2149-2159.

26. Walker, M. A., G. S. B. Williams, ..., R. L. Winslow. 2014. Superresolution modeling of calcium release in the heart. Biophys. J. 107:30183029

27. Bastian, P., M. Blatt, A. Dedner, C. Engwer, R. Klöfkorn, R. Kornhuber, M. Ohlberger, and O. Sander. 2008. A generic grid interface for parallel and adaptive scientific computing. Part II: implementation and tests in DUNE. Computing. 82:121-138.

28. Bastian, P., M. Blatt, A. Dedner, N.-A. Dreier, C. Engwer, R. Fritze, C Gräser, D. Kempf, R. Klöfkorn, M. Ohlberger, and O. Sander. 2019. The DUNE framework: basic concepts and recent developments. arXiv preprint, arXiv:1909.13672. 
29. Restrepo, J. G., and A. Karma. 2009. Spatiotemporal intracellular calcium dynamics during cardiac alternans. Chaos. 19:037115.

30. Nivala, M., E. de Lange, ..., Z. Qu. 2012. Computational modeling and numerical methods for spatiotemporal calcium cycling in ventricular myocytes. Front. Physiol. 3:114.

31. Nivala, M., Z. Song, ..., Z. Qu. 2015. T-tubule disruption promotes calcium alternans in failing ventricular myocytes: mechanistic insights from computational modeling. J. Mol. Cell. Cardiol. 79:32-41.

32. Schendel, T., and M. Falcke. 2010. Efficient and detailed model of the local $\mathrm{Ca}^{2+}$ release unit in the ventricular cardiac myocyte. Genome Inform. 22:142-155.

33. Schendel, T., R. Thul, ..., M. Falcke. 2012. How does the ryanodine receptor in the ventricular myocyte wake up: by a single or by multiple open L-type $\mathrm{Ca}^{2+}$ channels? Eur. Biophys. J. 41:27-39.

34. Lanner, J. T., D. K. Georgiou, ..., S. L. Hamilton. 2010. Ryanodine receptors: structure, expression, molecular details, and function in calcium release. Cold Spring Harb. Perspect. Biol. 2:a003996.

35. Iman, R. L., J. C. Helton, and J. E. Campbell. 1981. An approach to sensitivity analysis of computer models: Part I-Introduction, input variable selection and preliminary variable assessment. J. Qual. Technol. $13: 174-183$

36. Mahajan, A., Y. Shiferaw, ..., J. N. Weiss. 2008. A rabbit ventricular action potential model replicating cardiac dynamics at rapid heart rates. Biophys. J. 94:392-410.

37. Gemmell, P., K. Burrage, ..., T. A. Quinn. 2016. Rabbit-specific computational modelling of ventricular cell electrophysiology: using populations of models to explore variability in the response to ischemia. Prog. Biophys. Mol. Biol. 121:169-184.

38. Box, G. E. 1980. Sampling and Bayes' inference in scientific modelling and robustness. J. R. Stat. Soc. Ser. A. 143:383-430.
39. Tran, D., A. Kucukelbir, ..., D. M. Blei. 2016. Edward: a library for probabilistic modeling, inference, and criticism. arXiv, arXiv:1610. 09787 https://arxiv.org/abs/1610.09787.

40. Abadi, M., P. Barham, ..., X. Zheng. 2016. Tensorflow: a system for large-scale machine learning. In 12th USENIX Symposium on Operating Systems Design and Implementation, OSDI 2016, pp. 265-283.

41. Saltelli, A., and I. M. Sobol'. 1995. Sensitivity analysis for nonlinear mathematical models: numerical experience. Matematicheskoe Modelirovanie. 7:16-28.

42. Feinberg, J., and H. P. Langtangen. 2015. Chaospy: an open source tool for designing methods of uncertainty quantification. J. Comput. Sci. $11: 46-57$

43. Sudret, B. 2008. Global sensitivity analysis using polynomial chaos expansions. Reliab. Eng. Syst. Saf. 93:964-979.

44. Williams, G. S., A. C. Chikando, ..., M. S. Jafri. 2011. Dynamics of calcium sparks and calcium leak in the heart. Biophys. J. 101:12871296.

45. Takahashi, A., P. Camacho, ..., B. Herman. 1999. Measurement of intracellular calcium. Physiol. Rev. 79:1089-1125.

46. Britton, O. J., A. Bueno-Orovio, ..., B. Rodriguez. 2013. Experimentally calibrated population of models predicts and explains intersubject variability in cardiac cellular electrophysiology. Proc. Natl. Acad. Sci. USA. 110:E2098-E2105.

47. Neubauer, S. 2007. The failing heart-an engine out of fuel. N. Engl. J. Med. 356:1140-1151.

48. Ingwall, J. 2002. ATP and the Heart. Basic Science for the Cardiologist. Springer, New York.

49. Hake, J., A. G. Edwards, ..., A. D. McCulloch. 2012. Modelling cardiac calcium sparks in a three-dimensional reconstruction of a calcium release unit. J. Physiol. 590:4403-4422. 


\subsection{Polynomial Chaos Expansion - Revisited}

After the publication of [50] I revised the method for generating a PCE surrogate model by computing the best $\alpha$-CVS pair for the PCE regression of each biomarker. I developed an optimiser, which allowed to search the best $\alpha$-CVS pair for the different maximal polynomial orders used in the PCE. The optimiser scans with a gradient descent and manually selected initial values the $\alpha$ range between 0 and 1 and looks for the penalisation factor that yields the best CVS.

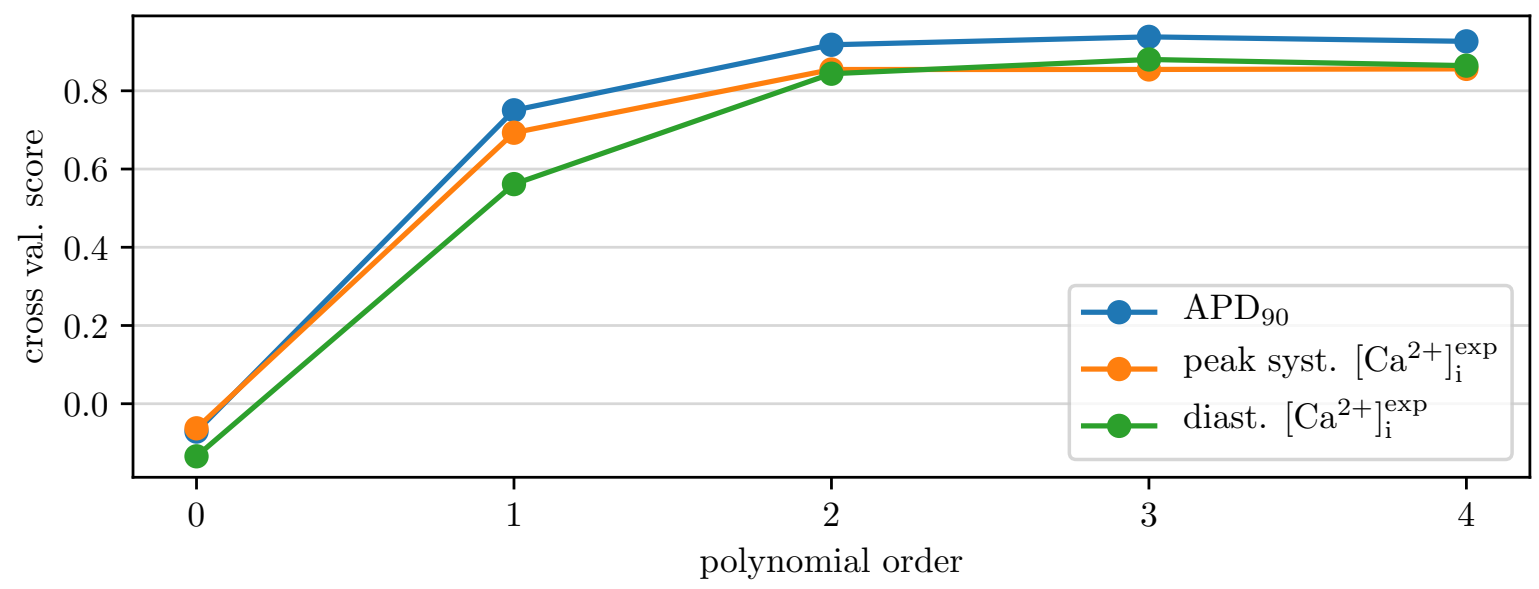

Figure 4.1 - Optimised cross validation score as function of polynomial order of the used PCE regression for the main three AP biomarkers APD $D_{90}$, peak calcium value and diastolic calcium value.

The resulting best cross validation scores for both simulation types, i.e. action potential and spark simulations, are shown as functions of the fitting polynomial order in figures 4.1 and 4.2, respectively. The first increase in the CVS from polynomials with order zero to those with order one is apparent and clear. In AP simulations (figure 4.1) the CVS keeps increasing with the polynomial order up to 3, where it saturates. This does not happen with the CVS of spark simulation biomarkers (figure 4.2), where the highest score is reached already for the first order polynomials. Nevertheless the CVS does not perceptibly decrease for higher polynomial orders. This means the PCE regression is very robust with respect to the performance of the CVS as a function of the polynomial order. It is remarkable that the biomarker computed with the highest number of available data (297 spark simulations, of which only 140 are with Fluo-4 bound calcium), i.e. the number of sparks, shows the worse cross validation score. Why this might be the case, is discussed together with the question on spark determination in section 4.2.2.

Additionally the action potential simulations perform better than the spark simulations, this might be due to higher fluctuations in the determination of the biomarkers of the latter. 


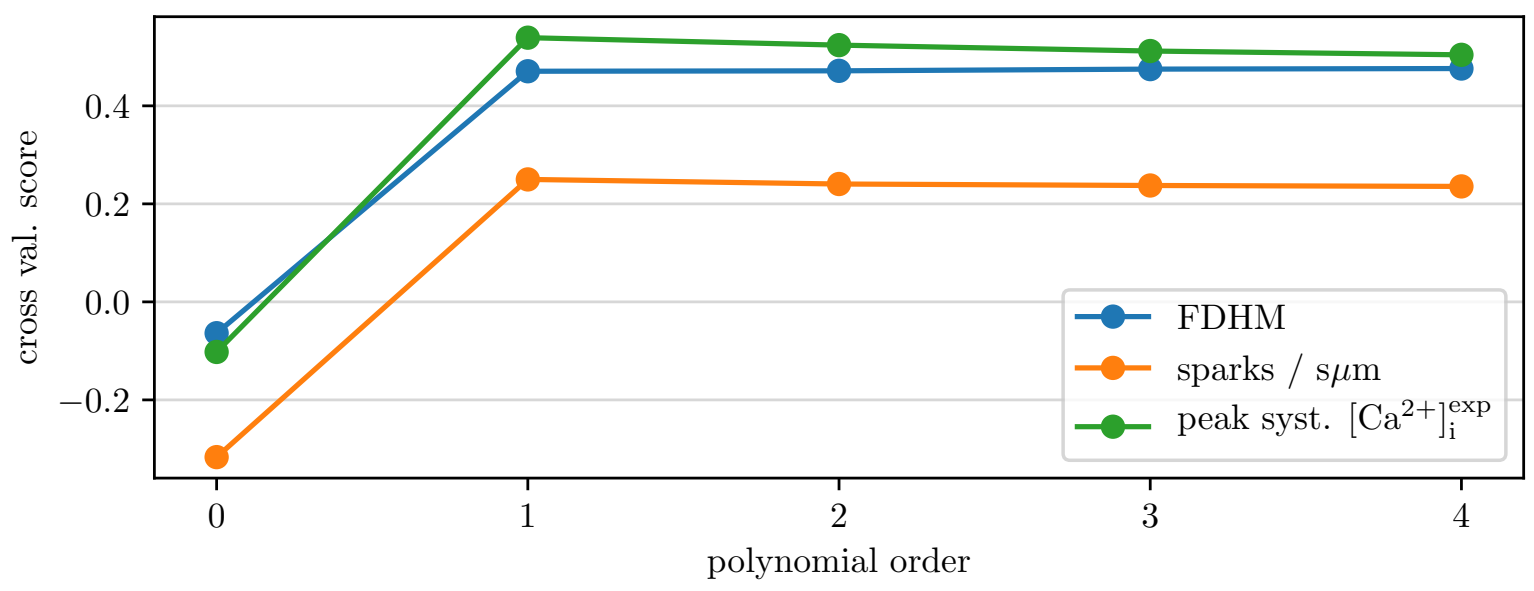

Figure 4.2 - Cross validation score from the implemented optimiser as function of polynomial order of the used PCE regression for the three main spark biomarkers FDHM, number of sparks and peak calcium value.

\subsubsection{Surrogate Model}

Employing the newly computed $\alpha$-CVSs pairs it was possible to generate a new PCE regression for the analysed biomarkers in both simulation types. For both AP and spark simulations I chose to compute the PCE regression using three as the maximal polynomial order. This is apparent for AP simulations from the trend of the CVS with respect to the polynomial order in figure 4.1, while it is not for the spark simulations. Here I still chose to use the third order Legendre polynomials, since orders one to four perform similar, so there is no harm in choosing the order 3 over e.g. 1. Further, the amount of spark data (297 simulation runs) allows for a regression with third order polynomials and is consistent with the degree chosen to fit the AP biomarkers.

With the newly computed PCE regressions now more robust surrogate models, compared to the previous ones, allow for the search of parameter regions based on given biomarker values. Phrased differently this means that a fast-run surrogate model is generated that works similarly to a look-up table for the selected biomarker. While the PCE regression outlined in [50] served the purpose of confining the parameter space, in which the quest for a parameter set yielding physiological output was undertaken, now it was improved to be employed as a fast and robust surrogate model. Here robust means that the CVS is optimised and the surrogate model best describes the complex model's output.

\section{Contours revisited}

With the optimised cross validation scores the PCE regressions for the main biomarkers shown in figure 5 and 7 of [50] can be redone. The results are depicted in figures 4.3 and 4.4 for AP and spark simulations, respectively. The figures' structure is the same as the one of figures 5 and 7 in [50] detailed in section 4.1; the colourbar in the three coloured plots (panels A-C) shows the value of the regression function in a plane 

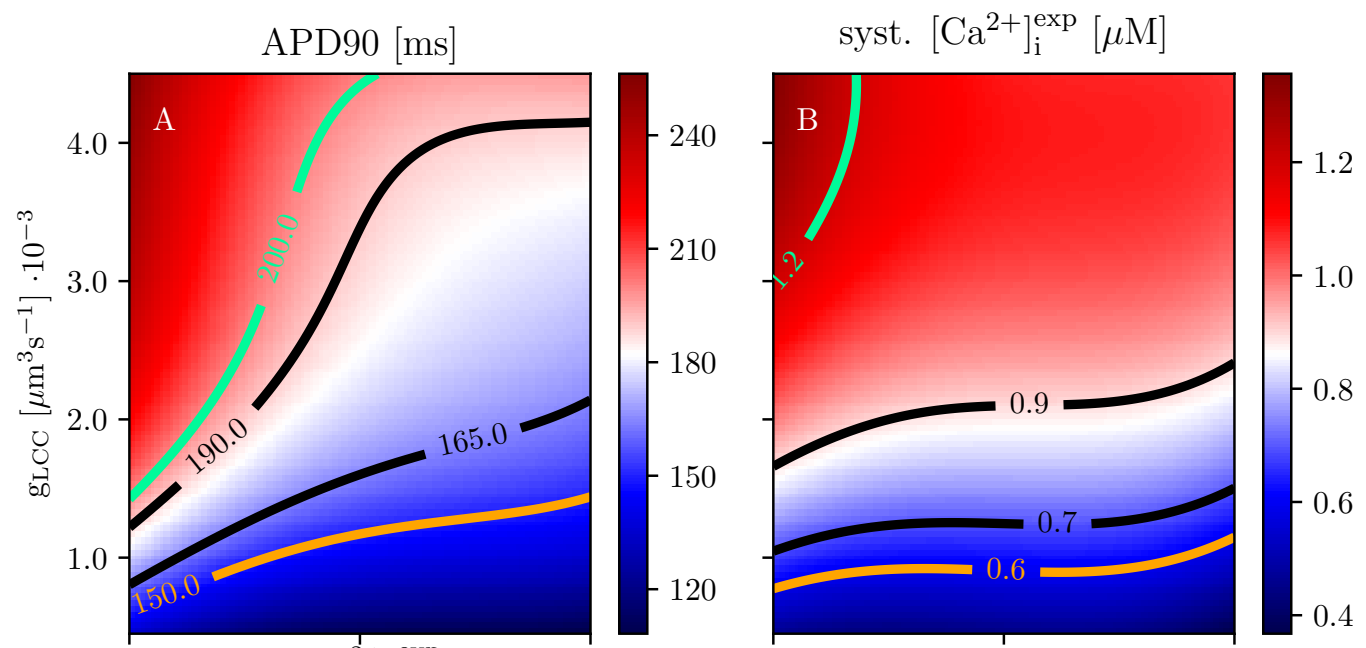

diast. $\left[\mathrm{Ca}^{2+}\right]_{\mathrm{i}}^{\exp }[\mu \mathrm{M}]$
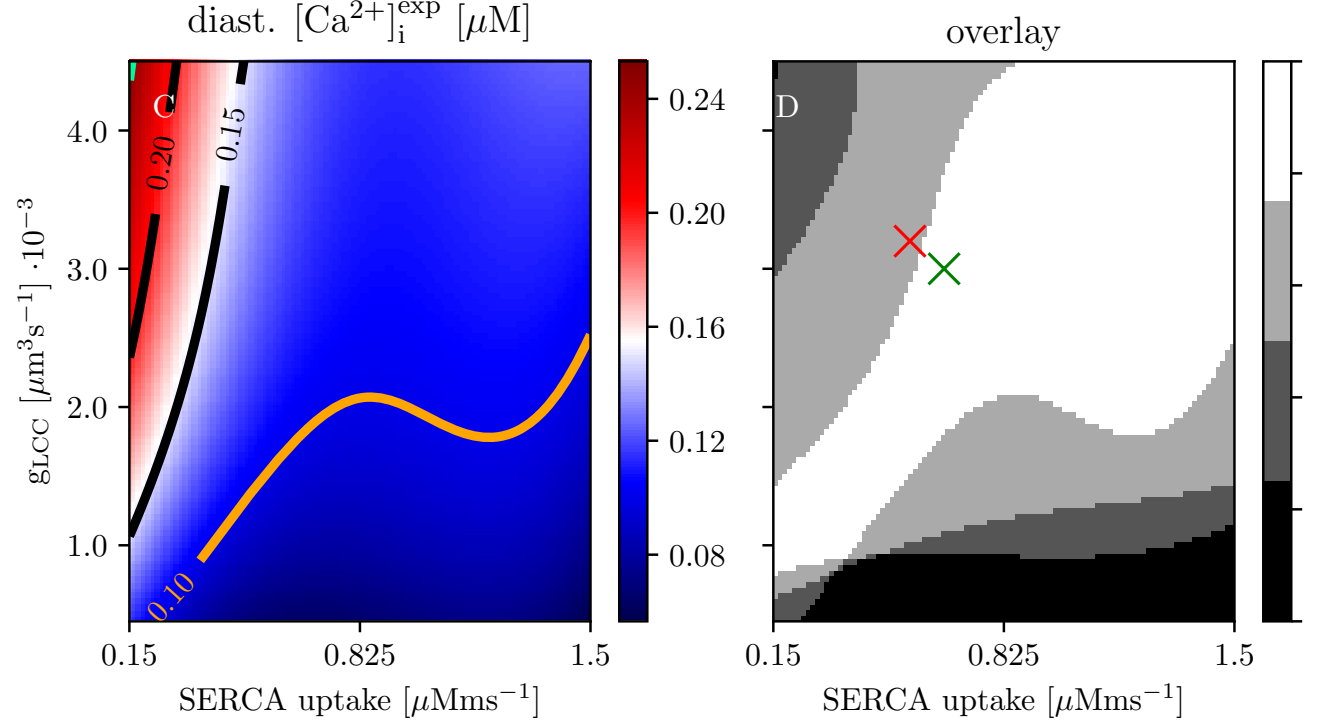

Figure 4.3 - A-C: projections of the PCE regression functions onto the $g_{\mathrm{LCC}}-V p_{\max }$ plane, with the other parameter values fixed to the selected set for the three main action potential biomarkers $A P D_{90}$, peak and diastolic calcium values. Colour-coded isolines show the upper and lower bounds of the physiological regions of the given biomarker in green and orange, respectively. D: overlay panel of the bound isolines showing the region with full physiological output in white and decreasing physiological relevance in grey scales. The green and red crosses mark the locations of the selected parameter values in [50] and with the optimised $\alpha$-CVS pair, respectively. 
spanned by two parameters (the other three are fixed), the contour lines depict some indicative values in black and the chosen physiological upper and lower limits of the depicted biomarker in green and orange, respectively.

The fourth panel D shows the overlay of the biomarker limited ranges, where white represents physiological conditions of all three depicted biomarkers. The darker the grey tone the fewer biomarkers are in the physiological region. In the overlay plot the red cross marks the parameter value of the parameter set chosen in [50], while the green one corresponds to a newly suggested one.

Comparing figure 4.3 with figure 5 in [50] it becomes clear how in the latter plot the employed penalisation $\alpha$ affects the regression. Mainly affected are the regression functions of $\mathrm{APD}_{90}$ and diastolic calcium. In the former the penalisation factor shrinks from $8.3 \times 10^{-3}$ in [50] to an optimal value of $1.4 \times 10^{-6}$. The number of coefficients that are not regularised to zero thus increases in the optimised case. The same happens for the $\alpha$ values of diastolic and peak calcium, whereat in the latter this reduction is less pronounced (from $1.6 \times 10^{-4}$ to $1.6 \times 10^{-5}$ ). Indeed peak systolic calcium is not perceivable affected by the optimisation of the CVS.

Comparison of panel D in the two figures also reveals that the chosen parameter set of [50] (red crosses) lies above the upper threshold line of $\mathrm{APD}_{90}$ sliding marginally into the region described as pathological. Here I present a refinement of the selected parameter set for the optimised regression. The green cross represents a slight shift of two parameters, namely $g_{\mathrm{LCC}}$ and $V p_{\max }$, that allows for a physiological output in both figures 4.3 and 4.4. The change in the named parameter values is kept

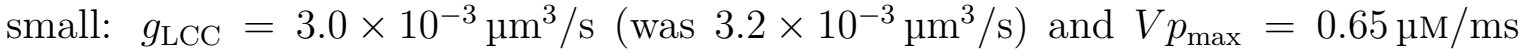
(was $0.55 \mu \mathrm{M} / \mathrm{ms}$ ). The other three parameters $k_{\text {plus }}, k_{\text {close }}$ and $g_{\text {RyR }}$, kept unchanged. This shift represents only a minor refinement that shifts the selected parameter set towards the white and thus physiological region.

The boundaries shown in the contour plots 4.3 and 4.4 and in figures 5 and 7 of [50] are moreover not sharp. The fluctuations from the stochasticity of cleftdyn render them fuzzy. This can be seen well in figures 10 and 11 of [50] where further simulations with the selected parameter set sometimes provided biomarker values beyond the chosen boundaries. Regardless of this consideration, both selected parameter sets are valid for cleftdyn for the generation of physiological relevant output as figures 10 and 11 of [50] confirm. The new proposed parameter set however better matches the optimised PCE regression function.

When looking at the differences between figures 4.4 and 7 in [50], of special interest is the projection of the regression function of the spark rate in panel B. The difference between the two representations is not very pronounced. The optimal penalisation $\alpha$ is stronger here (was $1.0 \times 10^{-4}$ and became $1.4 \times 10^{-3}$ ), but yields a better CVS (was 0.11 and became 0.24 ), meaning that in this case reducing the number of coefficients to be determined results in an improved regression. This fact confirms the robustness of the optimisation procedure. For the FDHM in panel A something similar to $\mathrm{APD}_{90}$ and diastolic calcium in the case of the AP biomarkers happens; i.e. the penalisation used for the regression in [50] might have been too strong (for FDHM $\alpha$ was $1.3 \times 10^{-3}$ 

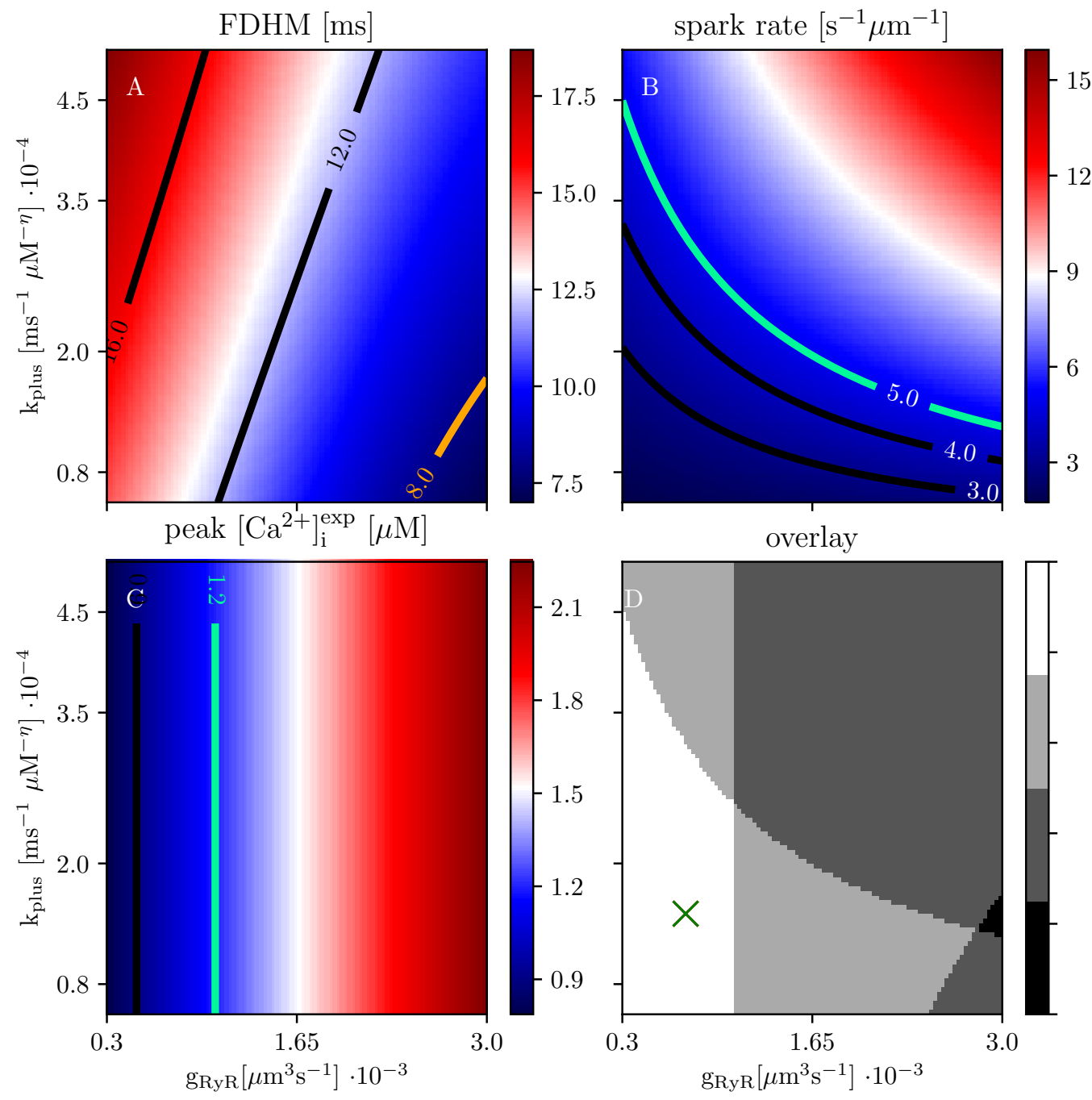

Figure $4.4-A-C$ : projections of the PCE regression functions onto the $k_{\text {plus }}-g_{\mathrm{LCC}}$ plane, with the other parameter values fixed to the selected set for the three main spark biomarkers FDHM, spark rate and peak calcium values. Colour-coded isolines show the upper and lower bounds of the given biomarker in green and orange, respectively. D: overlay panel of the bound isolines showing the region with full physiological output in white and decreasing physiological relevance in grey scales. The green cross marks the location of the newly selected parameter set. 
and becomes $7.1 \times 10^{-4}$ in optimised case) yielding a slightly modified fit. For the peak calcium concentration in panel $\mathrm{C}$ the opposite occurs; the optimal CVS enforces a higher penalisation factor $\left(\alpha=1.0 \times 10^{-3}\right.$ in [50] and $\alpha=0.016$ in the optimised case) that reduces the number of coefficients to be considered in the PCE regression. Finally in panel D the overlay plot shows the parameter region (in white) for which physiological output can be generated for the three biomarkers. In figure 4.4 the two crosses coincide (only the green one is shown), because the refinement in parameter values from the red to the green cross (shown in figure 4.3 ) does not affect $k_{\text {plus }}$ and $g_{\mathrm{LCC}}$.

\section{Implications of optimisation and meaning of a surrogate model}

Figures 4.3 and 4.4 show an improved surrogate model compared to figures 5 and 7 in [50] highlighting the importance of optimising the penalisation factor $\alpha$ and the cross validation score. The PCE regression employed in [50] served the purpose of parameter selection and since the borders of the region declared as physiological are not sharp, the parameter set determined in that context is reliable and perfectly suited for calibrating the model's input parameters. Further the similarities between the PCE regression functions of action potential simulations underline the robustness of this simulation protocol with respect to the considered biomarkers.

The shift of the selected parameter set depicted as a red cross in figure 4.3 panel D slightly into a pathological region explains the crossing visible in figures 8 and 10 of [50] of the upper bound of $\mathrm{APD}_{90}$ of the subsequent simulation runs. Nevertheless this effect is not dramatic and inside the tolerated fluctuations given by the inherent stochasticity of the model ultimately supporting the relevance of the parameter set selected in [50]. With the new parameter set we can expect a small shift of the biomarker output towards the physiological region, especially for $\mathrm{APD}_{90}$, to slightly lower values.

The optimisation in the case of spark simulations resulted in lower CVSs compared to AP simulations (see figures 4.2 and 4.1). The PCE regressions (figure 4.4) differ perceptibly from the ones in figure 7 of [50] indicating a less reliable biomarker set. This could be inherent to both, the choice of biomarkers to be analysed as well as to the definition of the biomarkers themselves. Additionally, the enhanced effect of stochasticity in spark simulations could further explain these differences. For a detailed discussion on the last two points, please see the following section 4.2.2.

Further simulations done in [50] and conducted with the parameter set selected there, i.e. the biomarker distributions for varying channel placements in figures 8-11, show that the output lies within physiological ranges. This fact retrospectively confirms the choice of the parameter set done in [50]. The outliers can be ascribed to the system's stochasticity and are therefore within the acceptable fluctuations of a model describing a biological system.

Considering the high computational demand of cleftdyn (see section 2.2.5 for details), the generation of a robust surrogate model from some only hundred simulation runs is a valuable tool for the analysis of further model features. In line with what has been done with other meta models $[51-53,55]$ it is now possible to perform fast 
analysing simulations with the surrogate model, providing a detailed overview of the impact of parameter variations on biomarkers of interest.

\subsubsection{On the variable nature of spark simulations}

In the course of this thesis we often compared action potential and spark simulations emphasising their differences and noting a less consistent and robust output in the latter. This point has to be discussed in order to embed the two types of simulations in a bigger picture and to assess their relevance, which I am doing in the following.
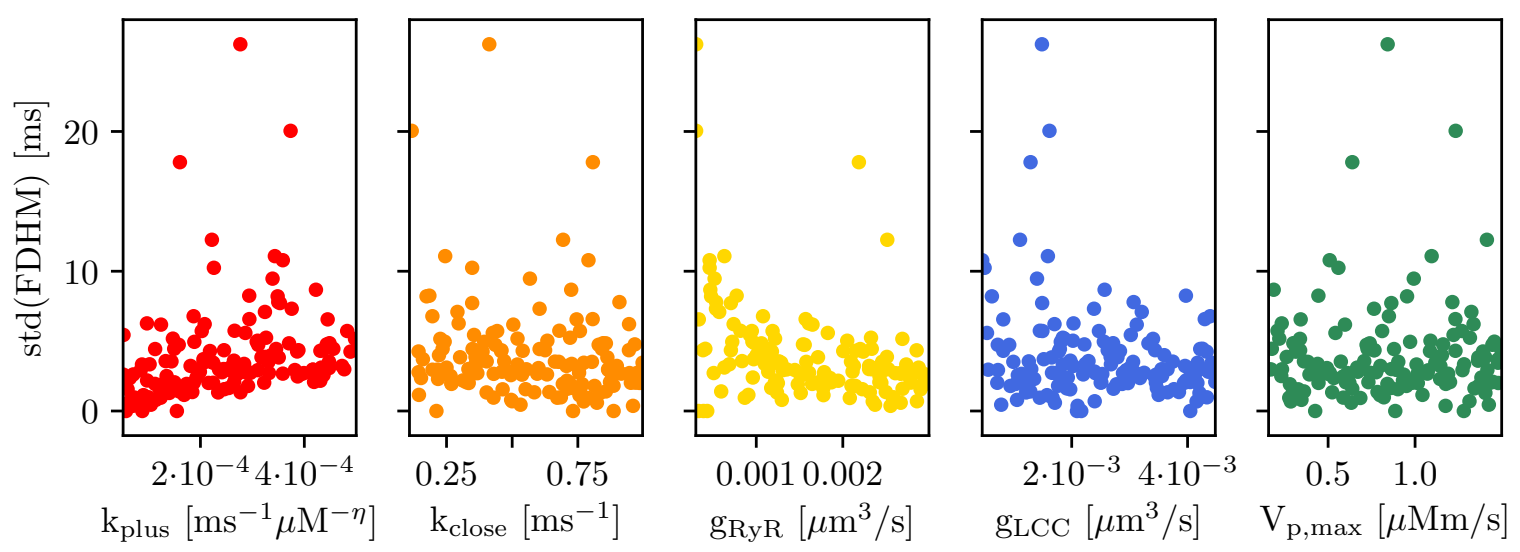

Figure 4.5 - Standard deviation of the FDHM biomarker as function of the varied parameters $k_{\text {plus }}, k_{\text {close }}, g_{\mathrm{RyR}}, g_{\mathrm{LCC}}$ and $V p_{\max }$.

There have been many and ongoing discussions on how sparks and quarks have to be defined $[12,89,92,93]$. We decided to adopt a description of what a spark is based on the events that trigger this phenomenon, i.e. the opening of RyR channels (see the dedicated section in 3.1.1). We defined a single opening RyR in a $20 \mathrm{~ms}$ time lapse to be a quark and any number of additional open RyRs in this time windows would transform the quark to a spark. Based on this definition the calcium transient, i.e. the calcium profile during a spark, was determined for each event. A double check on the number of sparks and the calcium profiles that arise from each cleft during the course of a spark simulation showed an overlap in the time series, i.e. the calcium concentration profile did show spikes at the time points when sparks where recorded. Nevertheless this relation was not checked quantitatively and is based on observations of a handful of simulation runs. The choice of what a spark or quark event is definitively affects the spark biomarkers. This choice is challenging since sparks cannot be quantitatively and rigorously described across different experimental studies yet [5, 13, 49, 113].

Further, the spark simulations are not paced and thus completely in the hands of the system's stochasticity. This lack of control of the system led to strong fluctuations in the biomarkers. In figure 4.5 the standard deviation of the FDHM biomarker shown in figure 3.5 is depicted. The fluctuations are often in the order of the computed biomarker. This effect arises from two major facts: the strong variability in the cleft geometry and the rareness, with which spark events can occur. These fluctuations 
can be observed also in figure 11 of [50], where they are equally strong for both the regular and the Soeller placement. This kind of fluctuations render statements on biomarker behaviour challenging. For example, it is not immediately apparent that with increasing mean occupancy also the mean FDHM of sparks grows. Nevertheless, when trends are observed despite these fluctuations they can for sure be considered to be relevant, as for instance it is the case for the decrease of peak calcium concentration with increasing mean occupancy (see figure 11 in [50]).

The spark simulations in cleftdyn represent the case, in which the cardiomyocyte is left unperturbed for several seconds and the resulting spontaneous events are observed. This setting is clearly not the one that best describes cardiac myocytes in the myocardium. Nevertheless, spark simulations can be descriptive for those microscopic phenomena that occur inside cardiomyocytes that are overruled or concealed by the concerted cellular activity. Phenomena like the spontaneous activation of RyRs cluster, leading to calcium waves $[2,31,38,63,90,91,114]$, which can be hazardous in ventricular cardiomyocytes, can be studied with spark simulations. A deeper understanding of how sparks are generated and how they react to crucial parameter variations or structure modifications can help to find a better definition of what a spark actually represents.

Cleftdyn is a mathematical model that allows in its implementation to close the gap between microscopic subcellular structures and the mesoscopic intracellular calcium and membrane voltage dynamics. Therefore, assessing the nature of quarks and sparks in this context is crucial to deepen the insight on phenomena that originate from them as, e.g., calcium waves. 


\section{Chapter 5}

\section{Discussion and Conclusions}

In the course of this work I studied the impact of different geometrical arrangements of the calcium channels RyR2 in cardiac myocytes on the ion and membrane potential dynamics. Based on recent findings of Jayasinghe, Clowsley and coauthors concerning the geometry of RyR clusters in cardiomyocytes [1], we investigated numerically the influence of RyR placement on excitation contraction coupling. The main result is published in [50] and is discussed ibidem and in section 3.4. I further investigated and tested a method for the creation of surrogate models using training data generated by the complex multiscale model, cleftdyn, I employed here and discuss its features and potential applications in section 4.2 .

\subsection{Impact of Geometry on Global Cardiac My- ocyte Functionality}

For many years mathematical models of cardiac cells assumed the geometrical arrangement of RyRs to be lattice like with densely packed channels [12, 24-28, 35, 45]. Jayasinghe, Clowsley et al. recently showed, using an experimental technique known as DNA-Paint, that the shape of RyR clusters is not lattice like, as previously assumed, but elongated [1]. They also found the RyR channels to be less densely packed than until then believed. Due to this new experimental result the question whether a modification of the RyR cluster would affect the dynamics of the entire cardiomyocyte became strongly relevant. Therefore we addressed this question using the model developed in the Falcke lab of the Max Delbrück Center for Molecular Medicine in Berlin [23, 24, 35, 43-47]. This multiscale model, termed cleftdyn, allows to perform numerical simulations of the processes in cardiomyocytes considering the calcium dynamics from the very small detail of the CRU to mesoscopic ion diffusion in the subcellular space. It thus closes a gap between the calcium diffusion in dyadic clefts and the calcium diffusion in the cytosol. The respective time scales of processes on the different domains are covered as well, making cleftdyn the perfect tool for studying the impact changes of the microscopic structure have on macroscopic phenomena. We studied the impact geometrical changes have on the behaviour of the cell by considering 
variations of five input parameters that control the functions of CRUs on the model's output, which we called biomarkers. Biomarkers, as e.g. action potential duration or peak calcium concentrations, represent the cardiomyocyte functions on a global cell scale.

In the performed study we showed that changes in the arrangement of RyRs in CRUs, i.e. from a regular to a "random walk"-like placement, significantly alter the behaviour of selected biomarkers (detailed in section 3.4.1). This means that the choice of the shape of RyR clusters plays an important role for the output of mathematical models that are used to describe intra-cardiomyocyte calcium dynamics. We further found that not only the shape of the RyR cluster matters, but also the packing of the channels. Employing the concept of occupancy from geometry and varying the gaps between subsequent RyRs we could show that for a given placement the model's output depends on the channel density as described in sections 3.4.2 and 3.4.3.

Some studies already considered deviations from regular and lattice like arrangement of RyRs in CRUs [25, 27]; further investigations did also alter the density of RyR packing in the channel clusters [13]. Nevertheless no study, to my knowledge, considers both aspects of different cluster shape and channel density and is, at the same time, able to simulate the impact they have on a broader cell-wide scale. In contrast, we investigated how the geometrical structure of CRUs affects the cardiomyocyte functionality. For example, the values of $\mathrm{APD}_{90}$ and peak calcium concentration decrease up to $20 \%$ when the mean occupancy of RyR channels is increased by a factor of 0.5. These results call for a critical assessment of existing mathematical models that focus on ECC and spatial ionic dynamics in cardiomyocytes and approximate the channels in the $\mathrm{CRU}$ as to be densely packed $[4,29,30,33,38,40,48,115]$. These models that consider CRU and channel dynamics together with ion diffusion in the whole cell have to be revised concerning approximations made on RyR clusters in order to reliably describe ECC and ion dynamics in cardiomyocytes.

Finally, as I discussed in section 4.2.2, I confirmed the strong variability of calcium sparks $[13,93]$ and I concluded that more experimental insight is needed here to fully understand the characteristics of spontaneous calcium release in cardiac myocytes.

\subsection{Construction of a Surrogate Model}

In recent years more attention has been paid to the application of statistical methods that assist computationally expensive cardiac models $[52,55]$. The need for a surrogate (or meta model) of a mathematical model increases with its complexity. This is related to the improvements complex models make in describing in detail experiments and ultimately nature. A surrogate model is constructed with the output of a complex numerical model and provides a short cut for computationally expensive simulation runs. It can thus be used to sample the input parameter space finer than the original model is able to do (with limited computational resources). An interplay of the two models allows broader investigations of the studied processes. In this regard a meta model can function for a complex numerical model as this does for experimental studies, 
i.e. it makes insight possible where the higher model (and finally the experiment) lack the computational power for a complete investigation. In this thesis I presented one method for the construction of a surrogate model, but many more exist, as I shall outline in the following section 5.3.

The application of statistical methods that allow analysing computationally expensive cardiac models has become more and more relevant in recent years [52, 55]. During the course of my work the input parameters of the mathematical model, cleftdyn, needed to be calibrated after the improvements done on the modelling of the RyR clusters. During this process we employed techniques from statistics $[51,52,54]$ in order to compensate for the small amount of simulation runs that the costly cleftdyn is able to produce. We focused on one specific method, i.e. the polynomial chaos expansion (PCE) $[56,58,106]$. During my work I showed how PCE regression can be used on the output of cleftdyn to construct a meta model called surrogate model, that is able to reproduce the given output without the need of thousands of very expensive runs of the full multiscale model. In sections 3.3.2 and 4.2 I showed how this method can be applied to a system like cleftdyn. In particular the optimisation of the regression fit using cross validation turned out to be crucial for the creation of a reliable surrogate model. In the light of the new meta model I then discussed the data of the publication [50] again in section 4.2.1. I improved the method for the calibration of the input parameters of cleftdyn and demonstrated that the optimised surrogate model confirms the dependencies of the biomarkers on the input parameters found in [50].

Furthermore, the surrogate model shows that the output of cleftdyn can be approximated using polynomial functions with low degrees. For the case of AP simulations third order polynomials are able to describe the relations between selected input parameters and biomarkers, while for spark simulations first order polynomials already suffice. A strongly nonlinear behaviour in the considered parameter-biomarker relations is thus not found.

\subsection{Outlook}

With the established Soeller placement of RyR channels [1] one can focus on studying geometrical modifications that correspond to cardiomyocyte diseases as for example t-tubule disruption $[38,49]$. In cleftdyn this is easily achieved modifying the ratio between the number of RyRs and LCCs in CRUs. Such an investigation would help to understand the emergence of function threatening calcium waves [2, 90, 114, 116-118].

In this study in cleftdyn we employed an electrophysiology model that has its focus on rabbit ventricular cardiomyocytes, i.e. the Mahajan model [37]. Further improvements in cleftdyn were accomplished ${ }^{1}$, in particular the inclusion of the ten Tusscher ionic model for human ventricular cardiomyocytes [36]. It would be desirable to perform a similar study as the one done here with this modification and compare the results.

\footnotetext{
${ }^{1}$ Personal communication with M. Falcke.
} 
Moreover, since the spark simulations are subject to a strong variability in the output, it can be pathbreaking to perform further runs of this simulation type and test further definitions of sparks and quarks as they are outlined in [93]. This would refine the definition of a spark and show how sparks could be systematically studied in experimental investigations. Further, also events that involve more than one single CRU, so-called macro sparks, could be studied as well. Together with further spark simulations it would be desirable to assess, with enlarged domains and longer simulation periods, whether long-lasting sparks or calcium waves arise when the RyRs are altered in their function accordingly $[2,5]$.

With respect to the creation of a surrogate model further approaches could be tested. The simplest modifications would include different regression methods for the PCE as for example a so-called Ridge regression. Furthermore, methods as, e.g. the principal component analysis [119] could be used to reduce the dimensionality of the data. The $k$-dimensional tree [120] could then be employed on the dimensional reduced data set to generate a meta model.

Since cleftdyn has a high number of possible input parameters, it is also desirable to use methods that are able to cope with high dimensional spaces. For instance a method known as low-rank tensor approximation [56, 121, 122] could be used to generate a meta model in the case the number of the varied input parameters increases from the one used here.

\subsection{Conclusion}

Based on recent experimental findings on the shape of RyR clusters, I addressed the question whether different arrangements of calcium channels in CRUs affect the dynamics of cardiomyocytes. The question stems from the crucial role CRUs play for the contraction of heart muscle cells.

I found that the type of geometrical arrangement of RyRs significantly affects the global calcium and AP dynamics of the cell. Not only the classically employed regular, lattice-like arrangement influences the cell's biomarkers differently than the new Soeller placement, but also within the latter configuration variations exist. These are linked to the occupancy of the RyR channels in the dyadic cleft.

Finally, in the process of output analysis I tested and applied PCE regression as one method for the creation of reliable surrogate models. Such a model can be used as a tool for parameter calibration in complex models like cleftdyn or as a computationally efficient predictor approach for exploring the parameter space of the (expensive) complex model.

This study confirmed the necessity to consider microscopic subcellular structures in the modelling of cardiomyocytes, since they have a significant impact on the cell's functionality, which is vital for its contraction and, ultimately, for a properly beating heart. 


\section{References}

[1] I. Jayasinghe, A. H. Clowsley, R. Lin, T. Lutz, C. Harrison, E. Green, D. Baddeley, L. Di Michele, and C. Soeller. "True Molecular Scale Visualization of Variable Clustering Properties of Ryanodine Receptors". en. In: Cell Reports 22.2 (Jan. 2018), pp. 557-567. DOI: 10.1016/j.celrep.2017.12.045.

[2] J. A. Wasserstrom et al. "Variability in Timing of Spontaneous Calcium Release in the Intact Rat Heart Is Determined by the Time Course of Sarcoplasmic Reticulum Calcium Load". en. In: Circulation Research 107.9 (Oct. 2010), pp. 1117-1126. DOI: 10.1161/CIRCRESAHA.110.229294.

[3] L.-S. Song, E. A. Sobie, S. McCulle, W. J. Lederer, C. W. Balke, and H. Cheng. "Orphaned ryanodine receptors in the failing heart". en. In: Proceedings of the National Academy of Sciences 103.11 (Mar. 2006), pp. 4305-4310. DOI: 10.1073/pnas.0509324103.

[4] M. Nivala, Z. Song, J. N. Weiss, and Z. Qu. "T-tubule disruption promotes calcium alternans in failing ventricular myocytes: Mechanistic insights from computational modeling". en. In: Journal of Molecular and Cellular Cardiology 79 (Feb. 2015), pp. 32-41. DOI: 10.1016/j.yjmcc.2014.10.018.

[5] A. V. Zima, E. Picht, D. M. Bers, and L. A. Blatter. "Partial Inhibition of Sarcoplasmic Reticulum Ca Release Evokes Long-Lasting Ca Release Events in Ventricular Myocytes: Role of Luminal Ca in Termination of Ca Release". en. In: Biophysical Journal 94.5 (Mar. 2008), pp. 1867-1879. DOI: 10.1529/biophysj. 107.114694.

[6] M. M. Maleckar, A. G. Edwards, W. E. Louch, and G. T. Lines. "Studying dyadic structure-function relationships: a review of current modeling approaches and new insights into Ca2+ (mis)handling:" en. In: Clinical Medicine Insights: Cardiology (Apr. 2017). DOI: 10.1177/1179546817698602.

[7] X. Shen et al. "3D dSTORM imaging reveals novel detail of ryanodine receptor localization in rat cardiac myocytes". en. In: The Journal of Physiology 597.2 (Jan. 2019), pp. 399-418. DOI: 10.1113/JP277360.

[8] E. Wagner et al. "Stimulated Emission Depletion Live-Cell Super-Resolution Imaging Shows Proliferative Remodeling of T-Tubule Membrane Structures After Myocardial Infarction". en. In: Circulation Research 111.4 (Aug. 2012), pp. 402-414. DOI: 10.1161/CIRCRESAHA.112.274530.

[9] E. A. Rog-Zielinska, C. M. Johnston, E. T. O'Toole, M. Morphew, A. Hoenger, and P. Kohl. "Electron tomography of rabbit cardiomyocyte three-dimensional ultrastructure". en. In: Progress in Biophysics and Molecular Biology 121.2 (July 2016), pp. 77-84. DOI: 10.1016/j.pbiomolbio.2016.05.005. 
[10] R. A. Burton et al. "Caveolae in Rabbit Ventricular Myocytes: Distribution and Dynamic Diminution after Cell Isolation". en. In: Biophysical Journal 113.5 (Sept. 2017), pp. 1047-1059. DOI: 10.1016/j.bpj.2017.07.026.

[11] M. S. Jafri. "Models of Excitation-Contraction Coupling in Cardiac Ventricular Myocytes". en. In: Bioinformatics and Drug Discovery. Ed. by R. S. Larson. Vol. 910. Totowa, NJ: Humana Press, 2012, pp. 309-335. Dor: 10.1007/978-161779-965-5_14.

[12] M. A. Walker, G. S. Williams, T. Kohl, S. E. Lehnart, M. S. Jafri, J. L. Greenstein, W. Lederer, and R. L. Winslow. "Superresolution Modeling of Calcium Release in the Heart". en. In: Biophysical Journal 107.12 (Dec. 2014), pp. 3018-3029. DOI: 10.1016/j.bpj.2014.11.003.

[13] W. E. Louch, J. Hake, H. K. Mørk, K. Hougen, B. Skrbic, D. Ursu, T. Tønnessen, I. Sjaastad, and O. M. Sejersted. "Slow Ca2+ sparks de-synchronize Ca2+ release in failing cardiomyocytes: Evidence for altered configuration of Ca2+ release units?" en. In: Journal of Molecular and Cellular Cardiology 58 (May 2013), pp. 41-52. DOI: 10.1016/j.yjmcc.2013.01.014.

[14] J. A. Negroni and E. C. Lascano. "Simulation of steady state and transient cardiac muscle response experiments with a Huxley-based contraction model". en. In: Journal of Molecular and Cellular Cardiology 45.2 (Aug. 2008), pp. 300312. DOI: 10.1016/j.yjmcc.2008.04.012.

[15] D. Baddeley, I. D. Jayasinghe, L. Lam, S. Rossberger, M. B. Cannell, and C. Soeller. "Optical single-channel resolution imaging of the ryanodine receptor distribution in rat cardiac myocytes". en. In: Proceedings of the National Academy of Sciences 106.52 (Dec. 2009), pp. 22275-22280. DOI: 10.1073/pnas.0908971106.

[16] I. D. Jayasinghe, M. B. Cannell, and C. Soeller. "Organization of Ryanodine Receptors, Transverse Tubules, and Sodium-Calcium Exchanger in Rat Myocytes". en. In: Biophysical Journal 97.10 (Nov. 2009), pp. 2664-2673. DOI: 10.1016/j.bpj.2009.08.036.

[17] S. Brandenburg et al. "Axial tubule junctions control rapid calcium signaling in atria". en. In: Journal of Clinical Investigation 126.10 (Sept. 2016), pp. 39994015. DOI: $10.1172 / \mathrm{JCI} 88241$.

[18] C. Ounkomol, S. Seshamani, M. M. Maleckar, F. Collman, and G. R. Johnson. "Label-free prediction of three-dimensional fluorescence images from transmittedlight microscopy". en. In: Nature Methods 15.11 (Nov. 2018), pp. 917-920. DOI: 10.1038/s41592-018-0111-2.

[19] A. V. Zima, E. Picht, D. M. Bers, and L. A. Blatter. "Termination of Cardiac Ca2+ Sparks: Role of Intra-SR [Ca2+], Release Flux, and Intra-SR Ca2+ Diffusion". en. In: Circulation Research 103.8 (Oct. 2008), e105-e115. DOI: 10.1161/CIRCRESAHA.107.183236.

[20] L. A. Blatter, J. Kockskämper, K. A. Sheehan, A. V. Zima, J. Hüser, and S. L. Lipsius. "Local calcium gradients during excitation-contraction coupling and alternans in atrial myocytes". en. In: The Journal of Physiology 546.1 (Jan. 2003), pp. 19-31. DOI: 10.1113/jphysiol.2002.025239. 
[21] Q. Lou, A. E. Belevych, P. B. Radwański, B. Liu, A. Kalyanasundaram, B. C. Knollmann, V. V. Fedorov, and S. Györke. "Alternating membrane potential/calcium interplay underlies repetitive focal activity in a genetic model of calcium-dependent atrial arrhythmias: Alternating $V$ m $/ \mathrm{Ca}$ interplay underlies repetitive focal activity". en. In: The Journal of Physiology 593.6 (Mar. 2015), pp. 1443-1458. DOI: 10.1113/jphysiol.2014.280784.

[22] R. Jungmann, M. S. Avendaño, J. B. Woehrstein, M. Dai, W. M. Shih, and P. Yin. "Multiplexed 3D cellular super-resolution imaging with DNA-PAINT and Exchange-PAINT". en. In: Nature Methods 11.3 (Mar. 2014), pp. 313-318. DOI: $10.1038 /$ nmeth.2835.

[23] S. Rüdiger, J. Shuai, W. Huisinga, C. Nagaiah, G. Warnecke, I. Parker, and M. Falcke. "Hybrid Stochastic and Deterministic Simulations of Calcium Blips". en. In: Biophysical Journal 93.6 (Sept. 2007), pp. 1847-1857. DOI: 10.1529/ biophysj.106.099879.

[24] T. Schendel and M. Falcke. "Efficient and Detailed Model of the Local Ca2+ Release Unit in the Ventricular Cardiac Myocyte". In: Genome informatics. International Conference on Genome Informatics 22 (Jan. 2010), pp. 142-55. DOI: 10.1142/9781848165786_0012.

[25] M. A. Walker, T. Kohl, S. E. Lehnart, J. L. Greenstein, W. J. Lederer, and R. L. Winslow. "On the Adjacency Matrix of RyR2 Cluster Structures". en. In: PLOS Computational Biology 11.11 (Nov. 2015). Ed. by J. J. Saucerman, e1004521. DOI: 10.1371/journal.pcbi.1004521.

[26] M. B. Cannell and C. Soeller. "Numerical analysis of ryanodine receptor activation by L-type channel activity in the cardiac muscle diad." In: Biophysical Journal 73.1 (July 1997), pp. 112-122. DOI: 10.1016/S0006-3495(97)78052-4.

[27] M. Cannell, C. Kong, M. Imtiaz, and D. Laver. "Control of Sarcoplasmic Reticulum Ca2+ Release by Stochastic RyR Gating within a 3D Model of the Cardiac Dyad and Importance of Induction Decay for CICR Termination". en. In: Biophysical Journal 104.10 (May 2013), pp. 2149-2159. DOI: 10.1016/j.bpj. 2013.03.058.

[28] J. Hake, A. G. Edwards, Z. Yu, P. M. Kekenes-Huskey, A. P. Michailova, J. A. McCammon, M. J. Holst, M. Hoshijima, and A. D. McCulloch. "Modelling cardiac calcium sparks in a three-dimensional reconstruction of a calcium release unit: Calcium sparks in reconstructed release unit". en. In: The Journal of Physiology 590.18 (Sept. 2012), pp. 4403-4422. DOI: 10.1113/jphysiol.2012. 227926.

[29] J. G. Restrepo, J. N. Weiss, and A. Karma. "Calsequestrin-Mediated Mechanism for Cellular Calcium Transient Alternans". en. In: Biophysical Journal 95.8 (Oct. 2008), pp. 3767-3789. DOI: 10.1529/biophysj.108.130419.

[30] A. Hatano, J.-i. Okada, T. Washio, T. Hisada, and S. Sugiura. "A ThreeDimensional Simulation Model of Cardiomyocyte Integrating ExcitationContraction Coupling and Metabolism". en. In: Biophysical Journal 101.11 (Dec. 2011), pp. 2601-2610. DOI: 10.1016/j.bpj.2011.10.020. 
[31] P. Li, W. Wei, X. Cai, C. Soeller, M. B. Cannell, and A. V. Holden. "Computational modelling of the initiation and development of spontaneous intracellular Ca2+ waves in ventricular myocytes". en. In: Philosophical Transactions of the Royal Society A: Mathematical, Physical and Engineering Sciences 368.1925 (Aug. 2010), pp. 3953-3965. DOI: 10.1098/rsta.2010.0146.

[32] R. Rovetti, X. Cui, A. Garfinkel, J. N. Weiss, and Z. Qu. "Spark-Induced Sparks As a Mechanism of Intracellular Calcium Alternans in Cardiac Myocytes". en. In: Circulation Research 106.10 (May 2010), pp. 1582-1591. DOI: 10.1161/ CIRCRESAHA.109.213975.

[33] M. Nivala, E. de Lange, R. Rovetti, and Z. Qu. "Computational Modeling and Numerical Methods for Spatiotemporal Calcium Cycling in Ventricular Myocytes". In: Frontiers in Physiology 3 (2012). DOI: 10.3389/fphys.2012.00114.

[34] M. A. Walker, V. Gurev, J. J. Rice, J. L. Greenstein, and R. L. Winslow. "Estimating the probabilities of rare arrhythmic events in multiscale computational models of cardiac cells and tissue". en. In: PLOS Computational Biology 13.11 (Nov. 2017), e1005783. DOI: 10.1371/journal.pcbi.1005783.

[35] J. Vierheller, W. Neubert, M. Falcke, S. H. Gilbert, and N. Chamakuri. "A multiscale computational model of spatially resolved calcium cycling in cardiac myocytes: from detailed cleft dynamics to the whole cell concentration profiles". In: Frontiers in Physiology 6 (Sept. 2015). DOI: 10.3389/fphys.2015.00255.

[36] K. H. W. J. ten Tusscher, D. Noble, P. J. Noble, and A. V. Panfilov. "A model for human ventricular tissue". en. In: American Journal of Physiology-Heart and Circulatory Physiology 286.4 (Apr. 2004), H1573-H1589. DOI: 10.1152/ajpheart. 00794.2003.

[37] A. Mahajan et al. "A Rabbit Ventricular Action Potential Model Replicating Cardiac Dynamics at Rapid Heart Rates". In: Biophysical Journal 94.2 (Jan. 2008), pp. 392-410. DOI: 10.1529/biophysj.106.98160.

[38] J. K. Singh et al. "T-tubule remodeling and increased heterogeneity of calcium release during the progression to heart failure in intact rat ventricle". en. In: Physiological Reports 5.24 (Dec. 2017), e13540. DOI: 10.14814/phy2.13540.

[39] Z. Qu, M. B. Liu, and M. Nivala. "A unified theory of calcium alternans in ventricular myocytes". en. In: Scientific Reports 6.1 (Dec. 2016). DoI: 10.1038/ srep35625.

[40] Y. Shiferaw. "Nonlinear onset of calcium wave propagation in cardiac cells". en. In: Physical Review E 94.3 (Sept. 2016). DOI: 10.1103/PhysRevE.94.032405.

[41] A. Bueno-Orovio, E. M. Cherry, and F. H. Fenton. "Minimal model for human ventricular action potentials in tissue". en. In: Journal of Theoretical Biology 253.3 (Aug. 2008), pp. 544-560. DOI: 10.1016/j.jtbi.2008.03.029.

[42] Luo C H and Rudy Y. "A dynamic model of the cardiac ventricular action potential. I. Simulations of ionic currents and concentration changes." In: Circulation Research 74.6 (June 1994), pp. 1071-1096. DOI: 10.1161/01.RES.74.6.1071. 
[43] N. Chamakuri, W. Neubert, S. Gilbert, J. Vierheller, G. Warnecke, and M. Falcke. "Multiscale Modeling and Numerical Simulation of Calcium Cycling in Cardiac Myocytes". en. In: Multiscale Modeling 65 Simulation 16.3 (Jan. 2018), pp. 1115-1145. DOI: 10.1137/17M1121639.

[44] C. Nagaiah, S. Rüdiger, G. Warnecke, and M. Falcke. "Adaptive space and time numerical simulation of reaction-diffusion models for intracellular calcium dynamics". en. In: Applied Mathematics and Computation 218.20 (June 2012), pp. 10194-10210. DOI: 10.1016/j.amc.2012.03.100.

[45] T. Schendel, R. Thul, J. Sneyd, and M. Falcke. "How does the ryanodine receptor in the ventricular myocyte wake up: by a single or by multiple open L-type Ca2+ channels?" en. In: European Biophysics Journal 41.1 (Jan. 2012), pp. 27-39. DOI: $10.1007 / \mathrm{s} 00249-011-0755-7$.

[46] C. Nagaiah, S. Rüdiger, G. Warnecke, and M. Falcke. "Adaptive numerical simulation of intracellular calcium dynamics using domain decomposition methods". en. In: Applied Numerical Mathematics 58.11 (Nov. 2008), pp. 1658-1674. DOI: 10.1016/j.apnum.2007.10.003.

[47] J. Vierheller. "Modelling excitation coupling in ventricular cardiac myocytes". PhD thesis. Berlin: Max-Delbrück-Centrum für Molekulare Medizin, May 2018.

[48] J. G. Restrepo and A. Karma. "Spatiotemporal intracellular calcium dynamics during cardiac alternans". en. In: Chaos: An Interdisciplinary Journal of Nonlinear Science 19.3 (Sept. 2009), p. 037115. DOI: 10.1063/1.3207835.

[49] A. R. Lyon, K. T. MacLeod, Y. Zhang, E. Garcia, G. K. Kanda, M. J. Lab, Y. E. Korchev, S. E. Harding, and J. Gorelik. "Loss of T-tubules and other changes to surface topography in ventricular myocytes from failing human and rat heart". en. In: Proceedings of the National Academy of Sciences 106.16 (Apr. 2009), pp. 6854-6859. DOI: 10.1073/pnas.0809777106.

[50] F. G. Cosi, W. Giese, W. Neubert, S. Luther, N. Chamakuri, U. Parlitz, and M. Falcke. "Multiscale Modeling of Dyadic Structure-Function Relation in Ventricular Cardiac Myocytes". en. In: Biophysical Journal (Sept. 2019). DoI: 10.1016/j.bpj.2019.09.023.

[51] G. R. Mirams, P. Pathmanathan, R. A. Gray, P. Challenor, and R. H. Clayton. "Uncertainty and variability in computational and mathematical models of cardiac physiology: Uncertainty and variability in cardiac models". en. In: The Journal of Physiology 594.23 (Dec. 2016), pp. 6833-6847. DOI: 10.1113/JP271671.

[52] E. T. Y. Chang, M. Strong, and R. H. Clayton. "Bayesian Sensitivity Analysis of a Cardiac Cell Model Using a Gaussian Process Emulator". en. In: PLoS ONE 10.6 (June 2015). Ed. by K. Burrage, e0130252. DOI: 10.1371/journal. pone.0130252.

[53] R. H. Johnstone, E. T. Chang, R. Bardenet, T. P. de Boer, D. J. Gavaghan, P. Pathmanathan, R. H. Clayton, and G. R. Mirams. "Uncertainty and variability in models of the cardiac action potential: Can we build trustworthy models?" en. In: Journal of Molecular and Cellular Cardiology 96 (July 2016), pp. 49-62. DOI: $10.1016 /$ j.yjmcc.2015.11.018. 
[54] O. J. Britton, A. Bueno-Orovio, K. Van Ammel, H. R. Lu, R. Towart, D. J. Gallacher, and B. Rodriguez. "Experimentally calibrated population of models predicts and explains intersubject variability in cardiac cellular electrophysiology". en. In: Proceedings of the National Academy of Sciences 110.23 (June 2013), E2098-E2105. DOI: 10.1073/pnas.1304382110.

[55] F. Sahli Costabal, J. Yao, and E. Kuhl. "Predicting the cardiac toxicity of drugs using a novel multiscale exposure-response simulator". en. In: Computer Methods in Biomechanics and Biomedical Engineering 21.3 (Feb. 2018), pp. 232246. DOI: $10.1080 / 10255842.2018 .1439479$.

[56] B. Sudret, S. Marelli, and J. Wiart. "Surrogate models for uncertainty quantification: An overview". en. In: 2017 11th European Conference on Antennas and Propagation (EUCAP). Paris, France: IEEE, Mar. 2017, pp. 793-797. DOI: 10.23919/EuCAP.2017.7928679.

[57] M. C. Kennedy and A. O'Hagan. "Predicting the output from a complex computer code when fast approximations are available". en. In: Biometrika 87.1 (Mar. 2000), pp. 1-13. DOI: 10.1093/biomet/87.1.1.

[58] D. Xiu and G. E. Karniadakis. "The Wiener-Askey Polynomial Chaos for Stochastic Differential Equations". en. In: SIAM Journal on Scientific Computing 24.2 (Jan. 2002), pp. 619-644. DOI: 10.1137/S1064827501387826.

[59] S. Galice, Y. Xie, Y. Yang, D. Sato, and D. M. Bers. "Size Matters: Ryanodine Receptor Cluster Size Affects Arrhythmogenic Sarcoplasmic Reticulum Calcium Release". en. In: Journal of the American Heart Association 7.13 (July 2018). DOI: 10.1161/JAHA.118.008724.

[60] "Medical gallery of Blausen Medical 2014". en. In: WikiJournal of Medicine 1.2 (2014). DOI: $10.15347 / \mathrm{wjm} / 2014.010$.

[61] J. A. Negroni and E. C. Lascano. "A Cardiac Muscle Model Relating Sarcomere Dynamics to Calcium Kinetics". en. In: Journal of Molecular and Cellular Cardiology 28.5 (May 1996), pp. 915-929. DOI: 10.1006/jmcc.1996.0086.

[62] J. J. Rice, F. Wang, D. M. Bers, and P. P. de Tombe. "Approximate Model of Cooperative Activation and Crossbridge Cycling in Cardiac Muscle Using Ordinary Differential Equations". en. In: Biophysical Journal 95.5 (Sept. 2008), pp. 2368-2390. DOI: 10.1529/biophysj.107.119487.

[63] J.-i. Okada, S. Sugiura, S. Nishimura, and T. Hisada. "Three-dimensional simulation of calcium waves and contraction in cardiomyocytes using the finite element method". en. In: American Journal of Physiology-Cell Physiology 288.3 (Mar. 2005), pp. C510-C522. DOI: 10.1152/ajpcell.00261.2004.

[64] C. Pinali, H. Bennett, J. B. Davenport, A. W. Trafford, and A. Kitmitto. "Three-dimensional reconstruction of cardiac sarcoplasmic reticulum reveals a continuous network linking transverse-tubules: this organization is perturbed in heart failure". eng. In: Circulation Research 113.11 (Nov. 2013), pp. 1219-1230. DOI: 10.1161/CIRCRESAHA.113.301348.

[65] D. M. Bers. "Cardiac excitation-contraction coupling". en. In: Nature 415.6868 (Jan. 2002), pp. 198-205. DOI: 10.1038/415198a. 
[66] D. M. Bers. "Calcium Cycling and Signaling in Cardiac Myocytes". en. In: Annual Review of Physiology 70.1 (Mar. 2008), pp. 23-49. DOI: 10.1146/annurev. physiol.70.113006.100455.

[67] R. L. Winslow, M. A. Walker, and J. L. Greenstein. "Modeling calcium regulation of contraction, energetics, signaling, and transcription in the cardiac myocyte: Modeling of cellular calcium dynamics". en. In: Wiley Interdisciplinary Reviews: Systems Biology and Medicine 8.1 (Jan. 2016), pp. 37-67. DOI: 10.1002/wsbm. 1322.

[68] Shannon Thomas R., Pogwizd Steven M., and Bers Donald M. "Elevated Sarcoplasmic Reticulum Ca2+ Leak in Intact Ventricular Myocytes From Rabbits in Heart Failure". In: Circulation Research 93.7 (Oct. 2003). Publisher: American Heart Association, pp. 592-594. DOI: 10.1161/01.RES.0000093399.11734.B3.

[69] Weber Christopher R., Ginsburg Kenneth S., and Bers Donald M. "Cardiac Submembrane $[\mathrm{Na}+]$ Transients Sensed by Na+-Ca2+ Exchange Current". In: Circulation Research 92.9 (May 2003), pp. 950-952. DOI: 10.1161/01.RES. $0000071747.61468 .7 \mathrm{~F}$.

[70] C. Franzini-Armstrong, F. Protasi, and V. Ramesh. "Shape, Size, and Distribution of Ca2+ Release Units and Couplons in Skeletal and Cardiac Muscles". en. In: Biophysical Journal 77.3 (Sept. 1999), pp. 1528-1539. DOI: 10.1016/S00063495(99)77000-1.

[71] C. Franzini-Armstrong, F. Protasi, and P. Tijskens. "The Assembly of Calcium Release Units in Cardiac Muscle". en. In: Annals of the New York Academy of Sciences 1047.1 (2005), pp. 76-85. DOI: 10.1196/annals.1341.007.

[72] C. Soeller and M. B. Cannell. "Numerical simulation of local calcium movements during L-type calcium channel gating in the cardiac diad". en. In: Biophysical Journal 73.1 (July 1997), pp. 97-111. DOI: 10.1016/S0006-3495(97)78051-2.

[73] M. Radermacher, V. Rao, R. Grassucci, J. Frank, A. P. Timerman, S. Fleischer, and T. Wagenknecht. "Cryo-electron microscopy and three-dimensional reconstruction of the calcium release channel/ryanodine receptor from skeletal muscle." en. In: Journal of Cell Biology 127.2 (Oct. 1994). Publisher: The Rockefeller University Press, pp. 411-423. DOI: 10.1083/jcb.127.2.411.

[74] D. M. Bers and V. M. Stiffel. "Ratio of ryanodine to dihydropyridine receptors in cardiac and skeletal muscle and implications for E-C coupling". en. In: American Journal of Physiology-Cell Physiology 264.6 (June 1993), pp. C1587-C1593. DOI: 10.1152/ajpcell.1993.264.6.C1587.

[75] M. Heilemann, S. van de Linde, M. Schüttpelz, R. Kasper, B. Seefeldt, A. Mukherjee, P. Tinnefeld, and M. Sauer. "Subdiffraction-Resolution Fluorescence Imaging with Conventional Fluorescent Probes". en. In: Angewandte Chemie International Edition 47.33 (Aug. 2008), pp. 6172-6176. DOI: 10.1002/anie. 200802376.

[76] Y. Chen-Izu, S. L. McCulle, C. W. Ward, C. Soeller, B. M. Allen, C. Rabang, M. B. Cannell, C. W. Balke, and L. T. Izu. "Three-Dimensional Distribution of Ryanodine Receptor Clusters in Cardiac Myocytes". en. In: Biophysical Journal 91.1 (July 2006), pp. 1-13. DOI: 10.1529/biophysj.105.077180. 
[77] A. Takahashi, P. Camacho, J. D. Lechleiter, and B. Herman. "Measurement of Intracellular Calcium". en. In: Physiological Reviews 79.4 (Jan. 1999), pp. 10891125. DOI: 10.1152/physrev.1999.79.4.1089.

[78] M. D. Stern, L.-S. Song, H. Cheng, J. S. Sham, H. T. Yang, K. R. Boheler, and E. Ríos. "Local Control Models of Cardiac Excitation-Contraction Coupling: A Possible Role for Allosteric Interactions between Ryanodine Receptors". en. In: The Journal of General Physiology 113.3 (Mar. 1999), pp. 469-489. DOI: 10.1085/jgp.113.3.469.

[79] M. S. Jafri, J. J. Rice, and R. L. Winslow. "Cardiac Ca2+ Dynamics: The Roles of Ryanodine Receptor Adaptation and Sarcoplasmic Reticulum Load". en. In: Biophysical Journal 74.3 (Mar. 1998), pp. 1149-1168. DOI: 10.1016/S00063495(98)77832-4.

[80] R. Hinch, J. Greenstein, A. Tanskanen, L. Xu, and R. Winslow. "A Simplified Local Control Model of Calcium-Induced Calcium Release in Cardiac Ventricular Myocytes". en. In: Biophysical Journal 87.6 (Dec. 2004), pp. 3723-3736. DOI: 10.1529/biophysj.104.049973.

[81] D. T. Gillespie. "A general method for numerically simulating the stochastic time evolution of coupled chemical reactions". en. In: Journal of Computational Physics 22.4 (Dec. 1976), pp. 403-434. DOI: 10.1016/0021-9991(76)90041-3.

[82] D. T. Gillespie. "Exact stochastic simulation of coupled chemical reactions". en. In: The Journal of Physical Chemistry 81.25 (Dec. 1977), pp. 2340-2361. DOI: 10.1021/j100540a008.

[83] D. T. Gillespie. "Stochastic Simulation of Chemical Kinetics". en. In: Annual Review of Physical Chemistry 58.1 (May 2007), pp. 35-55. DOI: 10.1146/annurev. physchem.58.032806.104637.

[84] P. Bastian, M. Blatt, A. Dedner, C. Engwer, R. Klöfkorn, R. Kornhuber, M. Ohlberger, and O. Sander. "A generic grid interface for parallel and adaptive scientific computing. Part II: implementation and tests in DUNE". en. In: Computing 82.2-3 (July 2008), pp. 121-138. DOI: 10.1007/s00607-008-0004-9.

[85] P. Bastian, F. Heimann, and S. Marnach. "Generic implementation of finite element methods in the Distributed and Unified Numerics Environment (DUNE)". In: Kybernetika 2 (Jan. 2010).

[86] P. Bastian et al. "The DUNE Framework: Basic Concepts and Recent Developments". en. In: arXiv:1909.13672 [cs] (Sept. 2019). arXiv: 1909.13672.

[87] M. P. Forum. MPI: A Message-Passing Interface Standard. Tech. rep. Knoxville, TN, USA, 1994.

[88] P. Gemmell, K. Burrage, B. Rodríguez, and T. A. Quinn. "Rabbit-specific computational modelling of ventricular cell electrophysiology: Using populations of models to explore variability in the response to ischemia". en. In: Progress in Biophysics and Molecular Biology 121.2 (July 2016), pp. 169-184. DOI: 10.1016/j.pbiomolbio.2016.06.003.

[89] H. Cheng, W. Lederer, and M. Cannell. "Calcium sparks: elementary events underlying excitation-contraction coupling in heart muscle". en. In: Science 262.5134 (Oct. 1993), pp. 740-744. DOI: 10.1126/science.8235594. 
[90] L. T. Izu, W. G. Wier, and C. W. Balke. "Evolution of Cardiac Calcium Waves from Stochastic Calcium Sparks". en. In: Biophysical Journal 80.1 (Jan. 2001), pp. 103-120. DOI: 10.1016/S0006-3495(01)75998-X.

[91] M. Nivala, C. Y. Ko, M. Nivala, J. N. Weiss, and Z. Qu. "The emergence of subcellular pacemaker sites for calcium waves and oscillations: Calcium waves and oscillations". en. In: The Journal of Physiology 591.21 (Nov. 2013), pp. 53055320. DOI: $10.1113 /$ jphysiol.2013.259960.

[92] G. S. Williams, A. C. Chikando, H.-T. M. Tuan, E. A. Sobie, W. Lederer, and M. S. Jafri. "Dynamics of Calcium Sparks and Calcium Leak in the Heart". en. In: Biophysical Journal 101.6 (Sept. 2011), pp. 1287-1296. DOI: 10.1016/j.bpj. 2011.07.021.

[93] H. Cheng and W. J. Lederer. "Calcium Sparks". In: Physiological Reviews 88.4 (Oct. 2008), pp. 1491-1545. DOI: 10.1152/physrev.00030.2007.

[94] P. Gemmell, K. Burrage, B. Rodriguez, and T. A. Quinn. "Population of Computational Rabbit-Specific Ventricular Action Potential Models for Investigating Sources of Variability in Cellular Repolarisation". en. In: PLoS ONE 9.2 (Feb. 2014). Ed. by V. E. Bondarenko, e90112. DOI: 10.1371/journal.pone.0090112.

[95] M. D. McKay, R. J. Beckman, and W. J. Conover. "A Comparison of Three Methods for Selecting Values of Input Variables in the Analysis of Output from a Computer Code". In: Technometrics 21.2 (1979), pp. 239-245. DOI: 10.1080/00401706.1979.10489755.

[96] G. E. P. Box. "Sampling and Bayes' Inference in Scientific Modelling and Robustness". en. In: Journal of the Royal Statistical Society. Series A (General) 143.4 (1980), p. 383. DOI: 10.2307/2982063.

[97] D. Tran, A. Kucukelbir, A. B. Dieng, M. Rudolph, D. Liang, and D. M. Blei. "Edward: A library for probabilistic modeling, inference, and criticism". en. In: arXiv:1610.09787 [cs, stat] (Jan. 2017). arXiv: 1610.09787.

[98] M. Abadi et al. "TensorFlow: A System for Large-Scale Machine Learning". In: (Nov. 2016), pp. 265-283.

[99] F. Pedregosa et al. "Scikit-learn: Machine Learning in Python". In: Journal of Machine Learning Research 12 (2011), pp. 2825-2830.

[100] I. Sobol. "Sensitivity Estimates for Nonlinear Mathematical Models". en. In: Matem. Mod. 2 (1990), pp. 112-118.

[101] I. Sobol. "Global sensitivity indices for nonlinear mathematical models and their Monte Carlo estimates". en. In: Mathematics and Computers in Simulation 55.1-3 (Feb. 2001), pp. 271-280. DOI: 10.1016/S0378-4754(00)00270-6.

[102] A. Saltelli and I. M. Sobol'. "Sensitivity analysis for nonlinear mathematical models: numerical experience". In: Matematicheskoe Modelirovanie 7.11 (1995), pp. 16-28.

[103] D. Fedida and W. R. Giles. "Regional variations in action potentials and transient outward current in myocytes isolated from rabbit left ventricle." en. In: The Journal of Physiology 442.1 (Oct. 1991), pp. 191-209. DOI: 10.1113/ jphysiol.1991.sp018789. 
[104] D. A. Golod, R. Kumar, and R. W. Joyner. "Determinants of action potential initiation in isolated rabbit atrial and ventricular myocytes". en. In: American Journal of Physiology-Heart and Circulatory Physiology 274.6 (June 1998), H1902-H1913. DOI: 10.1152/ajpheart.1998.274.6.H1902.

[105] C. Soize and R. Ghanem. "Physical Systems with Random Uncertainties: Chaos Representations with Arbitrary Probability Measure". en. In: SIAM Journal on Scientific Computing 26.2 (Jan. 2004), pp. 395-410. DOI: 10.1137 / S1064827503424505.

[106] G. Blatman and B. Sudret. "Efficient computation of global sensitivity indices using sparse polynomial chaos expansions". en. In: Reliability Engineering 86 System Safety 95.11 (Nov. 2010), pp. 1216-1229. DOI: 10.1016/j.ress.2010.06.015.

[107] B. Sudret. "Global sensitivity analysis using polynomial chaos expansions". en. In: Reliability Engineering \& System Safety 93.7 (July 2008), pp. 964-979. DOI: 10.1016/j.ress.2007.04.002.

[108] R. Tibshirani. "Regression Shrinkage and Selection Via the Lasso". en. In: Journal of the Royal Statistical Society: Series B (Methodological) 58.1 (Jan. 1996), pp. 267-288. DOI: 10.1111/j.2517-6161.1996.tb02080.x.

[109] J. Feinberg and H. P. Langtangen. "Chaospy: An open source tool for designing methods of uncertainty quantification". en. In: Journal of Computational Science 11 (Nov. 2015), pp. 46-57. DOI: 10.1016/j.jocs.2015.08.008.

[110] M. Berveiller, B. Sudret, and M. Lemaire. "Stochastic finite element: a non intrusive approach by regression". en. In: European Journal of Computational Mechanics 15.1-3 (Jan. 2006), pp. 81-92. DOI: 10.3166/remn.15.81-92.

[111] J. Friedman, T. Hastie, and R. Tibshirani. "Regularization Paths for Generalized Linear Models via Coordinate Descent". en. In: Journal of Statistical Software 33.1 (2010). DOI: $10.18637 /$ jss.v033.i01.

[112] M. Bertamini, M. Zito, N. E. Scott-Samuel, and J. Hulleman. "Spatial clustering and its effect on perceived clustering, numerosity, and dispersion". en. In: Attention, Perception, 6 Psychophysics 78.5 (July 2016), pp. 1460-1471. DOI: 10.3758/s13414-016-1100-0.

[113] N. Macquaide, H.-T. M. Tuan, J.-i. Hotta, W. Sempels, I. Lenaerts, P. Holemans, J. Hofkens, M. S. Jafri, R. Willems, and K. R. Sipido. "Ryanodine receptor cluster fragmentation and redistribution in persistent atrial fibrillation enhance calcium release". en. In: Cardiovascular Research 108.3 (Dec. 2015), pp. 387-398. DOI: $10.1093 / \mathrm{cvr} / \mathrm{cvv} 231$.

[114] T. Takamatsu and W. G. Wier. "Calcium waves in mammalian heart: quantification of origin, magnitude, waveform, and velocity." In: The FASEB Journal 4.5 (Mar. 1990), pp. 1519-1525. DOI: 10.1096/fasebj.4.5.2307330.

[115] A. Hatano, J.-i. Okada, T. Washio, T. Hisada, and S. Sugiura. "Distinct Functional Roles of Cardiac Mitochondrial Subpopulations Revealed by a 3D Simulation Model". en. In: Biophysical Journal 108.11 (June 2015), pp. 2732-2739. DOI: $10.1016 /$ j.bpj.2015.04.031. 
[116] H. Sutanto, B. van Sloun, P. Schönleitner, M. A. M. J. van Zandvoort, G. Antoons, and J. Heijman. "The Subcellular Distribution of Ryanodine Receptors and L-Type Ca2+ Channels Modulates Ca2+-Transient Properties and Spontaneous Ca2+-Release Events in Atrial Cardiomyocytes". English. In: Frontiers in Physiology 9 (2018). DOI: 10.3389/fphys.2018.01108.

[117] M. Fernandez-Velasco, A. Rueda, N. Rizzi, J.-P. Benitah, B. Colombi, C. Napolitano, S. G. Priori, S. Richard, and A. M. Gomez. "Increased Ca2+ Sensitivity of the Ryanodine Receptor Mutant RyR2R4496C Underlies Catecholaminergic Polymorphic Ventricular Tachycardia". en. In: Circulation Research 104.2 (Jan. 2009), pp. 201-209. DOI: 10.1161/CIRCRESAHA.108.177493.

[118] L. T. Izu, Y. Xie, D. Sato, T. Bányász, and Y. Chen-Izu. "Ca2+ waves in the heart". en. In: Journal of Molecular and Cellular Cardiology 58 (May 2013), pp. 118-124. DOI: 10.1016/j.yjmcc.2012.11.014.

[119] "Principal Components in Regression Analysis". en. In: Principal Component Analysis. Ed. by I. T. Jolliffe. Springer Series in Statistics. New York, NY: Springer, 2002, pp. 167-198. DOI: 10.1007/0-387-22440-8_8.

[120] B. Louis. "Multidimensional binary search trees used for associative searching". EN. In: Communications of the ACM (Sept. 1975). DOI: 10.1145/361002.361007.

[121] K. Konakli and B. Sudret. "Polynomial meta-models with canonical low-rank approximations: Numerical insights and comparison to sparse polynomial chaos expansions". en. In: Journal of Computational Physics 321 (Sept. 2016), pp. 11441169. DOI: $10.1016 / j . j c p .2016 .06 .005$.

[122] K. Konakli and B. Sudret. "Reliability analysis of high-dimensional models using low-rank tensor approximations". en. In: Probabilistic Engineering Mechanics 46 (Oct. 2016), pp. 18-36. DOI: 10.1016/j.probengmech.2016.08.002.

[123] J. I. Goldhaber, S. T. Lamp, D. O. Walter, A. Garfinkel, G. H. Fukumoto, and J. N. Weiss. "Local regulation of the threshold for calcium sparks in rat ventricular myocytes: role of sodium-calcium exchange". en. In: The Journal of Physiology 520.2 (Oct. 1999), pp. 431-438. DOI: 10.1111/j.1469-7793.1999. 00431.x. 



\section{Appendix A}

\section{Appendix}

\section{Variables, Parameters and Biomarkers of the Model}

Here the tables that contain the values of parameters and variables used in the mathematical model as well as the biomarkers employed in the study are listed. In the following a short summary of the tables is listed. Note: the parameters selected in this study, i.e. $k_{\text {plus }}, k_{\text {close }}, g_{\text {RyR }}, g_{\mathrm{LCC}}$ and $V p_{\max }$ have no fixed value.

- Table A.1 contains the literature values of the biomarkers used in the present study together with a short description and a reference.

- In tables A.2 and A.5 the main values that describe the dyadic cleft and the calcium diffusion in it are collected.

- Tables A.3 and A.9 contain the values of parameters describing all ionic currents and channels that are not related to LCCs or RyRs. Specifically the former table lists the parameters of NCX and SERCA pump too.

- Table A.4 contains parameter values involved in the opening and closing of LCCs as well as their permeability.

- In table A.6 the parameter values for the jSR calcium dynamics are listed.

- Table A.7 contains parameter values for the description of the diffusion dynamics of calcium ions and buffers in the cytoplasm and the SR as well as their volume properties. Table A.8 lists the initial values for calcium and buffer concentrations. 


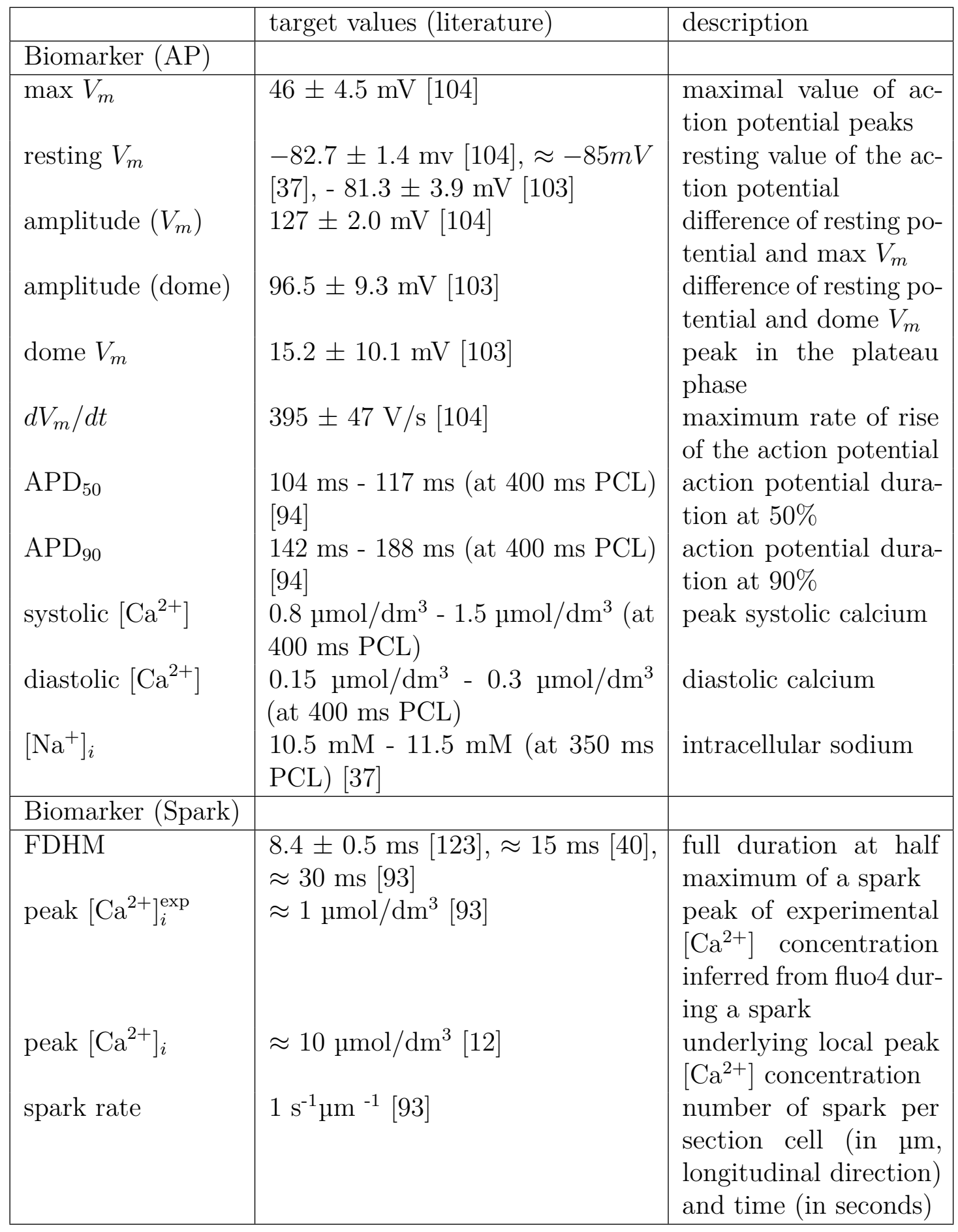

Table A.1 - Literature biomarker ranges for $\left[\mathrm{Ca}^{2+}\right]$ transients and action potentials. Note that for the values from [104], the standard deviation was calculated from the standard error by $\sigma=\sqrt{n} \hat{\sigma}$. See also figure 3.1. 


\begin{tabular}{|c|c|c|}
\hline Parameter & Meaning & Value \\
\hline$N_{\mathrm{RyR}}$ & distribution parameter for & 20 \\
\hline$N_{\mathrm{LCC}}$ & $\begin{array}{l}\text { average number of LCCs per } \\
\text { dyadic cleft }\end{array}$ & 4 \\
\hline$r_{\mathrm{RyR}, \mathrm{LCC}}$ & ratio of RyRs and LCCs & 5 \\
\hline$k_{\text {plus }}$ & $\begin{array}{l}\text { rate constant determining } \\
\text { the RyR opening rate }\end{array}$ & varies \\
\hline$k_{\text {close }}$ & closing rate (RyRs) & varies \\
\hline$\phi_{k}$ & $\begin{array}{l}{\left[\mathrm{Ca}^{2+}\right]_{j s r^{-}} \text {dependent regula- }} \\
\text { tion affinity }\end{array}$ & $1.59 \mathrm{mM}$ \\
\hline$\phi_{b}$ & $\begin{array}{l}{\left[\mathrm{Ca}^{2+}\right]_{j s r^{-}} \text {dependent regula- }} \\
\text { tion minimum }\end{array}$ & 0.8025 \\
\hline$\eta$ & $\begin{array}{l}c_{\mathrm{di}} \text { sensitivity Hill Coeffi- } \\
\text { cient }\end{array}$ & 2.1 \\
\hline$\mu_{\mathrm{RyR}}$ & $\begin{array}{l}\text { step length distribution pa- } \\
\text { rameter for average of RyR } \\
\text { placement }\end{array}$ & $40.1 \mathrm{~nm}$ \\
\hline$\sigma_{\mathrm{RyR}}$ & $\begin{array}{l}\text { step length distribution pa- } \\
\text { rameter for standard devia- } \\
\text { tion of RyR placement }\end{array}$ & $7.4 \mathrm{~nm}$ \\
\hline$r_{\min }$ & $\begin{array}{l}\text { minimum distance for place- } \\
\text { ment }\end{array}$ & $30 \mathrm{~nm}$ \\
\hline$r_{\mathrm{chb}}$ & $\begin{array}{l}\text { minimum RyR distance to } \\
\text { cleft boundary }\end{array}$ & $60 \mathrm{~nm}$ \\
\hline $\mathrm{g}_{R y R}$ & RyR permeability & varies \\
\hline
\end{tabular}

Table A.2 - Dyadic cleft parameters. 


\begin{tabular}{|c|c|c|}
\hline Parameter & Meaning & Value \\
\hline $\mathrm{g}_{\mathrm{NaCa}}$ & $\begin{array}{l}\begin{array}{l}\text { strength of } \\
\text { exchanger }\end{array} \\
\mathrm{Na}^{+} / \mathrm{Ca}^{2+}-\end{array}$ & $1.8 \mu \mathrm{mol} / \mathrm{dm}^{3} \mathrm{~s}^{-1}$ \\
\hline $\mathrm{f}_{\mathrm{NaCa} \text {,high }}$ & $\begin{array}{l}\text { maximal factor for } g_{\mathrm{NaCa}} \text { at } \\
\text { dyadic cleft centers }\end{array}$ & 72.0 \\
\hline $\mathrm{f}_{\mathrm{NaCa}, \text { low }}$ & $\begin{array}{l}\text { minimal factor for } g_{\mathrm{NaCa}} \text { dis- } \\
\text { tant to dyadic clefts }\end{array}$ & 0.66 \\
\hline $\mathrm{f}_{\mathrm{NaCa}, \text { surf }}$ & $\begin{array}{l}\text { minimal factor for } g_{\mathrm{NaCa}} \text { at } \\
\text { cell surface }\end{array}$ & 0.5 \\
\hline$V_{\mathrm{P}, \max }$ & $\begin{array}{l}\text { maximal rate of SERCA up- } \\
\text { take }\end{array}$ & varies \\
\hline$K_{\mathrm{P}}$ & SERCA uptake threshold & $0.4 \mu \mathrm{mol} / \mathrm{dm}^{3}$ \\
\hline$k_{\text {sat }}$ & constant & 0.2 \\
\hline$\xi$ & constant & 0.35 \\
\hline$K_{\mathrm{m}, \mathrm{Nai}}$ & constant & $12.3 \mathrm{mM}$ \\
\hline$K_{\mathrm{m}, \mathrm{Na} 0}$ & constant & $87.5 \mathrm{mM}$ \\
\hline$K_{\mathrm{m}, \mathrm{Cai}}$ & constant & $3.6 \times 10^{-3}$ \\
\hline$K_{\mathrm{m}, \mathrm{Cao}}$ & constant & $1.3 \mathrm{mM}$ \\
\hline$c_{\text {naca }}$ & constant & $0.3 \mu \mathrm{mol} / \mathrm{dm}^{3}$ \\
\hline
\end{tabular}

Table A.3 - Exchanger and uptake parameters.

\begin{tabular}{|c|c|c|}
\hline Parameter & Meaning & Value \\
\hline$s_{1}^{\prime}$ & voltage-inactivation rate & $0.00195 \mathrm{~ms}^{-1}$ \\
\hline$r_{2}$ & closing rate & $3.0 \mathrm{~ms}^{-1}$ \\
\hline$k_{p}^{0}$ & $\begin{array}{l}\text { half-rate }\left[\mathrm{Ca}^{2+}\right] \text { binding con- } \\
\text { stant }\end{array}$ & $180.0 \mathrm{\mu mol} / \mathrm{dm}^{3}$ \\
\hline$\tau_{p o}$ & average closed time & $1.0 \mathrm{~ms}$ \\
\hline$r_{1}$ & uninhibited open rate & $0.3 \mathrm{~ms}$ \\
\hline$k_{1}^{\prime}$ & voltage-inhibition rate & $0.00413 \mathrm{~ms}^{-1}$ \\
\hline$T_{\mathrm{Ba}}$ & fitting parameter & $450.0 \mathrm{~ms}$ \\
\hline$k_{2}$ & $\begin{array}{l}{\left[\mathrm{Ca}^{2+}\right]} \\
(\mathrm{LCCs})\end{array} \quad$ unbinding $\quad$ rate & $1.03615 \times 10^{-4} \mathrm{~ms}^{-1}$ \\
\hline$k_{2}^{\prime}$ & $\begin{array}{l}\text { voltage-Inhibition resolution } \\
\text { rate }\end{array}$ & $2.24 \times 10^{-4} \mathrm{~ms}^{-1}$ \\
\hline $\bar{c}_{p}$ & $\begin{array}{l}\text { half-rate constant for deep } \\
{\left[\mathrm{Ca}^{2+}\right] \text { inhibition }}\end{array}$ & $60.0 \mathrm{\mu mol} / \mathrm{dm}^{3}$ \\
\hline $\mathrm{g}_{L C C}$ & LCC permeability & varies \\
\hline
\end{tabular}

Table A.4 - Constants involved in computing transition rates for LCC channels. 


\begin{tabular}{|l|l|r|}
\hline Parameter & Meaning & Value \\
\hline$\phi_{0}$ & dimensionless constant & -2.2 \\
$\kappa$ & inverse of the Debye length \\
$D_{c}$ & $\begin{array}{l}\text { diffusion constant for } \\
{\left[\mathrm{Ca}^{2+}\right] \text { in the dyadic space }} \\
\text { height of the dyadic space }\end{array}$ & $0.1 \mathrm{~nm}^{2} / \mathrm{ms}^{-1}$ \\
$h$ & $\begin{array}{l}\text { cylinder } \\
\text { radius of the dyadic space }\end{array}$ & $15 \mathrm{~nm}$ \\
$R$ & cylinder & varies \\
& universal gas constant & $8.31 \mathrm{~kJ}(\mathrm{~K} \mathrm{~mol})^{-1}$ \\
$\mathrm{R}_{B}$ & temperature of the cell & $308 \mathrm{~K}$ \\
$\mathrm{~T}$ & buffering factor & 2 \\
$\beta_{d}$ & & \\
\hline
\end{tabular}

Table A.5 - Constants relevant to model diffusion inside the dyadic space.

\begin{tabular}{|l|r|r|}
\hline Parameter & Meaning & Value \\
\hline$B_{\text {csqn }}$ & total calsequestrin concentration & $800 \mu \mathrm{mol} / \mathrm{dm}^{3}$ \\
$n$ & number of calsequestrin binding sites & 15 \\
$K_{\text {csqn }}$ & dissociation constant of calsequestrin & $600 \mu \mathrm{mol} / \mathrm{dm}^{3}$ \\
$\nu_{\mathrm{jSR}}$ & volume of the jSR & varies \\
$\tau_{\text {refill }}$ & refill flux time constant & $0.5 \mathrm{~ms}$ \\
\hline
\end{tabular}

Table A.6 - Constants and parameters involved in modelling the $\left[\mathrm{Ca}^{2+}\right]$ dynamics in the jSR. 


\begin{tabular}{|c|c|c|}
\hline Parameter & Description & Value \\
\hline $\mathrm{b}_{m}^{t o t}$ & $\begin{array}{l}\text { total concentration of calmodulin } \\
\text { (mobile buffer) }\end{array}$ & $25.0 \mu \mathrm{mol} / \mathrm{dm}^{3}$ \\
\hline $\mathrm{b}_{s}^{\text {tot }}$ & $\begin{array}{l}\text { total concentration of troponin } \mathrm{C} \\
\text { (stationary buffer) }\end{array}$ & $70.0 \mathrm{\mu mol} / \mathrm{dm}^{3}$ \\
\hline $\mathrm{B}_{s r}^{t o t}$ & total concentration of jSR buffer & $1500.0 \mu \mathrm{mol} / \mathrm{dm}^{3}$ \\
\hline $\mathrm{b}_{\text {Fluo-4 }}^{\text {tot }}$ & total concentration of Fluo- 4 & $25.0 \mu \mathrm{mol} / \mathrm{dm}^{3}$ \\
\hline$D_{c}$ & diffusion constant of cytosolic $\left[\mathrm{Ca}^{2+}\right]$ & $0.22 \mu^{2} / \mathrm{ms}$ \\
\hline$D_{b_{m}}$ & diffusion constant of calmodulin & $0.04 \mathrm{\mu m}^{2} / \mathrm{ms}$ \\
\hline$D_{b_{s}}$ & diffusion constant of troponin $\mathrm{C}$ & $0 \mu \mathrm{m}^{2} / \mathrm{ms}$ \\
\hline$D_{b_{f}}$ & diffusion constant of Fluo-4 & $0.033 \mathrm{\mu m}^{2} / \mathrm{ms}$ \\
\hline$D_{S}$ & diffusion constant of sarcoplasmic $\left[\mathrm{Ca}^{2+}\right]$ & $0.2{\mu \mathrm{m}^{2}}^{2} / \mathrm{ms}$ \\
\hline $\mathrm{k}_{s}^{+}$ & on rate for troponin $\mathrm{C}$ binding & $0.043 \mathrm{\mu mol}^{-1} \mathrm{dm}^{-3} \mathrm{~ms}^{-1}$ \\
\hline $\mathrm{k}_{s}^{-}$ & off rate for troponin $\mathrm{C}$ binding & $0.026 \mathrm{~ms}^{-1}$ \\
\hline $\mathrm{k}_{m}^{+}$ & on rate for calmodulin binding & $0.023 \mathrm{\mu mol}^{-1} \mathrm{dm}^{-3} \mathrm{~ms}^{-1}$ \\
\hline $\mathrm{k}_{m}^{-}$ & off rate for calmodulin binding & $0.238 \mathrm{~ms}^{-1}$ \\
\hline$\nu_{s r} / \nu_{\text {cell }}$ & ratio of SR to cell volume & 0.08 \\
\hline$\nu_{j s r} / \nu_{c e l l}$ & ratio of jSR to cell volume & 0.005 \\
\hline$\nu_{c y t} / \nu_{\text {cell }}$ & ratio of cytosolic volume to cell volume & 0.915 \\
\hline
\end{tabular}

Table A.7 - Buffering and diffusion parameters.

\begin{tabular}{|l|c|r|}
\hline Parameter & Description & Value \\
\hline$c$ & free cytoplasmic $\left[\mathrm{Ca}^{2+}\right]$ concentration & $0.1 \mathrm{\mu mol} / \mathrm{dm}^{3}$ \\
$b_{x}$ & buffered cytosolic $\left[\mathrm{Ca}^{2+}\right]($ for $x=m, s, f)$ & $\frac{b_{x}^{t o t} \cdot c}{k_{x}^{+} / k_{x}^{-}+c}$ \\
$S$ & free sarcoplasmic $\left[\mathrm{Ca}^{2+}\right]$ concentration & $1200 \mu \mathrm{mol} / \mathrm{dm}^{3}$ \\
\hline
\end{tabular}

Table A.8 - Initial Values.

\begin{tabular}{|l|l|r|}
\hline Parameter & Meaning & Value \\
\hline $\mathrm{g}_{\mathrm{Na}}$ & peak $\mathrm{I}_{\mathrm{Na}}$ conductance & $12.0 \mathrm{mS} / \mu \mathrm{m} \mathrm{F}$ \\
$\mathrm{g}_{\mathrm{to}, \mathrm{f}}$ & peak $\mathrm{I}_{\mathrm{to}, \mathrm{f}}$ conductance & $0.11 \mathrm{mS} / \mu \mathrm{m} \mathrm{F}$ \\
$\mathrm{g}_{\mathrm{to}, \mathrm{s}}$ & peak $\mathrm{I}_{\mathrm{to}, \mathrm{s}}$ conductance & $0.04 \mathrm{mS} / \mu \mathrm{m} \mathrm{F}$ \\
$\mathrm{g}_{\mathrm{K} 1}$ & peak $\mathrm{I}_{\mathrm{K} 1}$ conductance & $0.3 \mathrm{mS} / \mu \mathrm{m} \mathrm{F}$ \\
$\mathrm{g}_{\mathrm{Kr}}$ & peak $\mathrm{I}_{\mathrm{Kr}}$ conductance & $0.0125 \mathrm{mS} / \mu \mathrm{m} \mathrm{F}$ \\
$\mathrm{g}_{\mathrm{Ks}}$ & peak $\mathrm{I}_{\mathrm{Ks}}$ conductance & $0.1386 \mathrm{mS} / \mu \mathrm{m} \mathrm{F}$ \\
$\mathrm{g}_{\mathrm{NaK}}$ & peak $\mathrm{I}_{\mathrm{Kr}}$ conductance & $1.5 \mathrm{mS} / \mu \mathrm{m} \mathrm{F}$ \\
\hline
\end{tabular}

Table A.9 - Ionic current conductances. 


\section{Acknowledgements}

Conducting research and writing a doctoral thesis would not have been possible for me without the support of many people. I cannot mention every single person or colleague, still I would like to thank those who assisted the most.

First of all, I would like to thank Ulrich Parlitz and Stefan Luther, who gave me the opportunity to do a $\mathrm{PhD}$ in the Research Group Biomedical Physics. The atmosphere in the group constantly pushed me towards new insights and represented the perfect soil in which I could let my curiosity for the world grow freely. Further, being introduced to the field of cardiac research was a great enrichment for me.

I would like to thank my office colleague Florian Spreckelsen, with whom I shared plenty fascinating discussions that ranged from research topics to climate politics. We also met in a weekly one-to-one journal club that deepened my literature knowledge on our research topics tremendously. Finally I would like to thank him for carefully proofreading my thesis and for being a "Leidens- und Freudengenosse" throughout this journey.

Furthermore, I would like to thank Martin Falcke who gave me the opportunity to use the mathematical model I employed in this project and to experience a collaboration with a group in a different city. This collaboration had a third main actor: Wolfgang Giese. With him I enjoyed fruitful discussions and shared ideas about the research on cardiac muscle cells. I would like to thank him for introducing me to the field of uncertainty quantification that surrounds the cardiac mathematical modelling.

A big thank-you goes to all the colleagues with whom I shared not only on office but also many laughs and exchanged valuable ideas. Baltasar Rüchardt was always available for some good distraction or a thoughtful chat. Edda Boccia was a moral support in the beginning of my $\mathrm{PhD}$, always showing positivity. Alexander Schlemmer, Henrik tom Wörden, Sebastian Berg, Daniel Hornung and Tariq Baig enriched my day-to-day life with inspiring and vanguard software ideas and with long-lasting and distracting board game evenings. My Bachelor student Frederik Böddeker, whom I mentored for several months, indirectly taught me some supervision basics, thank you for that. I would also like to mention Jan Christoph, Thomas Lilienkamp, Annette Witt, Oskar Schnaack, Johannes Schöder-Schetelig, Jan Lebert, Sayadeh Hussaini, Raúl Quiñonez and Vineesh Kappadan as kind and valuable colleagues.

Many thanks go to Isabella Guido and Laura Turco, who, together with Edda, created a sort of "Little Italy". Especially with Isabella I could discuss with pleasure cooking interests and Italian politics.

Jana Lasser and Felix Bäuerle I would like thank for the many years spent in the 
effort to improve the working conditions of the PhDs. In this regard, I also want to thank all the people I had the pleasure of meeting and working with at the Max Planck's PhDnet.

"Man braucht immer so viel Zeit, wie man hat". Ulrich Parlitz supervised my work excellently. I could not wish to have had more freedom in directing my interests and, at the same time, to have felt safe when important questions came up. His valuable scientific and personal advice allowed me to grow immensely.

I would like to thank my thesis advisory committee members Stefan Klumpp and Andreas Neef for challenging questions, important suggestions and great scientific input. I would like to thank the further member of my examination board, David Zwicker, too.

I would like to thank Antje Erdmann, Frauke Bergmann and the whole Göttingen Graduate Center for Neurosciences, Biophysics, and Molecular Biosciences (GGNB) for the great organisation and help that this graduate school provides for conducting research as a $\mathrm{PhD}$.

Vielen Dank an Katharina Krischer, die mich auf den spannenden Pfad der nichtlinearen Dynamik gesetzt hat und die mich an die Grundlagen wissenschaftlichen Denkens zuerst herangeführt hat.

Drei guten Freunden in München will ich ebenfalls danken: Johannes Heinbuch für die vielen bereichenden Diskussionen, die mir stets andere Blickpunkte gezeigt haben. Mit David Egger habe ich seit Studienanfang alle Phasen unseres wissenschaftlichen Werdegangs wunderbar erlebt; das hat auf der Distanz auch nicht aufgehört. Felix Kemeth hat meine Neugier auf immer unterschiedliche Art und Weise in unzähligen Gesprächen positiv angeregt.

An dieser Stelle will ich auch meinem Ultimate Team, die "Göttinger 7", für den wunderbaren Ausgleich durch Training und Turnieren danken.

Un pensiero va ad Alessandro Gentili e Stefano Caccialupi, che hanno colmato innumerevoli serate con spassose diversioni online creando un bellissimo e periodico contatto. Niccoló Bitossi mi ha accompagnato con intense discussioni e ritrovi che mi hanno sempre ricordato di quanto fosse grande il mondo al di fuori dalla mia realtà, grazie per questo. Grazie anche a Francesco Sacco per un'amicizia profonda, che mi ha sempre regalato sicurezza e sana follia.

Vielen Dank an meine Mutter Anna Thiele, die mich mit ihrer Präsenz motiviert, die mich oft so gut versteht und die mein Studium in Deutschland ermöglich hat. Grazie anche a mio padre Francesco Cosi per avermi avvicinato al software libero fin da giovane e a mia sorella Lisa Cosi per le tante diversioni culinarie che abbiamo condiviso. Vielen Dank an Selene Mariani für die unzähligen wunderschönen, ironischen und aufbauenden Momente, die mich immer repariert haben und die eine wunderbare und liebevolle Realität darstellen. 
